Analytic Scalings of the

Constant-Neutralization Beam Envelope Equation, with Applications

J. F. McCarrick

November 5, 2007 
This document was prepared as an account of work sponsored by an agency of the United States government. Neither the United States government nor Lawrence Livermore National Security, LLC, nor any of their employees makes any warranty, expressed or implied, or assumes any legal liability or responsibility for the accuracy, completeness, or usefulness of any information, apparatus, product, or process disclosed, or represents that its use would not infringe privately owned rights. Reference herein to any specific commercial product, process, or service by trade name, trademark, manufacturer, or otherwise does not necessarily constitute or imply its endorsement, recommendation, or favoring by the United States government or Lawrence Livermore National Security, LLC. The views and opinions of authors expressed herein do not necessarily state or reflect those of the United States government or Lawrence Livermore National Security, LLC, and shall not be used for advertising or product endorsement purposes.

This work performed under the auspices of the U.S. Department of Energy by Lawrence Livermore National Laboratory under Contract DE-AC52-07NA27344. 


\section{Analytic Scalings of the Constant-Neutralization Beam Envelope Equation, with Applications}

J. McCarrick
March 3, 2005

$\underline{\text { Table of Contents }}$

1. Introduction _ 2

2. Conceptual Background__ 3

3. Basic Assumptions__ 6

4. Two Special Cases___ 8

5. General Case ( $\varepsilon$, K Non-Zero)__ 12

6. Validity in the Presence of a Final Focus Magnet___ 31

7. Implications for Spot Control__ 34

8. Summary of Major Results___ 46

9. References 47

Appendix A. Algebraic Details of the Analytic Solutions__ 48 


\section{$\underline{\text { 1. Introduction }}$}

Neutralized transport of relativistic electron beams can achieved in various circumstances. In one form, the beam is transported through a plasma, either pre-formed or beam generated, where the plasma electrons are ejected due to the space charge influence of the beam. The beam can be fully neutralized this way if the plasma is sufficiently dense. Typically, the transport physics of concern in this case are the various macro- and micro-instabilities that can develop due to interactions of the beam with the plasma; charge and current neutralization are certainly important but tend to be just one set of concerns among many. The study of beam/plasma interactions has been active for many years [e.g. 1].

In a different scenario, the beam impinges on a plasma with a sharp boundary (as maintained on the timescale of a beam pulse) and, via space charge, extracts ions from the plasma; extraction energies can be hundreds of kilovolts in the case of tightly focused, high current beams. In this case, the ions have a lower density than the beam and are not accompanied by a plasma electron population; the main transport issue is charge neutralization. Such a sharply bounded plasma can occur via ionization of surface impurities from a solid target; the transport of the beam through this thin layer is typically not of interest relative to the transport upstream of the surface and the beam/target interactions beyond the surface. Since the partial neutralization of the beam changes its focusing characteristics on the target, and since the high extraction energy means the ion column is moving rapidly into the beam and introducing strong time variation, this "backstreaming ion" phenomenon has been an area of active study in the transport of the high-intensity electron beams used in radiographic accelerators (see [2] for an example of such machines). However, much of the work has been experimental [3] and numerical [4]. The conceptual understanding provided by pencil-and-paper analysis thus far $[5,6]$ has covered the important topics (such as disruption length and neutralization fraction, to be defined later) but generally in the context of idealized beam envelopes that lack enough detail to examine the "control knobs" in a focusing system, such as focal length, time-varying magnetic lenses, or time-averaged focusing.

The obvious reason for the lack of analytic results is that they are hard to come by, even in highly simplified scenarios. This study will make quite a few simplifying assumptions and still will not produce much in the way of simple closed-form answers. However, it is possible to provide limiting expressions for some of the quantities of interest, and for others there are simple algebraic systems that can studied via spreadsheet rather than a more time-consuming analysis package. Furthermore, the reduced expressions also provide a fast model that can be embedded in more complex parametric or optimization studies, where repeated calls to something like a PIC numerical model would be impractical. 


\section{Conceptual Background}

The conceptual picture of the effect of backstreaming ions on electron beams has been presented in many of the references, but is repeated here for completeness. Consider a relativistic electron beam which is being brought to a focus at a particular plane perpendicular to the axis of beam propagation:

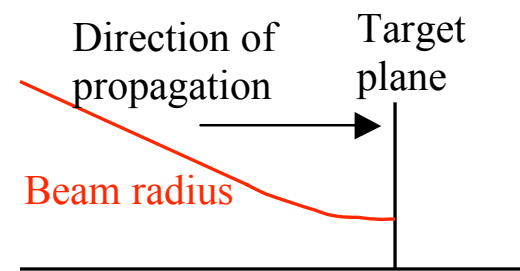

Figure 2.1. Focusing of an electron beam on a target plane visualized using an average beam radius versus position.

The beam radius as sketched above is some sort of average representation of the beam's radial distribution, which in reality is neither uniform nor sharply bounded in space. In this report we consider the root-mean-squared (RMS) value, which is not easy to measure but which lends itself to analysis techniques that have rigorous justification.

For relativistic beams, the electric field due to the beam's own space charge and the magnetic field due to its current produce forces on the beam that cancel to order $1 / \gamma^{2}$, where $\gamma$ is the relativistic gamma. Near a tight focus, the beam transport is dominated by the spread in transverse velocities, characterized not by a temperature (since the distribution in phase space is often not thermal) but a quantity called emittance, which is related to the "area" of the beam's transverse phase space. In this report, we will work exclusively with the unnormalized RMS or "Lee-Cooper" emittance [7].

When we add ions into the beam, the delicate balance between the forces due to space charge and space current is upset; even when the ion density is the same as the beam density, the ion velocity is much less than $c$ and so the ion current is typically negligible. The result is that the beam volume contains less net charge than when unperturbed but still carries the same current; the magnetic forces win out and the beam pinches down relative to its original configuration. If we add a column of ions extending out from the original focal plane in figure 2.1, we might expect to see something like figure 2.2:

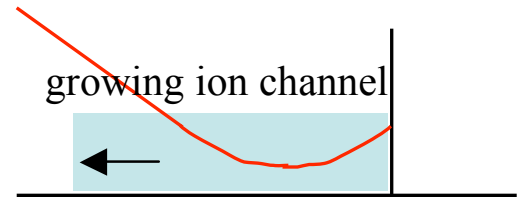

Figure 2.2. Pinching of the beam envelope due to the addition of ion space charge forces. 
The surface on which the beam is impinging is typically grounded (if it isn't, the strong radial electric field from the beam will typically cause the target to break down and discharge to the nearest surface that is grounded). Far from the target, the center of the beam is at considerable negative potential relative to grounded pipe in which it travels; this is considered in many beam physics texts [e.g. 8] and so the value here will be given without proof,

$$
\phi(r=0)=30 I(1+2 \ln b / a)
$$

where $\mathrm{I}$ is the beam current in amps, $\mathrm{b}$ is the radius of the pipe, $\mathrm{a}$ is the radius of the beam, and $\phi$ is the potential in volts.

A beam focused to a millimeter-sized spot in a beam pipe of a few centimeter radius will have a potential drop of hundreds of kilovolts. For the axial structure of this potential near a grounded target, consider that the potential must vary from zero at the target to the full beam potential far from the target, and the only scale length available is the beam radius. So within a few millimeters, the ions have been accelerated to an energy of order of the beam potential (it will vary somewhat with radius); for a light ion such as $\mathrm{H}+$, this corresponds to a high velocity, $\sim 0.02 c$. During a beam pulse of tens of nanoseconds (typical for radiographic systems), the ions can move tens of centimeters, introducing a rapid time variation in the beam transport. Schematic snapshots of the envelope with time, and the size of the beam at the original focal plane, are shown in figure 2.3.

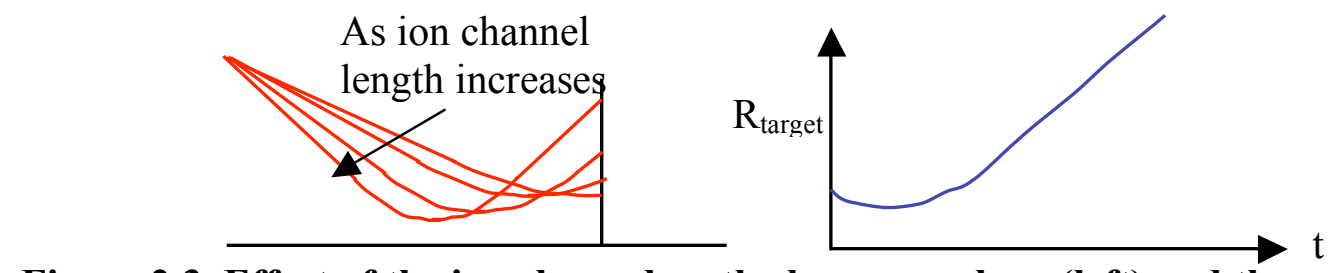

Figure 2.3. Effect of the ion channel on the beam envelope (left) and the spot size on the target plane (right) as the channel grows with time.

When this effect is undesirable (which is certainly the case in radiographic systems, which depend on maintaining a small focus size for high resolution), there are certain quantities of particular interest. The first is the disruption length, defined as the length of an ion column, at fixed neutralization, through which the beam will pinch down and then return to its original focal size on the original focal plane. This is illustrated in figure 2.4.

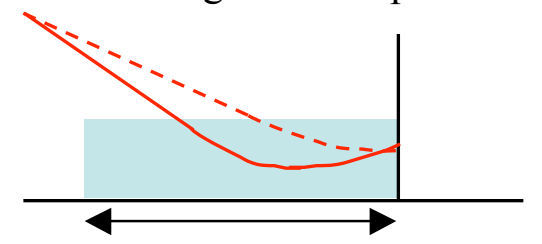

Disruption length

Figure 2.4. Illustration of the disruption length, as defined by the channel length at which the perturbed beam size on target returns to its original size. 
The second quantity of interest is the disruption rate, which is the rate of spot size growth on the original target plane as the ion column grows beyond a disruption length. It corresponds to the slope of the right-hand curve in figure 2.3. 


\section{$\underline{\text { 3. Basic Assumptions }}$}

The starting point for this analysis consists of a set of assumptions whose aim is to allow the use of the Lee-Cooper RMS envelope equation [7] in the simplest possible form:

1. The beam is axially symmetric

2. The particle motion is paraxial

3. The energy is constant

4. $\beta \sim 1$

5. The emittance is constant, even when ions are present

6. The ion density can be expressed as a fraction of the beam density (the neutralization fraction), and this fraction is independent of radius or axial location

7. The beam was born in zero magnetic field

8. Transport near the focus has no external fields

9. Transport near the focus is emittance-dominated when no ions are present

For the induction linacs used in radiography (which set the context for this study), assumptions (1), (3), (4), (7), and (9) are satisfied. For the higher energy machines such as the two axes of the DARHT facility, (2) and (8) are also satisfied, but this is not the case at the ETA-II experimental facility, where a strong final focus magnet brings the beam to the target at a significant slope. Full PIC simulations show that (5) is violated but not in drastic fashion; the variation is typically monotonic over the channel length and within less than a factor of two of the incoming value. Assumption (6) is made purely for analytic convenience, although far from the ion emission surface the radially averaged neutralization does become independent of $\mathrm{z}$.

The end result is that the RMS envelope equation takes the simple form

$$
\frac{d^{2} R}{d z^{2}}=\frac{\left(1-\gamma^{2} f\right) I}{\gamma^{3} I_{A} R}+\frac{\epsilon^{2}}{R^{3}}
$$

where $\mathrm{z}$ is the axial coordinate $(\mathrm{m}), \mathrm{R}$ is the RMS radius $(\mathrm{m}), \mathrm{I}$ is the beam current $(\mathrm{A}), \mathrm{I}_{\mathrm{A}}$ is the Alven current for electrons $\sim 17000 \mathrm{~A}$, $\mathrm{f}$ is the neutralization fraction, $\gamma$ the relativistic gamma, and $\varepsilon$ the unnormalized RMS emittance (in meter-radians).

The explicit form of assumption (9) is that when $\mathrm{f}$ is non-zero, it is much greater than $1 / \gamma^{2}$, so that we may write

$$
\frac{d^{2} R}{d z^{2}}=\frac{-K}{R}+\frac{\epsilon^{2}}{R^{3}}
$$

where $\mathrm{K}$ is a positive number given approximately by $\mathrm{fI} / \gamma \mathrm{I}_{\mathrm{A}}$; when there is no neutralization, the space charge term is then dropped altogether. 
The emittance-dominated solution $(\mathrm{K}=0)$ is well known; for general initial conditions of $\mathrm{R}=\mathrm{R}_{\mathrm{i}}$ and $\mathrm{R}^{\prime}=\mathrm{R}^{\prime}{ }_{\mathrm{i}}$ at $\mathrm{z}=\mathrm{Z}_{\mathrm{i}}$, the solution is

$$
\begin{aligned}
& R^{2}=R_{\text {min }}^{2}+\left[\sqrt{R_{i}^{2}-R_{m i n}^{2}}+\frac{R_{i}^{\prime}}{\left|R_{i}^{\prime}\right|} \frac{\epsilon\left(z-z_{i}\right)}{R_{m i n}}\right]^{2} \\
& R_{\text {min }}^{2} \equiv \frac{R_{i}^{2}}{1+\frac{R_{i}^{2} R^{\prime 2}}{\epsilon^{2}}}
\end{aligned}
$$

The location where the beam takes its minimum value, $\mathrm{R}=\mathrm{R}_{\min }$, is

$$
z_{\text {min }}=z_{i}-\frac{R_{i}^{\prime}}{\left|R_{i}^{\prime}\right|} \frac{R_{m i n}}{\epsilon} \sqrt{R_{i}^{2}-R_{m i n}^{2}}
$$

When the beam starts at a waist $\left(\mathrm{R}^{\prime}=0\right)$, the solution has the particularly simple form

$$
R^{2}=R_{m i n}^{2}+\frac{\epsilon^{2}\left(z-z_{m i n}\right)^{2}}{R_{m i n}^{2}}
$$

Far from the waist location,

$$
R \approx \frac{\epsilon\left(z-z_{\min }\right)}{R_{\min }}, \quad R^{\prime} \approx \frac{\epsilon}{R_{\min }}
$$




\section{Two Special Cases}

Two particular cases of equation (3.1) have very simple analytic solutions. The first is the well-known case of matched transport in the ion-focused regime (IFR), which we will include here for completeness and to point out one interesting feature relative to the second special case.

Complete IFR physics also deals with any background neutral plasma (in the over-dense case) and instabilities related thereto. Here, however, we focus only on the macroscopic beam transport described by the envelope equation, in which case IFR corresponds to small perturbations about $\mathrm{R}=\mathrm{R}_{\mathrm{eq}}, \mathrm{R}^{\prime}=0$, where:

$$
R_{e q}^{2} \equiv \epsilon^{2} / K
$$

We will pick a phase point in $\mathrm{z}$ called $\mathrm{z}_{\mathrm{o}}$ such that $\mathrm{R}\left(\mathrm{z}_{\mathrm{o}}\right)=\mathrm{R}_{\mathrm{eq}}+\delta_{\mathrm{o}}, \mathrm{R}^{\prime}\left(\mathrm{z}_{\mathrm{o}}\right)=0$, where $\delta_{\mathrm{o}} / \mathrm{R}_{\mathrm{eq}}$ $<<1$. Substituting $R(z)=R_{e q}+\delta(z)$ into (3.1), carrying out the first-order Taylor expansion, and canceling the equilibrium terms gives

$$
\delta^{\prime \prime}=\left(\frac{K}{R_{e q}^{2}}-\frac{3 \epsilon^{2}}{R_{e q}^{4}}\right) \delta=-\frac{2 K}{R_{e q}^{2}} \delta
$$

whose solution is a simple sinusoid; the total solution for $\mathrm{R}$ is

$$
R(z)=R_{e q}+\delta_{o} \cos k\left(z-z_{o}\right), \quad k=\sqrt{2 K} / R_{e q}
$$

The disruption length is illustrated in Figure 4.1a; it corresponds to a single period of oscillation and is given by

$$
L_{D}=2 \pi / k=2 \pi R_{e q} / \sqrt{2 K}
$$

The second case to be considered here is the limit of a zero-emittance beam. As defined within the context of the assumptions in section III, this refers to the transport within the ion channel - the transport conditions outside the channel can be emittance-dominated and still make this case a realistic limit.

We take equation (3.1) and set $\varepsilon=0$ :

$$
R^{\prime \prime}=-K / R
$$

Multiplying this autonomous equation by R' and integrating once, we obtain

$$
R^{\prime 2}=R_{i}^{\prime 2}-2 K \ln R / R_{i}
$$

Choosing the sign convention for R' corresponding to a converging incoming beam, we convert this to a quadrature form: 


$$
\int_{R_{i}}^{R} \frac{d R}{\sqrt{-2 K \ln \frac{R}{R_{i} e^{R^{\prime 2} / 2 K}}}}=-\left(z-z_{i}\right)
$$

This integral can be made recognizable via two changes of variable. First,

$$
\begin{gathered}
x=\frac{R}{R_{\max }}, R_{\text {max }} \equiv R_{i} e^{R^{\prime 2} / 2 K} \\
\int_{R_{i} / R_{\max }}^{R / R_{\max }} \frac{d x}{\sqrt{-\ln x}}=-\sqrt{2 K} \frac{z-z_{i}}{R_{\max }}
\end{gathered}
$$

Then,

$$
\begin{aligned}
& y=\sqrt{-\ln x} \\
& \frac{2}{\sqrt{\pi}} \int_{\sqrt{-\ln R_{i} / R_{\max }}}^{\sqrt{-\ln R / R_{\max }}} e^{-y^{2}} d y=\sqrt{\frac{2 K}{\pi}} \frac{z-z_{i}}{R_{\max }}
\end{aligned}
$$

The integral is the error function, yielding a closed-form expression for $\mathrm{z}(\mathrm{R})$ :

$$
\operatorname{erf}\left(\sqrt{-\ln R / R_{\max }}\right)-\operatorname{erf}\left(\left|R_{i}^{\prime}\right| / \sqrt{2 K}\right)=\sqrt{\frac{2 K}{\pi}} \frac{z-z_{i}}{R_{\max }}
$$

Approximate inversions for $\mathrm{R}(\mathrm{z})$ tend to have limited scope. More interesting are the limiting expressions for the disruption length $L_{D}$. In terms of an unperturbed spot size $R_{S}$ at a fixed target plane located at $\mathrm{z}=\mathrm{Z}_{\mathrm{t}}$, we note that the size of the beam, $\mathrm{R}_{\mathrm{i}}$, entering the ion channel at $\mathrm{z}=\mathrm{Z}_{\mathrm{ch}}$, is

$$
R_{i}=R_{s}-R_{i}^{\prime}\left(z_{t}-z_{c h}\right)
$$

Let us relax the notation a bit by letting R' refer to the magnitude of the slope, i.e., a positive quantity even for a converging beam. The disruption length is found by calculating twice the distance spanned between the incoming radius $R_{i}$ and the point where the radius "bounces" through $\mathrm{R}=0$ (let us not dwell on maintaining zero emittance through such a bounce!), less the distance spanned between $R_{i}$ and $R_{s}$ following the bounce:

$\frac{L_{D}}{R_{s}+R^{\prime} L_{D}} e^{-R_{i}^{\prime 2} / 2 K} \sqrt{\frac{2 K}{\pi}}=2-\operatorname{erf}\left(R_{i}^{\prime} / \sqrt{2 K}\right)-\operatorname{erf}\left(\sqrt{R_{i}^{\prime 2} / 2 K+\ln \left(1+R_{i}^{\prime} L_{D} / R_{s}\right)}\right)$

If we define 


$$
x \equiv \sqrt{2 K} \frac{L_{D}}{R_{s}}, \quad \alpha \equiv \frac{R_{i}^{\prime}}{\sqrt{2 K}}
$$

and use the limiting expressions

$$
\begin{aligned}
& \operatorname{erf}(x<<1) \approx \frac{2 x}{\sqrt{\pi}} e^{-x^{2}} \\
& \operatorname{erf}(x>>1) \approx 1-\frac{e^{-x^{2}}}{x \sqrt{\pi}}\left(1-1 / 2 x^{2}\right)
\end{aligned}
$$

then we can calculate the disruption length in the small- and large-alpha limits:

$\alpha<<1$ :

$$
\begin{aligned}
& \frac{x}{1+\alpha x} e^{-\alpha^{2}} / \sqrt{\pi} \approx 2-\frac{2 \alpha e^{-\alpha^{2}}}{\sqrt{\pi}}-\frac{2 \sqrt{\alpha^{2}+\ln (1+\alpha x)}}{\sqrt{\pi}(1+\alpha x)} e^{-\alpha^{2}} \\
& x \approx(1+\alpha x)(2 \sqrt{\pi}-2 \alpha)-2 \sqrt{\alpha^{2}+\ln (1+\alpha x)} \\
& \approx \frac{2 \sqrt{\pi}-2 \alpha-2 \sqrt{\alpha^{2}+\ln (1+\alpha x)}}{1-2 \alpha \sqrt{\pi}} \\
& \approx 2 \sqrt{\pi}\left(1-\sqrt{\frac{2 \alpha}{\sqrt{\pi}}}\right) \\
& L_{D} \approx R_{s} \sqrt{\frac{2 \pi}{K}}\left(1-\sqrt{\frac{2 R_{i}^{\prime}}{\sqrt{2 \pi K}}}\right)
\end{aligned}
$$

$\alpha>>1$ :

$$
\begin{aligned}
\frac{1}{1+1 / \alpha x} \frac{e^{-\alpha^{2}}}{\alpha \sqrt{\pi}} & \approx \frac{e^{-\alpha^{2}}}{\alpha \sqrt{\pi}}\left(1-1 / 2 \alpha^{2}\right)+\frac{e^{-\alpha^{2}}}{\sqrt{\pi\left(\alpha^{2}+\ln (1+\alpha x)\right)}} \frac{1}{1+\alpha x} \\
1-1 / \alpha x & \approx 1-1 / 2 \alpha^{2}+\frac{1}{\alpha x}(1-1 / \alpha x)\left(1-\frac{\ln (1+\alpha x)}{2 \alpha^{2}}\right) \\
x & \approx 4 \alpha \\
L_{D} \approx \frac{2 R_{s} R_{i}^{\prime}}{K} &
\end{aligned}
$$

Considering the case where $\mathrm{R}^{\prime}=0$, we get

$$
L_{D}=R_{i} \sqrt{\frac{2 \pi}{K}}
$$


This is illustrated in figure $4.1 \mathrm{~b}$. For a hard-edged beam (where $R_{\text {edge }}=\sqrt{ } 2 R_{i, R M S}$ ) the above expression is the disruption length found through a combination of numerical experiment and insight in the paper by Caporaso [5], which is the one referred to as "the" disruption length when studying backstreaming ion amelioration techniques for radiographic accelerators [9]. It differs by $\pi^{1 / 2}$ relative to the perturbed IFR case (plus an adjustment of order $\delta_{\mathrm{o}} / \mathrm{R}_{\mathrm{eq}}$ which we ignore). Thus the point we wished to make by examining these two limits is that the disruption length must depend on incoming beam conditions and is not well-defined beyond a factor of two or so.

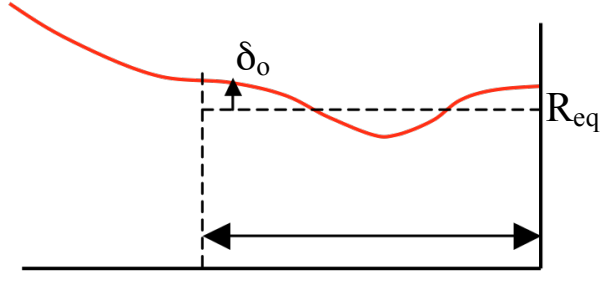

A: $L_{D}$, Case 1

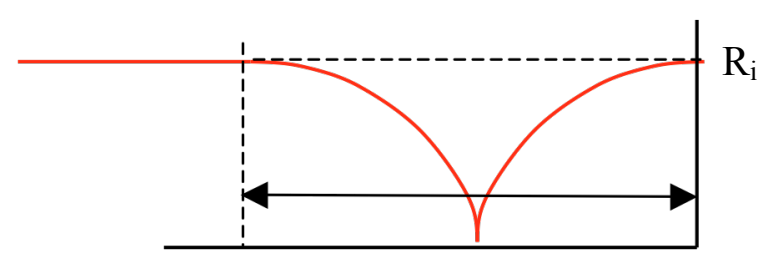

B: $L_{D}$, Case 2

Figure 4.1. The disruption lengths corresponding to two special cases. A) Small oscillations about equilibrium. B) Zero emittance and zero incoming slope. 


\section{General Case ( $\varepsilon$, K Non-Zero)}

In this section we consider the behavior of the full equation (3.1), retaining both emittance and ion focusing for arbitrary incoming beam conditions. It is useful to consider the general behavior of the beam under these conditions. Integration of (3.1) gives the following expression for the slope of the envelope, $\mathrm{dR} / \mathrm{dz}$ :

$$
R^{\prime 2}=R_{i}^{\prime 2}+1 / R_{i}^{2}-1 / R^{2}-\ln R^{2} / R_{i}^{2}
$$

This equation has two roots, locations where $\mathrm{dR} / \mathrm{dz}=0$; see Appendix A for a plot of the phase space. Repeated differentiation of (3.1) at locations where the slope is zero shows that all odd derivatives are zero; the envelope is symmetric about these points. For such symmetry to exist about two points, the solution must be periodic and the two zeroes of $\mathrm{dR} / \mathrm{dz}$ correspond to globally minimum and maximum values of $\mathrm{R}$, denoted $\mathrm{R}_{\min }$ and $\mathrm{R}_{\max }$, respectively. In between these radii, the envelope passes through an inflection point at:

$$
R=\frac{\epsilon}{\sqrt{K}} \equiv R_{e q}
$$

where $d^{2} R / d z^{2}=0$. Between $R_{\min }$ and $R_{\max }$ the envelope follows a half-cycle of a form of distorted sinusoid; the two "quarter-cycles" on either side of $\mathrm{R}_{\text {eq }}$ can have extremely different proportions due to the nonlinearity of the system. The incoming beam from a region upstream of the ion channel joins into some subsection of this sinusoid in a smooth manner. Figure 5.1 illustrates these general features.

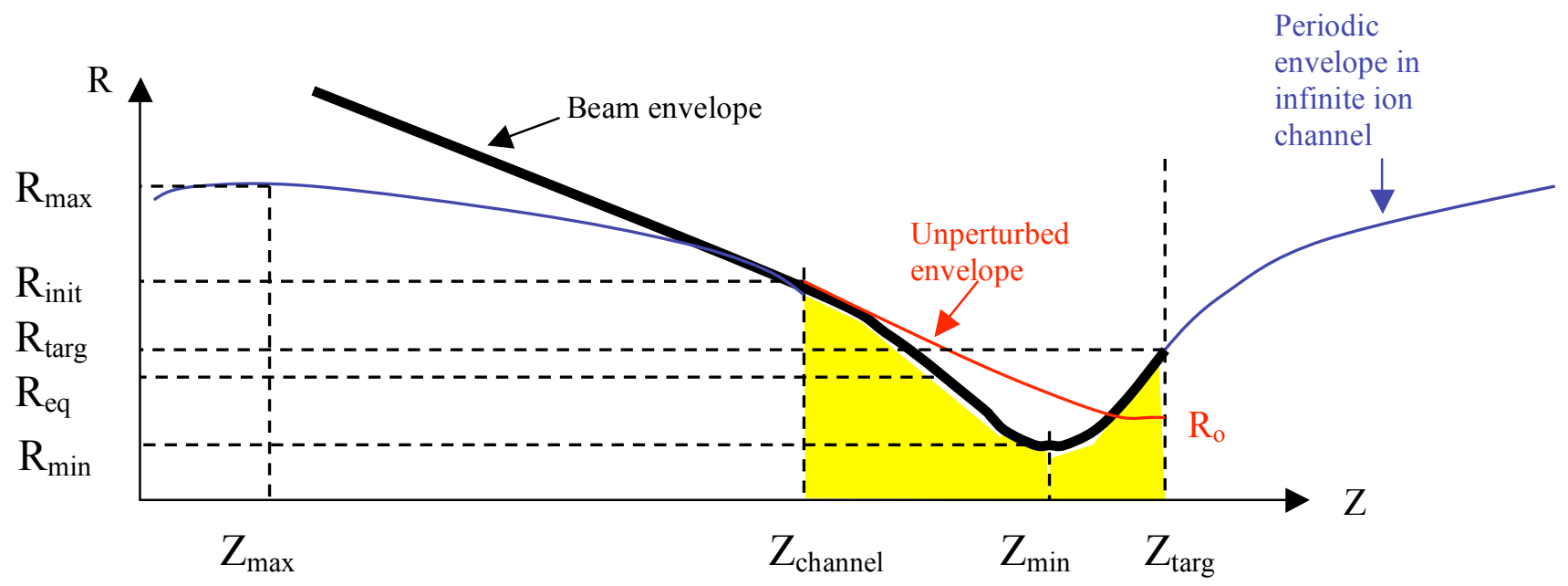

Figure 5.1. General features of beam envelope entering ion channel.

Note that depending on where the beam enters the ion channel and the location of the target plane, all of the reference values $\mathrm{R}_{\min }, \mathrm{R}_{\max }$, and $\mathrm{R}_{\text {eq }}$ may be "fictional" in the sense that the beam radius takes on none of these values within the physical channel. While the 
beam must enter the channel at a value less than or equal to $R_{\max }$, it can be larger than $\mathrm{R}_{\max }$ outside of it.

Let us begin a more quantitative analysis.

\subsection{Normalized Variables and Nominal Beams}

We will work in a normalized form by defining the new variables:

$$
\begin{aligned}
& p \equiv R / R_{e q} \\
& x \equiv z \sqrt{K} / R_{e q}
\end{aligned}
$$

where $\mathrm{R}_{\mathrm{eq}}$ is as defined previously. In these variables, (3.1) takes on a parameter-free form:

$$
p^{\prime \prime}=\frac{1}{p^{3}}-\frac{1}{p}
$$

Appendix A steps through a process for finding approximate solutions to this equation, by breaking the envelope into two regions, $p<1$ and $p>1$. There are a fair number of intermediate quantities required in order to implement the solution and it could be argued that the effort to use the solution as presented is greater than what it takes to solve the system numerically. However, the power of the analytic solution is in the ability to extract limiting behaviors and gain insight into the general behavior of the system.

While (5.1.1) does not have any explicit parameters, it remains a second-order differential equation and as such, its solutions will be dependent on two initial conditions for the beam. These are the size and slope of the envelope at the entrance to the ion channel, denoted by $p_{i}$ and $p_{i}$ '. The location of the channel entrance is denoted $x_{i}$. Since the motivation for this work is to understand the behavior of beams in radiographic accelerators, we find it more convenient to work in terms of the unperturbed (ion-free) beam that we would like to have. We define a nominal beam to be an emittancedominated, ion-free beam whose envelope is given by equation (3.2); thus the normalized entrance conditions are given by:

$$
\begin{aligned}
& p_{i}^{2}=p_{o}^{2}\left(1+\frac{\left(x_{i}-x_{w}\right)^{2}}{p_{o}^{4}}\right) \\
& {p_{i}^{\prime}}^{2}=\frac{1}{p_{o}^{2}} \frac{\left(x_{i}-x_{w}\right)^{2} / p_{o}^{2}}{\left(p_{o}^{2}+\left(x_{i}-x_{w}\right)^{2} / p_{o}^{2}\right)}
\end{aligned}
$$

The unperturbed waist size is $\mathrm{p}_{\mathrm{o}}$ and its location is $\mathrm{x}_{\mathrm{w}}$. In terms of a nominal beam, the entrance conditions at $x_{i}$ are uniquely determined by choosing $x_{i}$ and $p_{o}$. The time dependence of the system is captured in the time dependence of $x_{i}$ as the ion channel expands upstream into the unperturbed beam. Finally, it will be useful at some points to 
allow the location of the target plane, which serves as the ion emission surface, to be different than the location of the unperturbed beam waist. Thus we also introduce the location $\mathrm{x}_{\mathrm{t}}$ of the target plane and the notation $\mathrm{x}_{\mathrm{c}}$ for the ion channel length, where $\mathrm{x}_{\mathrm{c}}=$ $\left|\mathrm{x}_{\mathrm{t}}-\mathrm{x}_{\mathrm{i}}\right|$; we will also use the shorthand $\mathrm{x}_{\mathrm{iw}}$ for $\left|\mathrm{x}_{\mathrm{i}}-\mathrm{X}_{\mathrm{w}}\right|$.

Inspection of the detailed solutions provided in Appendix A shows that the explicit dependence of those solutions on the initial conditions is on $p_{i}$ and a combination of $p_{i}$ and $\mathrm{p}_{\mathrm{i}}$ ' given by:

$$
C \equiv p_{i}^{\prime 2}+\ln p_{i}^{2}+\frac{1}{p_{i}^{2}}
$$

A useful property of a nominal beam is:

$$
p_{i}^{\prime 2}+\frac{1}{p_{i}^{2}}=\frac{x_{i w}^{2} / p_{o}^{2}}{p_{o}^{4}+x_{i w}^{2}}+\frac{p_{o}^{2}}{p_{o}^{4}+x_{i w}^{2}}=\frac{1}{p_{o}^{2}}
$$

which means that:

$$
C=\frac{1}{p_{o}^{2}}+\ln p_{o}^{2}\left(1+x_{i w}^{2} / p_{o}^{4}\right)
$$

Thus, $\mathrm{C}$ varies only slowly with time, through the logarithm of the time-dependent $\mathrm{x}_{\mathrm{iw}}$. Note that it is not dependent on $\mathrm{x}_{\mathrm{c}}$.

We now proceed to examine particular characteristics of the beam as a function of $p_{o}$ and channel length. In most cases we will consider the limits $\mathrm{p}_{\mathrm{o}}{ }^{2}<<1$ and $\mathrm{p}_{\mathrm{o}}{ }^{2}>>1$ (and the squared dependence means that we are excluding a fairly small region about $\mathrm{p}_{\mathrm{o}} \sim 1$, say $\left.0.3<\mathrm{p}_{\mathrm{o}}<3\right)$.

\subsection{Minima and Maxima}

The recursion relations (A.1.2) for $\mathrm{p}_{\max }$ and $\mathrm{p}_{\min }$, the normalized extrema of the beam envelope, converge quickly for $\mathrm{C}$ greater than about 2 . Two iterations and simple Taylor expansions show that:

$$
\begin{aligned}
p_{\text {max }}^{2} & \approx p_{i}^{2} e^{1 / p_{o}^{2}} & & \mathrm{p}_{\mathrm{o}}{ }^{2}<<1 \text { or } \mathrm{x}_{\mathrm{iw}}>>\mathrm{p}_{\mathrm{o}}{ }^{2} \\
& \approx p_{i}^{2} e^{\left(x_{i w}^{2} / p_{o}^{6}+1 / p_{o}^{4}\right)}, & & \mathrm{p}_{\mathrm{o}}{ }^{2}>>1 \text { and } \mathrm{x}_{\mathrm{iw}}<<\mathrm{p}_{\mathrm{o}}{ }^{2} .
\end{aligned}
$$

Note that in the limit $\mathrm{p}_{\mathrm{o}}{ }^{2}<<1, \mathrm{p}_{\max }$ becomes exponentially large. In both limits it is proportional to $\mathrm{p}_{\mathrm{i}}$, which for a nominal beam will develop linear time dependence for an ion channel that expands at constant speed.

Two iterations and some Taylor expansions work for $\mathrm{p}_{\min }$ as well. The limiting behaviors are: 


$$
\begin{array}{ll}
p_{\text {min }}^{2} \approx \frac{p_{o}^{2}}{1+p_{o}^{2} \ln p_{i}^{2} / p_{o}^{2},} & \mathrm{p}_{\mathrm{o}}{ }^{2}<<1 ; \\
p_{\text {min }}^{2} \approx \frac{1}{\ln \left(p_{i}^{2} \ln \left(p_{i}^{2} \ln p_{i}^{2}\right)\right),} \quad & \mathrm{p}_{\mathrm{o}}{ }^{2}>>1 ;
\end{array}
$$

In the $\mathrm{p}_{\mathrm{o}}{ }^{2}<<1$ case, the minimum pinch size varies only slowly with time (with the $\log$ of $p_{i}$ ) and in most cases never get much smaller than the unperturbed value $p_{o}$. In the $p_{o}{ }^{2}$ $\gg 1$ case, the minimum pinch size is immediately much less than $p_{o}$, but still does not vary quickly and it is difficult to achieve $\mathrm{p}_{\min }<<1$ in this limit.

\subsection{Angular Spread}

The RMS value of the angular spread of the electrons in the beam is given by:

$$
\theta_{r m s}^{2}=\epsilon^{2} / R_{o}^{2}
$$

where in this context $R_{o}$ is a point along the envelope with zero slope. A large angular spread at the target plane of a radiographic accelerator is undesirable since it will reduce the forward dose in favor of scatter to larger angles. If the target plane remains within a small fraction of a wavelength from the location of $R_{\min }$ in the presence of ions, then the angular spread can be approximated in terms of the unperturbed conditions (denoted $\theta_{\mathrm{o}}{ }^{2}$ ) by:

$$
\theta_{r m s}^{2} \approx \theta_{o}^{2} p_{o}^{2} / p_{\min }^{2}
$$

Recall that we are invoking an assumption of constant emittance. Since $p_{\min }$ must be less than $\mathrm{p}_{\mathrm{o}}$, the effect is to always increase the angular spread. In the $\mathrm{p}_{\mathrm{o}}^{2}<<1$ limit, the increase is small and slowly varying:

$$
\theta_{r m s}^{2} \approx \theta_{o}^{2}\left(1+p_{o}^{2} \ln p_{i}^{2} / p_{o}^{2}\right), \quad \quad \mathrm{po}_{o}^{2}<<1
$$

In the $\mathrm{p}_{\mathrm{o}}^{2}>>1$ limit, the increase in angular spread is more severe:

$$
\theta_{r m s}^{2} \approx \theta_{o}^{2} \ln \left(p_{i}^{2} \ln \left(p_{i}^{2} \ln p_{i}^{2}\right)\right), \quad \quad \mathrm{po}_{\mathrm{o}}^{2}>>1
$$

In this case, stronger time variation will be introduced by the relative motion of the pinched waist relative to the target plane; this is discussed in detail in a later section. We note here that the angular spread actually decreases if the target plane is close to $p_{\max }$ :

$$
\theta_{r m s}^{2} \approx \theta_{o}^{2} e^{-1 / p_{o}^{2}} /\left(1+x_{i w}^{2} / p_{o}^{4}\right), \quad \mathrm{p}_{o}^{2}>>1, \text { target near } \mathrm{p}_{\max }
$$


The constant-emittance assumption, however, should be questioned in the presence of such strong variations in the beam envelope.

\subsection{Emergence of the Minimum}

Let us consider the behavior of the beam at the fixed location of the target plane, $\mathrm{x}=\mathrm{x}_{\mathrm{t}}$, and allow $x_{i}$ to vary with time. In this frame of reference, the various general features of the envelope that were described at the beginning of section 5 will "emerge" one by one from the target plane. We assume that the unperturbed waist of the nominal beam is coincident with the target plane $\left(\mathrm{x}_{\mathrm{t}}=\mathrm{x}_{\mathrm{W}}\right)$; the scaling for an under-focused beam $\left(\mathrm{x}_{\mathrm{W}}>\mathrm{x}_{\mathrm{t}}\right)$ is easily found relative to the $\mathrm{x}_{\mathrm{t}}=\mathrm{x}_{\mathrm{W}}$ results, as we show in Section 7, on spot control. We will not address an over-focused $\left(\mathrm{x}_{\mathrm{w}}<\mathrm{x}_{\mathrm{t}}\right)$ case. Since $\mathrm{x}_{\mathrm{t}}=\mathrm{x}_{\mathrm{w}}$, the first feature to emerge will be $\mathrm{p}_{\mathrm{min}}$, as the beam pinches down from its unperturbed size.

\subsection{1. $\mathrm{p}_{\mathrm{o}}<<1$}

To find the channel length at the time of emergence in the limit $\mathrm{p}_{\mathrm{o}}<<1$, we will look for solutions with $\mathrm{p}_{\mathrm{i}} \sim \mathrm{p}_{\mathrm{o}}$ (an assumption to be verified at the end) and solve:

$$
x_{i w}=\left|x_{m i n}-x_{i}\right|=\int_{p_{\text {min }}}^{p_{i}} \frac{d p}{\sqrt{C-1 / p^{2}-\ln p^{2}}}
$$

where the first equation defines the condition for emergence (using $\mathrm{x}_{\mathrm{W}}=\mathrm{x}_{\mathrm{t}}$ ) and the second is the normalized quadrature form that is the starting point for the solution in Appendix A. In this case, however, we are not interested in capturing the envelope for arbitrary values of $\mathrm{p}$ and can therefore use a different approach (and one that is considerably more transparent than that of the Appendix). Using the definitions of $\mathrm{C}$ and $\mathrm{p}_{\min }$, we can rewrite the integral in the form:

$$
\left|x_{m i n}-x_{i}\right|=\int_{p_{m i n}}^{p_{i}} \frac{p_{\text {min }} d p}{\sqrt{1-p_{m i n}^{2} / p^{2}-p_{m i n}^{2} \ln \left(p^{2} / p_{m i n}^{2}\right)}}
$$

For a useful range of $\mathrm{p}$, the last term in the square root can be treated as perturbation:

$$
\left|x_{m i n}-x_{i}\right| \approx \int_{p_{m i n}}^{p_{i}} p_{m i n} d p\left(\frac{1}{\sqrt{1-p_{m i n}^{2} / p^{2}}}+\frac{p_{m i n}^{2} \ln \left(p^{2} / p_{\min }^{2}\right)}{2\left(1-p_{m i n}^{2} / p^{2}\right)^{3 / 2}}\right)
$$

It is interesting to note that the second term is still singular - but Taylor-expanding about the $\mathrm{p}=\mathrm{p}_{\text {min }}$ limit shows it is the same square-root singularity as the first term, and it is therefore justifiable to call it a perturbation. The term will order like $\mathrm{p}_{\min }{ }^{2} \sim \mathrm{p}_{\mathrm{o}}{ }^{2}$ until the logarithm becomes large, which requires an exponentially long normalized channel length:

$$
p_{o}^{2} \ln \left(p_{i}^{2} / p_{o}^{2}\right) \sim 1 \rightarrow x_{i w} \sim p_{o}^{2} e^{1 / 2 p_{o}^{2}}
$$


Using the change of variable:

$$
y \equiv \sqrt{\frac{p^{2}}{p_{\min }^{2}}-1}
$$

We may quickly integrate the first term and write:

$$
\left|x_{\min }-x_{i}\right| \approx p_{\min }^{2} \sqrt{\frac{p_{i}^{2}}{p_{\min }^{2}}-1}+\frac{p_{\min }^{4}}{2} \int_{0}^{\sqrt{p_{i}^{2} / p_{\min }^{2}-1}} d y \frac{\left(y^{2}+1\right) \ln \left(y^{2}+1\right)}{y}
$$

Invoking the assumption of $\mathrm{p}_{\mathrm{i}} \sim \mathrm{p}_{\mathrm{o}}$ at emergence, we can approximate the integrand for $\mathrm{y}$ $\sim 1$ by:

$$
\frac{\left(y^{2}+1\right) \ln \left(y^{2}+1\right)}{y} \approx 1+y^{2} / 2-y^{3} / 8
$$

Integrating and using (5.2.1), we find to $\mathrm{O}\left(\mathrm{p}_{\mathrm{o}}{ }^{2}\right)$ :

$$
\begin{aligned}
& \sqrt{p_{i}^{2}-p_{\text {min }}^{2}} \approx \frac{\left|x_{i w}\right|}{p_{o}}\left(1+\frac{p_{o}^{6}}{2 x_{i w}^{2}} \ln p_{i}^{2} / p_{o}^{2}\right) \\
& 1+\frac{x_{i w}^{2}}{6 p_{o}^{4}}-\frac{x_{i w}^{3}}{32 p_{o}^{6}}=\left(1-\frac{p_{o}^{4}}{x_{i w}^{2}}\right) \ln \left(1+x_{i w}^{2} / p_{o}^{4}\right)
\end{aligned}
$$

The latter equation is only a function of $\mathrm{x}_{\mathrm{iw}} / \mathrm{p}_{\mathrm{o}}{ }^{2}$ and may be solved numerically to yield:

$$
x_{i w, \text { emerge }} \approx 2.3 p_{o}^{2}
$$

This confirms the starting assumptions of $\mathrm{x}_{\mathrm{iw}} \sim \mathrm{p}_{\mathrm{o}}{ }^{2}$ ordering and $\mathrm{p}_{\mathrm{i}}\left(\mathrm{x}_{\mathrm{iw}}\right) \sim \mathrm{p}_{\mathrm{o}}$. Thus, in the $\mathrm{p}_{\mathrm{o}}<<1$ limit, even very short ion channels can put the beam into an expansion phase. As will be seen later, however, the expansion rate is not necessarily high and there is a delay before it reaches a steady value.

Figure 5.4.1.1 shows a comparison of the above limiting expression, the "full analytic" result from Appendix A, and a completely numerical solution; the channel length at emergence is denoted $\mathrm{x}_{\mathrm{e}}$. 


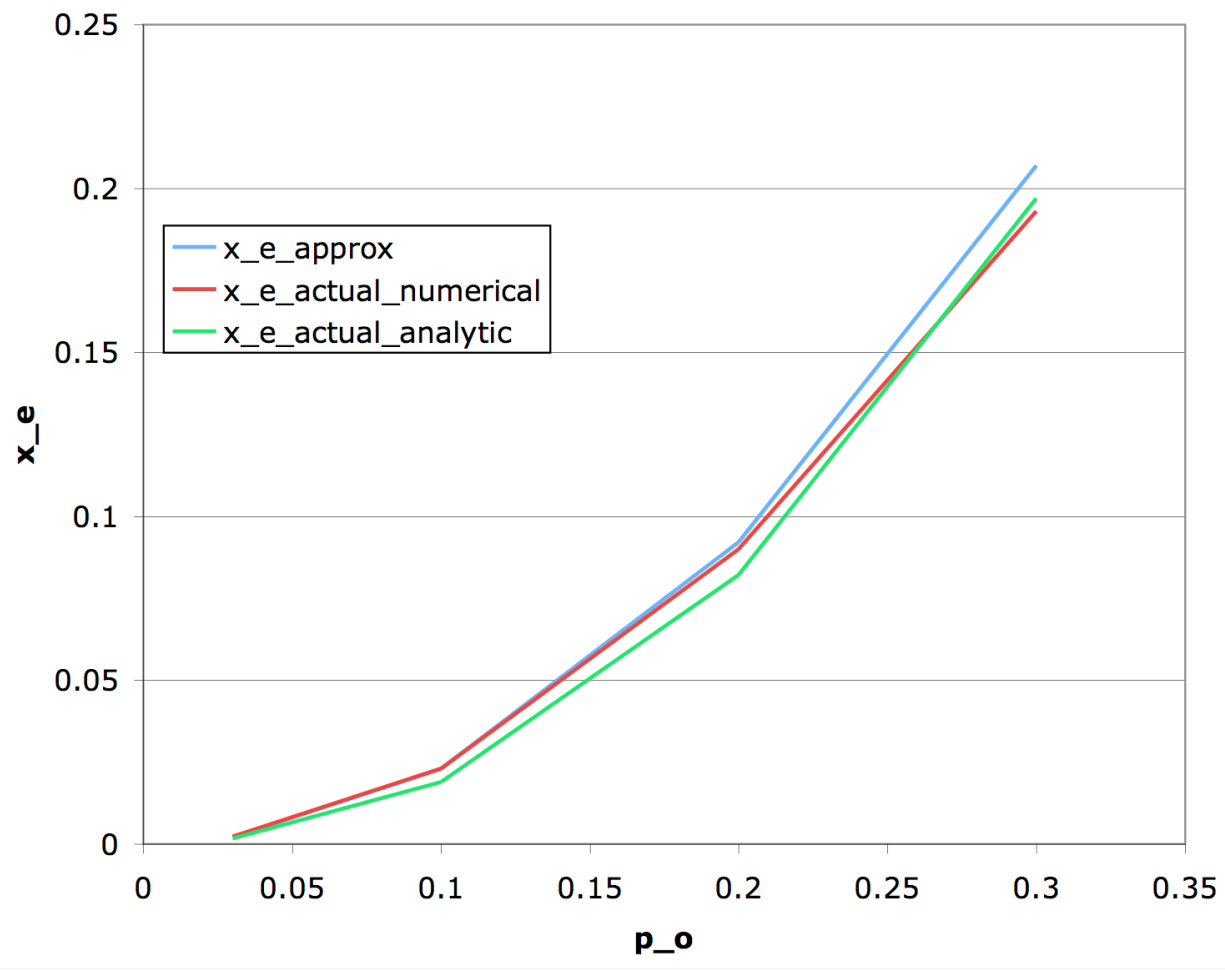

Figure 5.4.1.1. Comparison of the limiting form (blue) with numerical solutions to the full analytic model (green) and a full numerical solution to the original ODE (red), for the channel length at the emergence of the minimum spot in the $p_{0}<1$ limit.

\subsection{2. $\mathrm{p}_{\mathrm{o}}>>1$.}

In the $\mathrm{p}_{\mathrm{o}}>>1$ limit, $\mathrm{p}_{\mathrm{i}}>1$ by definition, for any channel length. Emergence is defined by:

$$
x_{i w}=\left|x_{m i n}-x_{i}\right|=\left|x_{1}-x_{i}\right|+\left|x_{\min }-x_{1}\right|
$$

We find $\mathrm{x}_{1}-\mathrm{x}_{\mathrm{i}}$ by finding the limiting form of equation (A.3.3):

$$
\left|x_{1}-x_{i}\right|=\frac{\left(I_{i}+I_{1}\right)\left(t_{i}-t_{1}\right)}{2}
$$

We expect that $\mathrm{x}_{\mathrm{iw}, \text { emerge }}$ will be $<<\mathrm{p}_{\mathrm{o}}{ }^{2}$ but still $>>1$ in this limit. The leading order behavior of a host of intermediate quantities is thus:

$$
\begin{aligned}
& p_{\text {max }}^{2} \approx p_{o}^{2}\left(1+x_{i w}^{2} / p_{o}^{4}+x_{i w}^{2} / p_{o}^{6}\right) \\
& C \approx x_{i w}^{2} / p_{o}^{4}+1 / p_{o}^{2}+\ln p_{o}^{2} \\
& s_{\max } \approx p_{o}\left(1+x_{i w}^{2} / 2 p_{o}^{4}-p_{\min }^{2} / 2 p_{o}^{2}\right)
\end{aligned}
$$




$$
\begin{aligned}
& s_{i} \approx x_{i w} / p_{o}^{2} \\
& s_{1} \approx p_{o}\left(1+x_{i w}^{2} / 2 p_{o}^{4}-1 / 2 p_{o}^{2}\right) \\
& t_{i} \approx \sqrt{p_{o}}\left(1-x_{i w} / 2 p_{o}^{3}+x_{i w}^{2} / 4 p_{o}^{4}-p_{m i n}^{2} / 4 p_{o}^{2}\right) \\
& t_{1} \approx \sqrt{\left(1-p_{\text {min }}^{2}\right) / 2 p_{o}}
\end{aligned}
$$

The highest-order term in $\mathrm{p}_{\max }{ }^{2}$ is shown only to facilitate the correct calculation of $\mathrm{s}$. To order $1 / p_{o}$,

$$
\begin{aligned}
& \left|x_{1}-x_{i}\right|=\left(\frac{t_{1} s_{1}}{\sqrt{C-1}}+\frac{t_{i} s_{i}}{\left|p_{i} p_{i}^{\prime}\right|}\right)\left(t_{i}-t_{1}\right) \\
& \approx\left(\frac{\sqrt{p_{o}} \sqrt{\left(1-p_{\text {min }}^{2}\right) / 2}}{\sqrt{\ln p_{o}^{2}-1}}+\frac{\sqrt{p_{o}}\left(x_{i w} / p_{o}^{2}\right)}{\left(x_{i w} / p_{o}^{2}\right)}\right)\left[\sqrt{p_{o}}\left(1-\frac{1}{p_{o}} \sqrt{\left(1-p_{\text {min }}^{2}\right) / 2}\right)\right] \\
& =p_{o}\left[1+\sqrt{\frac{1-p_{\text {min }}^{2}}{2}}\left(\frac{1}{\sqrt{\ln p_{o}^{2}-1}}-\frac{1}{p_{o}}\right)\right]
\end{aligned}
$$

For the remaining piece from $\mathrm{x}_{1}$ to $\mathrm{x}_{\min }$, we begin with (A.2.4) evaluated at $\mathrm{p}=1$ :

$$
\cos 2 \sqrt{\alpha}\left|x_{m i n}-x_{1}\right|=1+\frac{2 p_{\text {min }}^{2}}{p_{\text {eff }}^{2}}-\frac{2}{p_{\text {eff }}^{2}}
$$

$p_{\text {eff }}^{2}$ is only moderately larger than one in this limit, but we proceed with a Taylor expansion nonetheless:

$$
1-2 \alpha\left(x_{\min }-x_{1}\right)^{2}=1-2\left(1-p_{\min }^{2}\right) / p_{\text {eff }}^{2}
$$

Substituting for $\alpha$ and rearranging,

$$
\frac{1-p_{\min }^{2}}{p_{\min }^{2}\left(1-p_{\min }^{2} / p_{\text {eff }}^{2}\right)}\left(x_{m i n}-x_{1}\right)^{2}=1-p_{\min }^{2}
$$

Which gives the leading-order behavior of:

$$
\left|x_{\min }-x_{1}\right| \approx p_{\min }
$$

This is a small correction to (5.4.2.1) and we shall ignore it. While $\mathrm{p}_{\min }<<\mathrm{p}_{0}$, it is not necessarily $<<1$ in this limit, but to simplify (5.4.2.1) further we shall ignore it in that expression as well, leaving the leading-order behavior: 


$$
x_{i w, \text { emerge }} \approx p_{o}\left(1+\frac{1}{\sqrt{2\left(\ln p_{o}^{2}-1\right)}}-\frac{1}{\sqrt{2} p_{o}}\right), \quad p_{o}>>1
$$

Figure 5.4.2.1 shows the comparison of this expression with the full analytic and full numerical solutions.

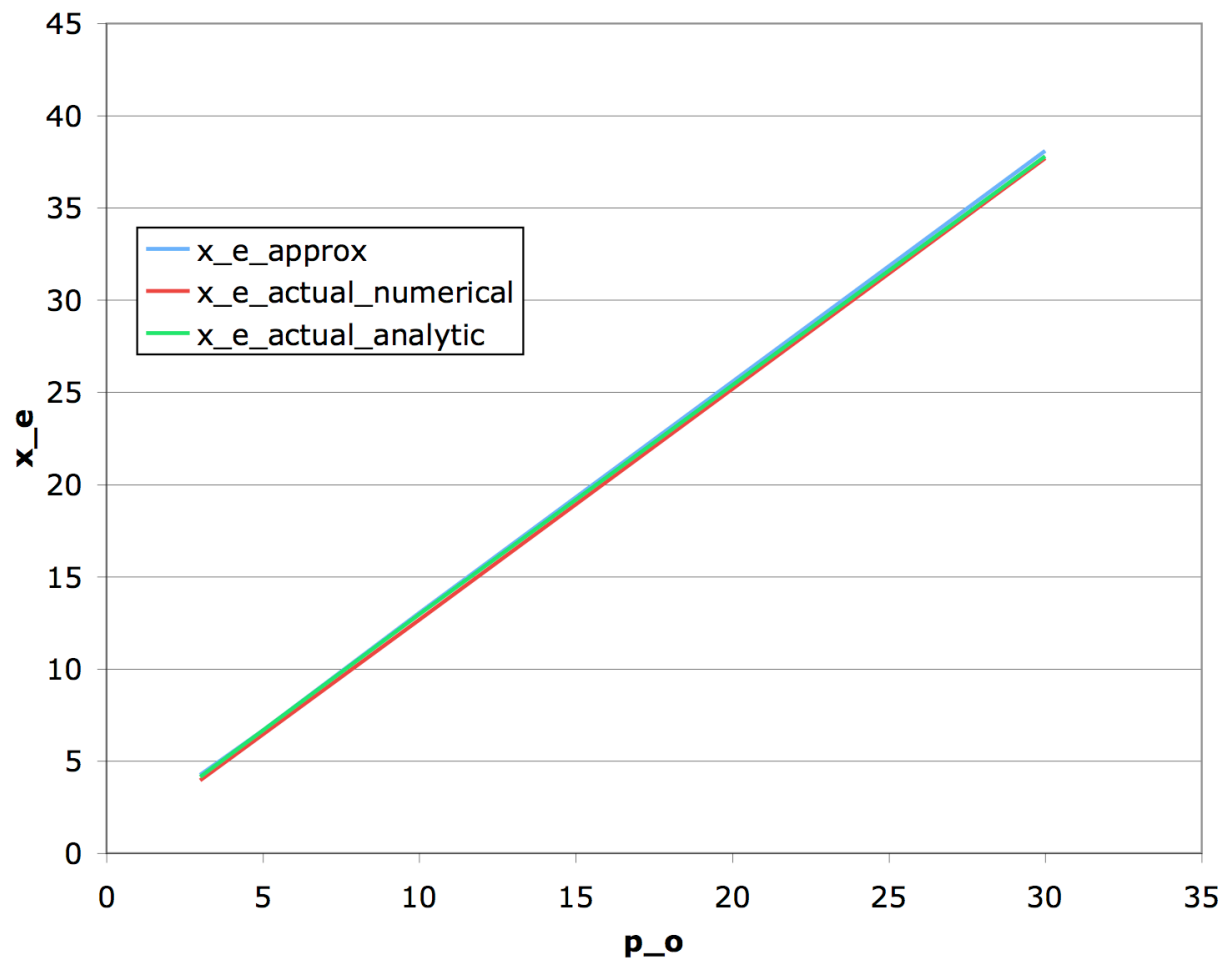

Figure 5.4.2.1. Comparison of the limiting form (blue) with numerical solutions to the full analytic model (green) and a full numerical solution to the original ODE (red), for the channel length at the emergence of the minimum spot in the $p_{0} \gg 1$ limit.

\subsection{Disruption Length}

Once the minimum emerges from the target plane, the next feature of interest that "emerges" from the target is the point on the envelope that is equal to the unperturbed spot size. Even though the beam is already in an expansion phase at this point, the same physical spot size is restored and the angular distribution is either only slightly changed (in the $p_{o}<<1$ limit) or restored since $p_{\max } \sim p_{o}$ (in the $p_{o}>>1$ limit and assuming the beam emittance survived the intervening sharp bounce). Thus, one could argue that the disruption length is a measure of the largest tolerable ion channel relative to completely ion-free behavior, and is therefore a quantity of interest; we see later that this is true more for $\mathrm{p}_{\mathrm{o}}>>1$ than $\mathrm{p}_{\mathrm{o}}<<1$. 


\subsection{1. $\mathrm{p}_{\mathrm{o}} \gg 1$}

We saw that the minimum spot size emerges from the target plane in the $p_{o}>>1$ limit when the size of the beam at the channel entrance is still not much larger than $p_{o}$. Since none of the orderings in that calculation change so long as the ion channel length is still of order $p_{0}$, and since the envelope is symmetric about $\mathrm{p}_{\min }$, we can write down an approximation for the disruption length by simply doubling the emergence length. The entrance size will still be of order $p_{o}$ and the symmetry argument means the beam size on the target will also be close to $p_{o}$. To capture an order $1 / p_{o}$ correction, we need to subtract from our estimate the distance the beam travels when expanding from $p_{o}$ to $p_{i}$ near the target:

$$
\left|x_{i w}-x_{o}\right| \approx \frac{\left(I_{i}+I_{o}\right)\left(t_{i}-t_{o}\right)}{2}
$$

Working through the intermediate quantities,

$$
\begin{aligned}
& s_{o}=\sqrt{p_{\text {max }}^{2}-p_{o}^{2}} \approx x_{i w} / p_{o}^{2} \\
& t_{o}=\sqrt{s_{\max }-s_{o}} \approx \sqrt{p_{o}}\left(1-x_{i w} / 2 p_{o}^{2}\right) \\
& I_{o} / 2=\frac{t_{o} s_{o}}{\sqrt{p_{o}^{2}\left(C-\ln p_{o}^{2}\right)-1}} \approx \frac{1}{\sqrt{p_{o}}}\left(1-x_{i w} / 2 p_{o}^{2}\right)
\end{aligned}
$$

Which gives to order $1 / \mathrm{p}_{\mathrm{o}}$ :

$$
\begin{aligned}
& x_{\text {iw, disrupt }} \approx 2 x_{\text {iw,emerge }}-\left|x_{i w}-x_{o}\right| \\
&=2 p_{o}\left(1+\frac{1}{\sqrt{2}}\left(\frac{1}{\sqrt{\ln p_{o}^{2}-1}}-\frac{1}{p_{o}}\right)\right)-\frac{x_{i w, \text { disrupt }}}{2 p_{o}} \\
&=2 p_{o}\left(1+\frac{1}{\sqrt{2\left(\ln p_{o}^{2}-1\right)}}-\frac{1+\sqrt{2}}{2 p_{o}}\right), \quad p_{o}>>1
\end{aligned}
$$

Figure 5.5.1.1 shows the usual comparisons. 


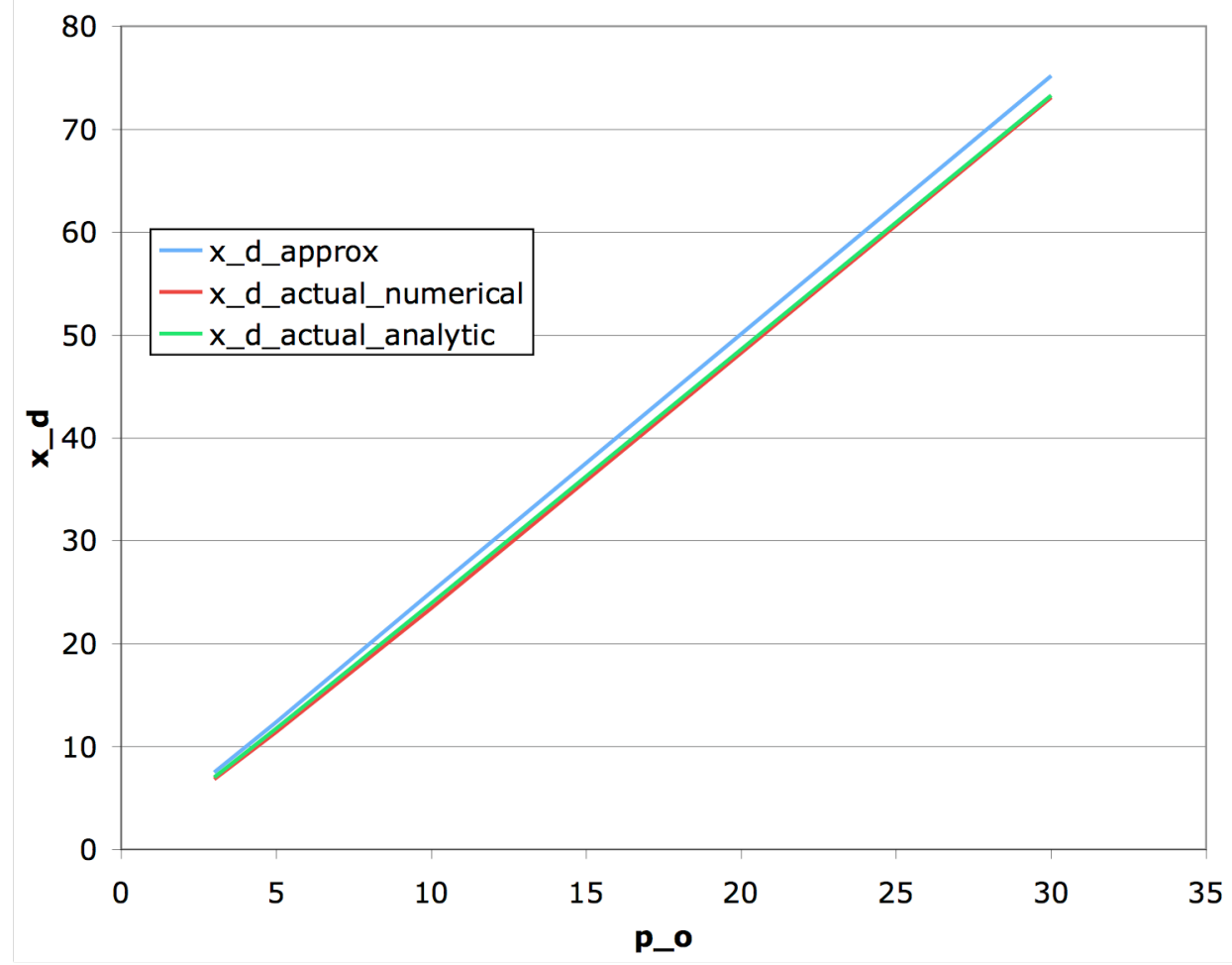

Figure 5.5.1.1. Comparison of the limiting form (blue) with numerical solutions to the full analytic model (green) and a full numerical solution to the original ODE (red), for the disruption length in the $p_{0}>>1$ limit.

\subsection{2. $\mathrm{p}_{0}<<1$}

The $\mathrm{p}_{\mathrm{o}}<<1$ case requires more work. The size of the beam at the channel entrance changes rapidly with channel length so that symmetry about the minimum is not useful. The first step is to find the position of the minimum for times after emergence. We consider equation (5.4.1.1) again, only now for the case $\mathrm{p}_{\mathrm{i}}^{2} \sim 1>>\mathrm{p}_{\mathrm{o}}^{2}$. This implies we are mostly interested in $\mathrm{y}>>1$ and we approximate the integrand in that region:

$$
\left|x-x_{\min }\right| \approx p_{\min }^{2} \sqrt{\frac{p^{2}}{p_{\min }^{2}}-1}+\frac{p_{\min }^{4}}{2} \int_{0}^{\sqrt{p^{2} / p_{\min }^{2}-1}} 2 \ln y d y
$$

Retaining terms to $\mathrm{O}\left(\mathrm{p}_{\mathrm{o}}{ }^{2}\right)$, we find:

$$
\begin{aligned}
& p^{2} \approx \frac{x_{i w}^{2}}{p_{o}^{2}}\left(1+p_{o}^{4} / x_{i w}^{2}\right) \\
& p_{\text {min }}^{2} \approx p_{o}^{2}\left(1-p_{o}^{2} \ln x_{i w}^{2} / p_{o}^{4}\right) \\
& \frac{p^{2}}{p_{\text {min }}^{2}}-1 \approx \frac{x_{i w}^{2}}{p_{o}^{4}}\left(1+p_{o}^{2} \ln x_{i w}^{2} / p_{o}^{4}\right)
\end{aligned}
$$




$$
\left|x-x_{\min }\right| \approx\left|x_{i w}\right|\left(1-p_{o}^{2}\right)=\left|x_{i w}\right|-\left|x_{t}-x_{\min }\right|
$$

The last equality follows if the target plane coincides with the unperturbed waist, $\mathrm{x}_{\mathrm{t}}=\mathrm{x}_{\mathrm{W}}$. This leads to the very useful relation:

$$
\left|x_{t}-x_{\min }\right| \approx\left|x_{i w}\right| p_{o}^{2}
$$

For $\mathrm{y} \sim 1$ or less, the last term in equation $(5.5 .2 .1)$ is $\mathrm{O}\left(\mathrm{p}_{\mathrm{o}}{ }^{2}\right)$ relative to the preceding one and the leading-order behavior for the disruption length $\left(\mathrm{p}\left(\mathrm{x}_{\mathrm{t}}\right)=\mathrm{p}_{\mathrm{o}}\right)$ is given by:

$$
p_{\min } \sqrt{p_{o}^{2}-p_{\min }^{2}}=\left|x_{t}-x_{\min }\right|=\left|x_{i w}\right| p_{o}^{2}
$$

which solves to:

$$
x_{i w, \text { disrupt }} \approx p_{o} \sqrt{2 \ln x_{i w, \text { disrupt }} / p_{o}^{2}} \approx p_{o} \sqrt{2 \ln 1 / p_{o}}
$$

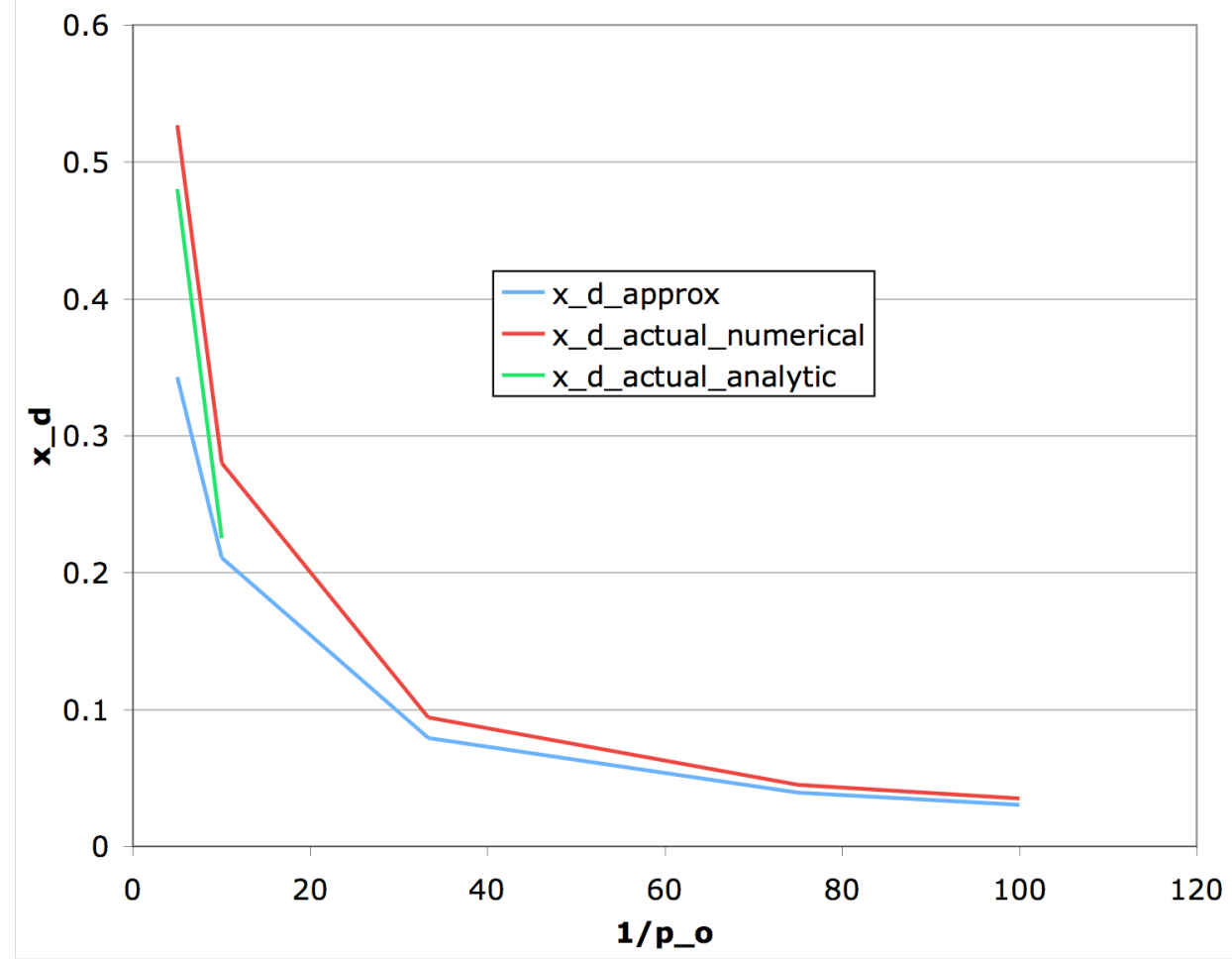

Figure 5.5.2.1. Comparison of the limiting form (blue) with numerical solutions to the full analytic model (green) and a full numerical solution to the original ODE (red), for the disruption length in the $p_{0}<1$ limit.

Figure 5.5.2.1 shows the comparisons of the last expression with the numerical and Appendix A solutions (the curve for Appendix A is truncated, since numerical evaluation 
of that model requires more care in handling very large and very small numbers than I implemented, for very small $\mathrm{p}_{\mathrm{o}}$ ).

Two points should be noted. The first is that, as hinted at by the exact examples in Section 4, the form of the disruption length is somewhat "soft" if one wants to represent it as purely linear in $p_{0}$. The second, also mentioned in section 5.4.1, is that reaching emergence or even the disruption length is still not the same as reaching the fastest growth in the $\mathrm{p}_{\mathrm{o}}<<1$ limit. We will quantify this further in the next section.

\subsection{Long-Channel Behavior}

As the ion channel grows, a nominal beam will begin to exhibit an interesting "frozen phase" phenomenon at the target plane. Even though both the wavelength and amplitude of the beam oscillation continue to evolve, the target plane ends up at a position along the wavelength that varies only slowly with time.

\section{$\underline{5.6 .1 .} \underline{p}_{\underline{o}}>>1$}

In the large $p_{o}$ limit, the ion channel is not considered "long" until the size of the beam at channel entrance has begun to grow significantly relative to the unperturbed spot, $p_{i}>p_{0}$. This requires $\mathrm{x}_{\mathrm{iw}}>\mathrm{p}_{\mathrm{o}}{ }^{2}$. Let us begin by calculating the wavelength $\lambda=2\left|\mathrm{x}_{\max }-\mathrm{x}_{\min }\right|$ in this regime. We build it out of three pieces, $\left|\mathrm{x}_{\mathrm{i}}-\mathrm{x}_{\max }\right|+\left|\mathrm{x}_{1}-\mathrm{x}_{\mathrm{i}}\right|+\left|\mathrm{x}_{\min }-\mathrm{x}_{1}\right|$; a short calculation which we will not show in detail yields that the last term is of order $p_{\text {min }}$, which, while not necessarily much less than one, is small enough to ignore in this limit.

In the long-channel regime, the correct limiting expression for $\mathrm{p}_{\max }$ (see section 5.2) is:

$$
p_{\max }^{2} \approx p_{i}^{2} e^{1 / p_{o}^{2}}
$$

The remaining contributions to the wavelength follow from some tedious but straightforward algebra, using the expressions developed in the appendix:

$$
\begin{gathered}
\left|x_{i}-x_{\max }\right|=\frac{1}{2}\left(I_{\max }+I_{i}\right)\left(t_{\max }-t_{i}\right)=\left(\frac{t_{\max }}{\sqrt{1-1 / p_{\max }^{2}}}+\frac{t_{i} s_{i} p_{o}^{2}}{x_{i w}}\right)\left(t_{\text {max }}-t_{i}\right) \\
s_{\max }=\sqrt{p_{\max }^{2}-p_{\min }^{2}} \approx p_{\max }\left(1-p_{\min }^{2} / 2 p_{\max }^{2}\right) \approx p_{i}\left(1+1 / 2 p_{o}^{2}\right) \\
t_{\max }=\sqrt{s_{\max }} \approx \sqrt{p_{i}}\left(1+1 / 4 p_{o}^{2}\right) \\
s_{i}=\sqrt{p_{\max }^{2}-p_{i}^{2}} \approx p_{i} \sqrt{e^{1 / p_{o}^{2}-1}} \approx p_{i} / p_{o} \\
t_{i}=\sqrt{s_{\max }-s_{i}} \approx \sqrt{p_{i}}\left(1-1 / 2 p_{o}\right)
\end{gathered}
$$




$$
\begin{gathered}
\left|x_{i}-x_{\max }\right| \approx\left(\sqrt{\frac{x_{i w}}{p_{o}}}+\sqrt{\frac{x_{i w}}{p_{o}}}\left(1-1 / 2 p_{o}\right)\right)\left(\sqrt{\frac{x_{i w}}{p_{o}}}-\sqrt{\frac{x_{i w}}{p_{o}}}\left(1-1 / 2 p_{o}\right)\right) \\
=\frac{x_{i w}}{p_{o}}\left(2-1 / 2 p_{o}\right)\left(1 / 2 p_{o}\right) \approx \frac{x_{i w}}{p_{o}^{2}}
\end{gathered}
$$

Note that in our converging-beam convention, $\mathrm{x}_{\max }$ denotes the position of the maximum that would occur upstream of $\mathrm{x}_{\mathrm{i}}$, if the ion channel were so extended, as in figure 5.1.

The largest contribution to the wavelength is from the remaining piece:

$$
\begin{aligned}
& \left|x_{1}-x_{i}\right|=\frac{1}{2}\left(I_{1}+I_{i}\right)\left(t_{i}-t_{1}\right)=\left(\frac{t_{1} s_{1}}{\sqrt{C-1}}+\frac{t_{i} s_{i} p_{o}^{2}}{x_{i w}}\right)\left(t_{i}-t_{1}\right) \\
& s_{1}=\sqrt{p_{\max }^{2}-1} \approx p_{i}\left(1+1 / 2 p_{o}^{2}-1 / 2 p_{i}^{2}\right) \\
& t_{1}=\sqrt{s_{\max }-s_{1}} \approx 1 / \sqrt{2 p_{i}} \\
& \left|x_{1}-x_{i}\right| \approx\left(\sqrt{\frac{p_{i}}{2}} \frac{1}{\sqrt{\ln p_{i}^{2}-1}}+\sqrt{p_{i}}\left(1-1 / 2 p_{o}\right)\right)\left(\sqrt{p_{i}}\left(1-1 / 2 p_{o}\right)-1 / \sqrt{2 p_{i}}\right) \\
& \approx p_{i}\left(\frac{1}{\sqrt{2\left(\ln p_{i}^{2}-1\right)}}+1-1 / 2 p_{o}\right)\left(1-1 / 2 p_{o}\right) \\
& \approx \frac{x_{i w}}{p_{o}}\left(1+\frac{1}{\sqrt{2\left(2 \ln \left(x_{i w} / p_{o}\right)-1\right)}}-1 / p_{o}\right)
\end{aligned}
$$

Giving the desired result:

$$
\lambda \approx 2 \frac{x_{i w}}{p_{o}}\left(1+\frac{1}{\sqrt{2\left(2 \ln \left(x_{i w} / p_{o}\right)-1\right)}}\right), \mathrm{p}_{\mathrm{o}}>1, \mathrm{x}_{\mathrm{iw}}>\mathrm{p}_{\mathrm{o}}{ }^{2}
$$

This leads to an interesting behavior: once the channel becomes long, the length of the channel as measured in wavelengths is approximately constant,

$$
x_{i w} / \lambda \approx \frac{p_{o}}{2}, \mathrm{p}_{\mathrm{o}}>>1, \mathrm{x}_{\mathrm{iw}}>\mathrm{p}_{\mathrm{o}}{ }^{2}
$$

Thus the target plane becomes a location of nearly frozen phase. To illustrate this, Figure 5.6.1.1 shows envelopes at a fixed (and large) value of $\mathrm{p}_{\mathrm{o}}$, for various channel lengths $\left(\mathrm{x}_{\mathrm{iw}}\right)$ in the long-channel regime. The $\mathrm{x}$-axis is scaled to $\mathrm{x} / \mathrm{x}_{\mathrm{iw}}$ and the $\mathrm{y}$-axis is scaled to $\mathrm{p} /\left(\mathrm{x}_{\mathrm{iw}} / \mathrm{p}_{\mathrm{o}}\right)$. The resulting curves are quite similar. An odd- and even- $\mathrm{p}_{\mathrm{o}}$ case are shown to 
illustrate the capture of different phase points implied by equation (5.6.1.2). It is an open question as to whether one could capture a point in the highly non-uniform $\mathrm{p}<1$ "quarter-cycle" or if the logarithmic variation is enough to prevent the use of such a small portion of the cycle. Points outside that region will grow in proportion to $\mathrm{p}_{\max } \sim \mathrm{p}_{\mathrm{i}} \sim$ $\mathrm{x}_{\mathrm{iw}} / \mathrm{p}_{\mathrm{o}}$; the latter could be considered a measure of the "disruption rate," which is otherwise ill-defined in the $p_{o}>>1$ limit.

Table 5.6.1.1 shows a comparison of equation (5.6.1.1) and the numerically-determined wavelength for various cases.

\begin{tabular}{|c|c|c|c|}
\hline $\mathbf{p}_{\mathbf{0}}$ & $\mathbf{x}_{\mathrm{iw}} / \mathbf{p}_{\mathbf{0}}{ }^{2}$ & $\begin{array}{l}\text { numerical } \\
\text { lambda }\end{array}$ & $\begin{array}{l}\text { approx. } \\
\text { lambda }\end{array}$ \\
\hline 5 & 10 & 127 & 129 \\
\hline 5 & 15 & 188 & 198 \\
\hline 5 & 20 & 249 & 256 \\
\hline 10 & 10 & 249 & 253 \\
\hline 10 & 15 & 371 & 376 \\
\hline 10 & 20 & 491 & 506 \\
\hline 15 & 10 & 371 & 378 \\
\hline 15 & 15 & 551 & 567 \\
\hline 15 & 20 & 732 & 756 \\
\hline
\end{tabular}

Table 5.6.1.1. Comparison of numerical and analytical results for the long-channel, large $p_{0}$ wavelength. 

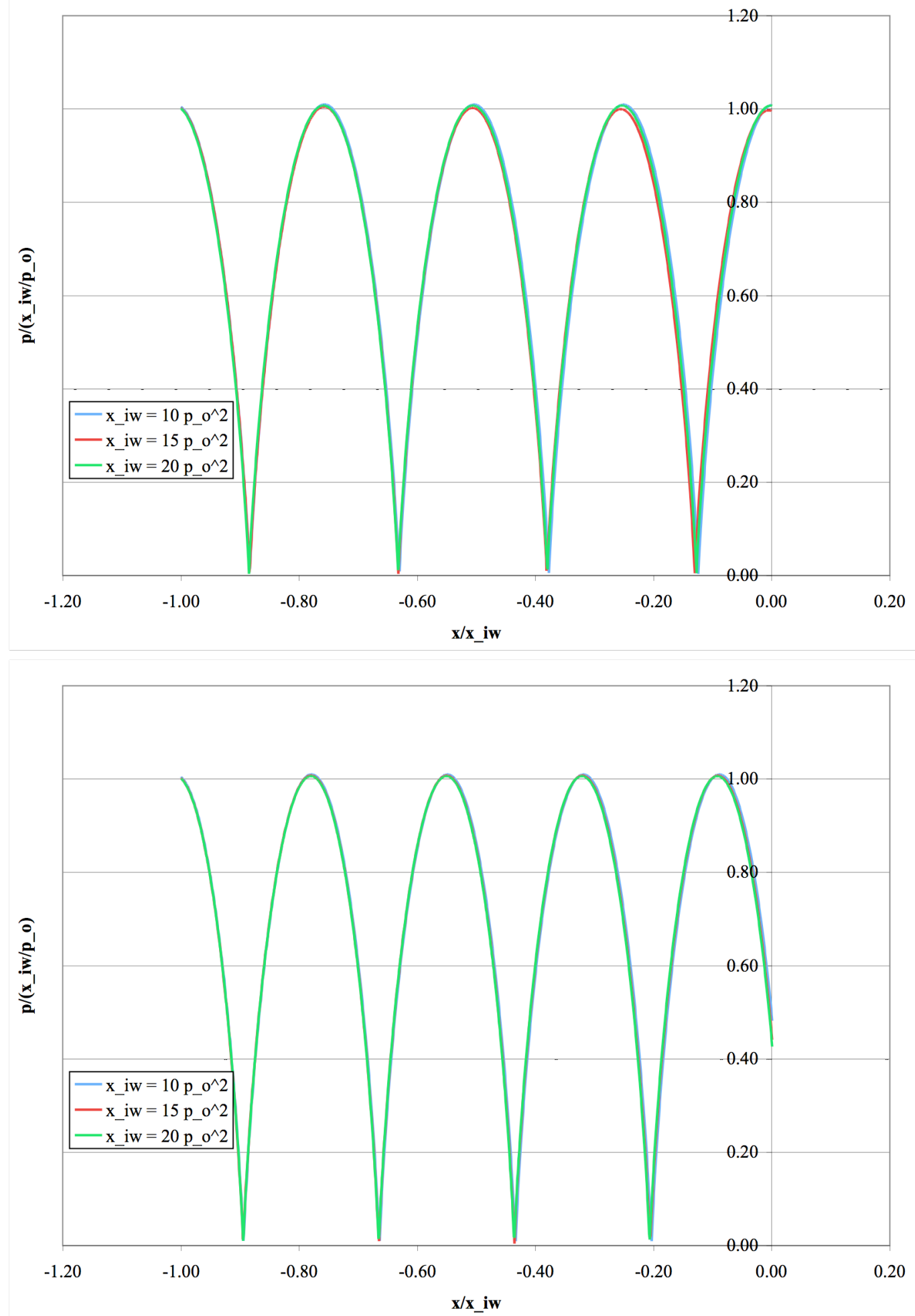

Figure 5.6.1.1. Scaled curves at fixed initial spot size $p_{0}$ and varying channel length $x_{i w}$, showing the "frozen phase" phenomenon at the target plane in the long channel, large $p_{0}$ limit. (Top) $p_{0}=10$. (Bottom) $p_{0}=11$. 


\subsection{2. $\mathrm{p}_{0}<<1$}

The long-channel behavior for $\mathrm{p}_{\mathrm{o}}<<1$ is equally interesting. Let us do the corresponding wavelength calculation, $\lambda=2\left|\mathrm{x}_{\max }-\mathrm{x}_{\min }\right|$. We begin as was done in section 5.4.1, except now we reference $p$ to $p_{\max }$ instead of $p_{\min }$ when we Taylor-expand the integrand:

$$
\left|x_{\max }-x\right|=\int_{p}^{p_{\max }} d p\left(\frac{1}{\sqrt{\ln p_{\max }^{2} / p^{2}}}-\frac{1}{2 p_{\max }^{2}} \frac{1-p_{\max }^{2} / p^{2}}{\left(\ln p_{\max }^{2} / p^{2}\right)^{3 / 2}}\right)
$$

As shown at the start of section 5.2, $\mathrm{p}_{\max }$ is exponentially large when $\mathrm{p}_{\mathrm{o}}<<1$; we will ignore the second term altogether in what follows. The calculation then becomes very similar to the zero-emittance beam case in section 4; we won't repeat all of the details of the substitutions here:

$$
\begin{aligned}
\left|x_{\max }-x\right| \approx \int_{p}^{p_{\max }} \frac{d p}{\sqrt{\ln p_{\max }^{2} / p^{2}}}=\sqrt{\frac{\pi}{2}} p_{\max } \frac{2}{\sqrt{\pi}} \int_{0}^{\sqrt{\ln p_{\max } / p}} e^{-x^{2}} d x \\
=\sqrt{\frac{\pi}{2}} p_{\max }\left(1-\operatorname{erfc}\left(\sqrt{\ln p_{\max } / p}\right)\right)
\end{aligned}
$$

For any $\mathrm{p}$ near the minimum, the value of the complementary error function will be exponentially small and we find:

$$
\lambda \approx \sqrt{2 \pi} p_{\max } \approx \sqrt{2 \pi} \frac{\left|x_{i w}\right|}{p_{o}} e^{1 / 2 p_{o}^{2}}
$$

Thus we see that the channel length $\left|\mathrm{x}_{\mathrm{iw}}\right|$ and the distance of the waist from the target plane $\left|\mathrm{x}_{\mathrm{t}}-\mathrm{x}_{\mathrm{min}}\right|$ are again "frozen" at particular fractions of a wavelength for long channels:

$$
\frac{\left|x_{t}-x_{\min }\right|}{\lambda}=\frac{p_{o}^{3}}{\sqrt{2 \pi}} e^{-1 / 2 p_{o}^{2}}, \quad \frac{\left|x_{i w}\right|}{\lambda}=\frac{p_{o}}{\sqrt{2 \pi}} e^{-1 / 2 p_{o}^{2}}
$$

The latter equation also implies that the channel length is always just a small fraction of a wavelength; i.e., in the $\mathrm{p}_{\mathrm{o}}<<1$ limit, the beam never "bounces" in terms of having a maximum emerge from the target plane.

Figure 5.6.2.1 shows numerical calculations of the beam envelope versus position, with the former normalized to $\mathrm{p}_{\mathrm{i}} \sim\left|\mathrm{x}_{\mathrm{iw}}\right| / \mathrm{p}_{\mathrm{o}}$ and the latter normalized to the channel length. Note that the curves are self-similar for a given value of $\mathrm{p}_{\mathrm{o}}$, except near $\mathrm{p}_{\min }$; and that the location of the minimum occurs at a normalized position of $\mathrm{p}_{\mathrm{o}}{ }^{2}$ (as given by (5.5.2.2)). 


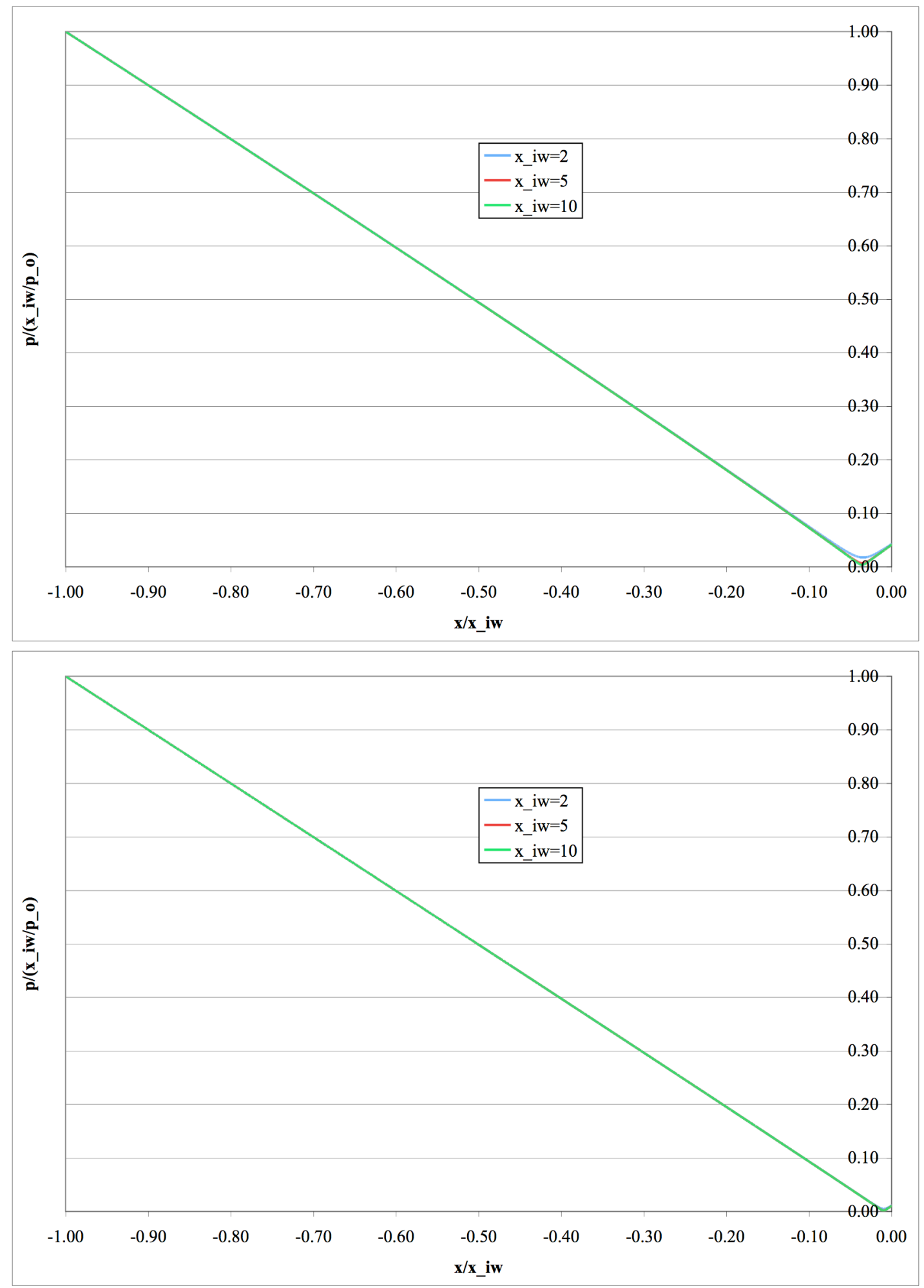

Figure 5.6.2.1. Beam envelopes (normalized to the size at the channel entrance) for various long channel lengths, showing the "freezing" of the envelope relative to the wavelength in the $p_{0}<1$ limit. Shown for $p_{0}=0.2$ (top) and 0.1 (bottom). 
Finally, we note that there are two regimes of "long" channel for the $p_{0}<<1$ case. In the first, $\left|\mathrm{x}_{\mathrm{iw}}\right| \gg>\mathrm{p}_{\mathrm{o}}$ and the formulas for the location of the minimum, the wavelength, and the disruption length apply. From the argument made for the latter in developing equation (5.5.2.3), we can write down an expression for the spot size on the target plane that is valid until $\mathrm{x}_{\mathrm{iw}} \sim 1 / \mathrm{p}_{\mathrm{o}}^{2}$ :

$$
p_{t} \approx p_{o} \sqrt{1+x_{i w}^{2}}
$$

This expression reveals that there is a second "long channel" regime when $\mathrm{x}_{\mathrm{iw}} \sim 1$. Regardless of the disruption length, the spot on target does not actually enter a linear growth phase until $\mathrm{x}_{\mathrm{iw}} \sim 1$. Figure 5.6.2.2 shows a comparison of the numerically determined spot size, normalized to $\mathrm{x}_{\mathrm{iw}} \mathrm{p}_{\mathrm{o}}$, as compared to equation (5.6.2.1), for various $\mathrm{p}_{\mathrm{o}}$.

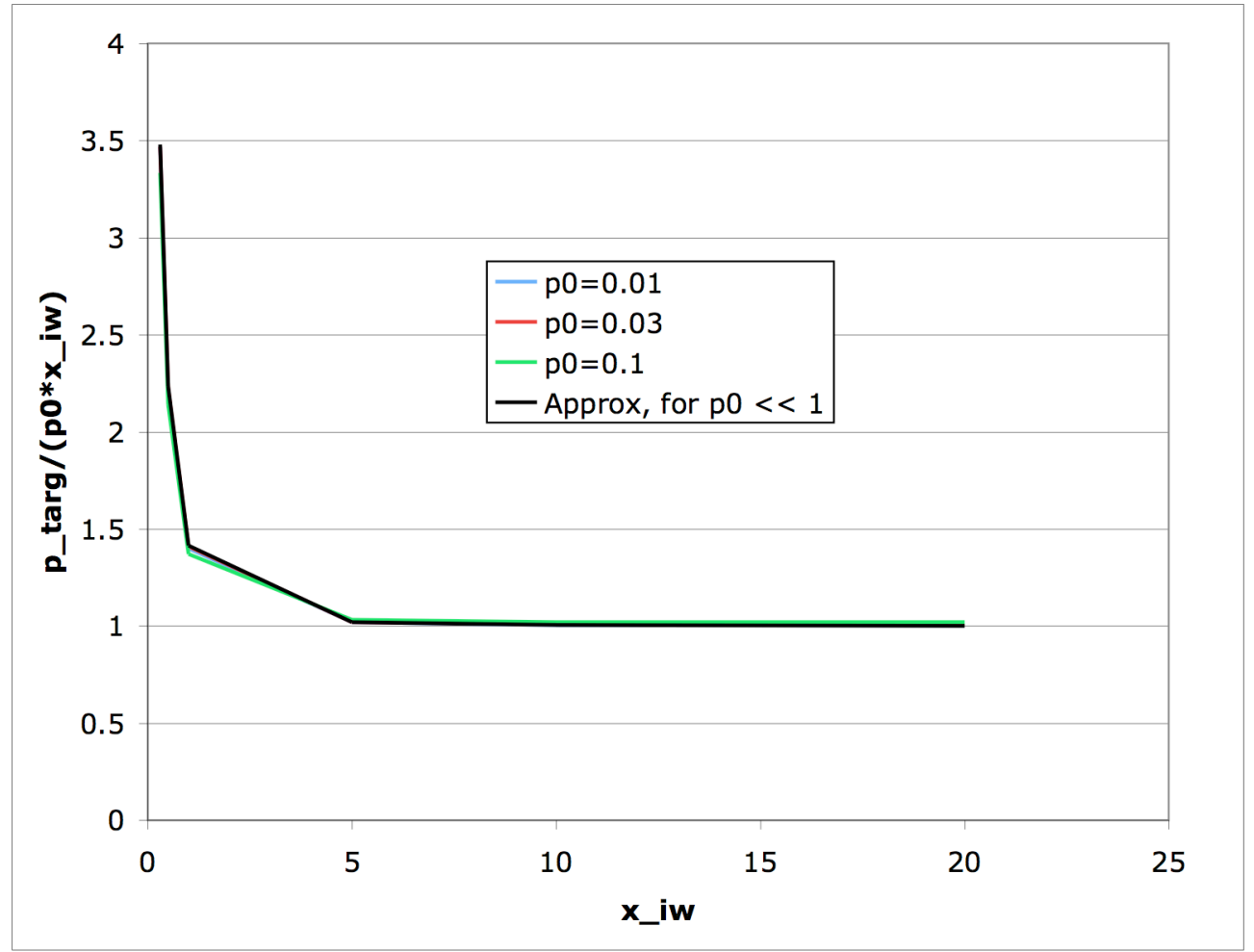

Figure 5.6.2.2. Spot size on the target plane, normalized to $\mathrm{p}_{\mathrm{o}}{ }^{*} \mathrm{x}_{\mathrm{iw}}$, compared to numerical solutions for various $\mathrm{p}_{\mathrm{o}}$ in the $\mathrm{p}_{\mathrm{o}}<<1$ limit. Note that linear growth is not actually achieved until $\mathrm{x}_{\mathrm{iw}} \sim 1$. 


\section{Validity in the Presence of a Final Focus Magnet}

Once we start to discuss the notion of a long ion channel, it begs the question about the influence of magnetic focusing. The incoming slope of the electron beam as it approaches the target has undoubtedly been produced by passage through a magnetic solenoid lens, whereas the model has heretofore assumed continued expansion of the nominal envelope out to arbitrary distances. Does the presence of a lens strongly influence the answers?

We address this question in the thin-lens limit, where the magnet is treated as supplying an impulse to the beam slope at a single position in space. We state without proof (see [10], for example) that the change in slope is linearly proportional to the beam radius entering the lens:

$$
\Delta R^{\prime}=-\Delta R_{o}^{\prime} \frac{R}{R_{e}}
$$

$\mathrm{R}_{\mathrm{e}}$ denotes the nominal beam radius entering the lens and $\Delta \mathrm{R}^{\prime}{ }_{\mathrm{o}}$ is the magnitude of the desired slope change, for an unperturbed beam. Transport upstream of the lens is generally in the space-charge dominated regime, where the normalized radius satisfies $p$ $>>1$. In the systems under consideration, over the channel lengths under consideration, the electron beam can really be considered ballistic rather than space-charge dominated. The ion channel is a larger perturbation (by a factor of $\gamma^{2}$ ) to ballistic motion that the beam space charge would be, but it is a small perturbation nonetheless.

Let us move that description to a more quantitative one. Consider an unperturbed beam focused to a waist size $R_{o}$ by a thin-lens solenoidal magnet placed at a focal length $L_{f}$ from the waist. We will limit our consideration to the case where the unperturbed beam slope is nearly zero in the region upstream of the magnet. Given ballistic behavior, the unperturbed upstream envelope magnet is simply:

$$
R(z)=R_{e}, R^{\prime}(z)=0, z>L_{f}
$$

In practice, it is almost always the case that the focal length is such that $\varepsilon L_{f} / R_{o}>>R_{o}$; or, in normalized form, $x_{f} / p_{o}>>p_{o}$, where $x_{f}=\sqrt{K L_{f}} / R_{e q}$ and $p_{o}$ is the usual unperturbed waist size in normalized form. For a nominal beam it follows that just downstream of the lens:

$$
p\left(x_{f}\right) \approx \frac{x_{f}}{p_{o}}\left(1+\frac{p_{o}^{4}}{2 x_{f}^{2}}\right), p^{\prime}\left(x_{f}^{-}\right) \approx \frac{1}{p_{o}}\left(1-\frac{p_{o}^{4}}{2 x_{f}^{2}}\right)
$$

(In this section we treat positive $\mathrm{x}$ (and $\mathrm{z}$ ) as moving upstream, away from the target and towards the lens; hence, the slope is positive and $\mathrm{x}_{\mathrm{f}}^{-}$denotes a position just downstream of $\mathrm{x}_{\mathrm{f} .}$ ) 
As a consequence of this usual mode of operation, the disruptive behavior of beams with $\mathrm{p}_{\mathrm{o}} \gg>1$ is not influenced by the presence of the lens until the beam has already been strongly perturbed by the ions. As the ion channel reaches the magnet, $\mathrm{x}_{\mathrm{iw}} \sim \mathrm{x}_{\mathrm{f}}$, it follows that $\mathrm{x}_{\mathrm{iw}} / \mathrm{p}_{\mathrm{o}}{ }^{2}>1$, which is the valid limit for equation (5.6.1.2): $\lambda / \mathrm{x}_{\mathrm{iw}} \sim \mathrm{p}_{\mathrm{o}} / 2$. Thus the beam will have already gone through many bounces in the channel before the ions "know about" the presence of the lens.

For beams with $\mathrm{p}_{\mathrm{o}}<<1$, we must be a bit more quantitative. For the perturbed beam upstream of the lens, we take a much simpler approach than used in the other sections. Far from the waist, the perturbation to beam is weak because even modest values of $\mathrm{x}_{\mathrm{f}}$ or $\mathrm{x}_{\mathrm{iw}}$ result in $\mathrm{p} \gg \mathrm{1}$. Therefore, let us work in terms of a Taylor expansion downstream of the channel, for channels extended beyond the position of the lens $\left(\mathrm{x}_{\mathrm{iw}}>\mathrm{x}_{\mathrm{f}}\right)$. This approach is valid so long as $\mathrm{x}_{\mathrm{iw}} \mathrm{p}_{\mathrm{o}}{ }^{2}<<\mathrm{x}_{\mathrm{f}}$; that is, the location of the perturbed waist (see eq. 5.5.2.2) is not close to the magnet.

We start with the case of no magnet; i.e., the beam is allowed to undergo an unbounded divergence upstream from the target. If $\mathrm{p}>>1$ then $\mathrm{x}_{\mathrm{iw}} / \mathrm{p}_{\mathrm{o}}>>1$ and we will ignore any finite-emittance corrections. Letting the subscript "i" refer to initial values at the channel entrance, we find:

$$
p_{i} \approx \frac{x_{i w}}{p_{o}}, p_{i}^{\prime} \approx \frac{1}{p_{o}}, p_{i}^{\prime \prime} \approx-\frac{1}{p_{i}} \approx-\frac{p_{o}}{x_{i w}}
$$

Using these initial values in a Taylor series gives the following leading-order behavior for the beam conditions at the location where the magnet would be:

$$
\begin{aligned}
& p\left(x_{f}\right) \approx \frac{x_{f}}{p_{o}}\left(1-\frac{p_{o}^{2}}{2} \frac{\left(x_{f}-x_{i w}\right)^{2}}{x_{i w} x_{f}}\right) \\
& p^{\prime}\left(x_{f}\right) \approx \frac{1}{p_{o}}\left(1-p_{o}^{2} \frac{x_{f}-x_{i w}}{x_{i w}}\right)
\end{aligned}
$$

Note that $\mathrm{x}_{\mathrm{f}}<\mathrm{x}_{\mathrm{iw}}$ when considering signs. Now let us put the magnet back into the problem. Under the ballistic assumption,

$$
p_{i} \approx \frac{x_{f}}{p_{o}}, p_{i}^{\prime} \approx 0, p_{i}^{\prime \prime} \approx-\frac{p_{o}}{x_{f}}
$$

At a location just upstream of the magnet, we find:

$$
p\left(x_{f}^{+}\right) \approx \frac{x_{f}}{p_{o}}\left(1-\frac{p_{o}^{2}}{2} \frac{\left(x_{f}-x_{i w}\right)^{2}}{x_{f}^{2}}\right)
$$




$$
p^{\prime}\left(x_{f}^{+}\right) \approx-p_{o} \frac{x_{f}-x_{i w}}{x_{f}}
$$

Just downstream of the magnet, $\mathrm{p}\left(\mathrm{x}_{\mathrm{f}}\right)$ does not change but the slope changes by an amount:

$$
\Delta p^{\prime}=\frac{1}{p_{o}} \frac{p\left(x_{f}\right) p_{o}}{x_{f}}
$$

Which gives:

$$
\begin{aligned}
& p\left(x_{f}^{-}\right) \approx \frac{x_{f}}{p_{o}}\left(1-\frac{p_{o}^{2}}{2} \frac{\left(x_{f}-x_{i w}\right)^{2}}{x_{f}^{2}}\right) \\
& p^{\prime}\left(x_{f}^{-}\right) \approx \frac{1}{p_{o}}\left(1-p_{o}^{2} \frac{x_{f}-x_{i w}}{x_{f}}\left(1+\frac{x_{f}-x_{i w}}{2 x_{f}}\right)\right)
\end{aligned}
$$

Thus, the behavior of the beam downstream from the magnet will be the same, to order $\mathrm{p}_{\mathrm{o}}{ }^{2}$, as a beam which continued to expand upstream from the target, with no magnet; this remains true until the perturbed waist has moved close to the magnet (at which point the Taylor-expanded behavior of the upstream portion of the beam is no longer valid). 


\section{Implications for Spot Control}

In this section, we examine some options for minimizing the effects of the ion channel on the beam focus that are based on adapting the behavior of the incoming beam; it follows from the topics considered in [6]. We note in passing that there are also many candidate options for changing the behavior of the ions, depending on the details of the applications (cleaning, blocking, biasing of the target).

\subsection{Beam-Specific Knobs (Static)}

For most of the applications where ion defocusing is a problem, we would not have the freedom of specifically choosing the beam spot size, current, energy, emittance, or pulse length for the specific purpose of avoiding significant ion-induced time variation. Nevertheless, we will discuss these knobs briefly for completeness.

To begin, we make a point of re-examining the earlier results in physical rather than normalized units. To keep the beam focal size at or below its initial value (note that we do allow the focal size to decrease; keep in mind this is at the expense of an increase in the beam angular spread), we need the channel length to remain shorter than either a disruption length $\sim 2 \mathrm{p}_{\mathrm{o}}$ for $\mathrm{p}_{\mathrm{o}}>>1$, or shorter than $\sim 1$ for $\mathrm{p}_{\mathrm{o}}<<1$.

In terms of beam parameters (as opposed to ion emission parameters such as species and charge state), the maximum channel length is a function of beam current and pulse length. The current enters by way of the ion speed, which is in turn dependent on the square-root of the potential drop in front of the target, which is in turn dependent on the current (see Section 2). For a single ion species of charge $q_{i}$ and mass $m_{i}$ :

$$
z_{i w}=\sqrt{\frac{60 q_{i}\left(1+2 \ln b / R_{o}\right)}{m_{i}}} t_{p} \sqrt{I}
$$

where $t_{p}$ is the pulse length and $b$ is the beam pipe radius (units are SI). Choosing $\mathrm{b} / \mathrm{R}_{\mathrm{o}}=10$ as a nominal value and expressing $\mathrm{q}_{\mathrm{i}}=\mathrm{Z}_{\mathrm{i}} \mathrm{q}_{\mathrm{e}}$ where $\mathrm{q}_{\mathrm{e}}$ is the fundamental electronic charge and $m_{i}=A_{i} m_{p}$ where $m_{p}$ is the mass of a proton, we can write the $x_{i w}<$ $2 \mathrm{p}_{\mathrm{o}}\left(\mathrm{p}_{\mathrm{o}}>>1\right)$ condition with all beam-specific parameters on one side, in convenient units:

$$
\frac{R_{o}(m m) \sqrt{\gamma}}{t_{p}(n s) I(k A)}>0.7 \sqrt{\frac{Z_{i} f}{A_{i}}} \quad\left(\frac{R_{o}(m m)}{\epsilon(m m m r a d)} \sqrt{\frac{I(k A)}{\gamma}}>>\frac{4 \times 10^{-3}}{\sqrt{f}}\right)
$$

It is interesting to note that so long as the right-hand $\left(\mathrm{p}_{\mathrm{o}}>>1\right)$ condition is met, the disruption length condition is not emittance-dependent.

The alternate condition $\mathrm{x}_{\mathrm{iw}}<1\left(\mathrm{p}_{\mathrm{o}}<<1\right)$ becomes: 


$$
\frac{I(k A)^{3 / 2} t_{p}(n s)}{\epsilon(m m m r a d) \gamma}<\frac{3 \times 10^{-3}}{f} \sqrt{\frac{A_{i}}{Z_{i}}} \quad\left(\frac{R_{o}(m m)}{\epsilon(m m m r a d)} \sqrt{\frac{I(k A)}{\gamma}}<<\frac{4 \times 10^{-3}}{\sqrt{f}}\right)
$$

The feature of interest in this condition is that so long as the right-hand $\left(\mathrm{p}_{\mathrm{o}}<<1\right)$ condition is met, the channel length condition is not dependent on spot size.

In practice, neither condition is easily met in existing high-intensity radiographic facilities and some other amelioration technique must be employed.

\subsection{Time-Varying Focusing}

As mentioned in section 6, "real" beams have their slope approaching the target plane set by a focusing magnet. In this section we consider the possibility of varying the focusing strength of the magnet with time in order to offset the effect of the ions. If possible, this is a desirable technique in that it represents a single modification to a transport system but we note that the ion channel perturbs both the minimum beam size and the location of that minimum; thus, to control both, one would really need two independent knobs (e.g. controlling the beam size at the magnet as well, via upstream knobs).

The approach we choose here is to adjust the slope of the beam exiting the magnet such that the perturbed waist remains on the target. In the $\mathrm{p}_{\mathrm{o}}<<1$ limit, where the minimum beam size varies only slowly as the ion channel grows, we actively preserve the location of the minimum and its size comes along "for free". In the $p_{o}>>1$ limit, the perturbed minimum beam size is quite different from the unperturbed size $\left(\mathrm{p}_{\min }\right.$ is inherently $\left.<1\right)$ and the angular spread at the minimum can be significantly higher than was originally intended; however, in the absence of a second knob, the only alternative is to try to preserve $p_{\text {targ }}=p_{o}$ without the beam being at a waist. In that case, it turns out that the phase of the beam at the target far enough from $p=p_{\max }$ that the angular spread of the beam is still increased significantly. Hence, we find it more desirable to achieve the smaller beam size on target.

\subsection{1. $\mathrm{p}_{\mathrm{o}}<<1$}

Let us begin by considering the behavior of the beam as we vary the slope at the magnet location without any perturbation from ions. In this section we want to keep the slope $\mathrm{R}^{\prime}$ a positive quantity and so we define the upstream direction to be positive $z$. We fix the target plane at $\mathrm{z}=0$ and assume a thin lens is located at $\mathrm{z}=\mathrm{L}_{\mathrm{f}}$. Denote the beam radius at the location of the magnet $R_{\text {mag }}$. From Section 3, in the limit where $R_{\min } / R_{\operatorname{mag}}<<1$, we find:

$$
\begin{aligned}
& R_{\text {min }} \approx \frac{\epsilon}{R^{\prime}} \\
& z_{\text {min }} \approx L_{f}-\frac{R_{m a g}}{R^{\prime}}
\end{aligned}
$$


We emphasize that these are the minimum beam size and location of the minimum without any ions present.

Many of the results in Section 5 were given for the case where the unperturbed beam came to a waist at the target plane, $\mathrm{z}=0$. However, all the of results can be generalized very easily by working in terms of an effective ion channel length that is referenced to the actual location of the unperturbed waist. It does not matter if the unperturbed waist is located beyond the target plane so that a portion of the ion channel is a "phantom". Thus, if we write the physical channel length in the form $v_{i} t$, where $v_{i}$ is the ion speed, the effective channel length $\mathrm{z}_{\mathrm{iw}}$ (corresponding to the normalized $\mathrm{x}_{\mathrm{iw}}$ ) is $\mathrm{v}_{\mathrm{i}} \mathrm{t}-\mathrm{z}_{\mathrm{min} \text {,un }}$, where $\mathrm{Z}_{\mathrm{min}, \text { un }}$ denotes the location of the unperturbed waist.

From equation (5.5.2.2), we find the location of the perturbed minimum relative to the unperturbed minimum:

$$
z_{m i n, p e r t}-z_{m i n, u n} \approx z_{i w} \frac{R_{m i n}^{2} K}{\epsilon^{2}}
$$

Combining this with (7.2.1.1):

$$
z_{\text {min,pert }}=\left(v_{i} t-L_{f}+\frac{R_{m a g}}{R^{\prime}}\right) \frac{K}{R^{\prime 2}}+L_{f}-\frac{R_{m a g}}{R^{\prime}}=0
$$

The latter equality is the desired condition; we must adjust $\mathrm{R}$ ' exiting the magnet to satisfy it. If we note that $\mathrm{R}_{\mathrm{mag}} / \mathrm{L}_{\mathrm{f}} \approx \mathrm{R}^{\prime}$, the slope of the unperturbed beam after the magnet, and that $\mathrm{R}^{\prime} / \sqrt{ } \mathrm{K} \equiv \mathrm{p}^{\prime}$, we get the normalized form:

$$
\left(v_{i} t / L_{f}-1\right)+p_{o}^{\prime} / p^{\prime}+p^{2}-p_{o}^{\prime} p^{\prime}=0
$$

Linearizing $\mathrm{p}^{\prime}$ to $\mathrm{p}_{\mathrm{o}}+\Delta \mathrm{p}$ ' and noting that $\mathrm{p}_{\mathrm{o}} \approx 1 / \mathrm{p}_{\mathrm{o}}$ in the $\mathrm{p}_{\mathrm{o}}<<1$ limit, we find:

$$
\Delta p^{\prime} \approx-\frac{p_{o} v_{i} t}{L_{f}}
$$

In a useful hybrid of normalized and practical units:

$$
\Delta R^{\prime} \approx-\frac{p_{o} v_{i} t \sqrt{K}}{L_{f}}
$$

Finally, writing:

$$
\Delta R^{\prime}=R^{\prime}-R_{o}^{\prime}=R_{o}^{\prime}\left(B^{2} / B_{o}^{2}-1\right) \approx R_{o}^{\prime} \frac{2 \Delta B}{B_{o}}
$$

and using $\mathrm{R}^{\prime}{ }_{\mathrm{o}} \approx \varepsilon / \mathrm{R}_{\mathrm{o}}$, we can find the condition for the time variation of the magnet field: 


$$
\Delta B=-\frac{B_{o}}{2} \frac{v_{i} t}{L_{f}} p_{o}^{2}
$$

For $300 \mathrm{kV}$ hydrogen ions, a $20 \mathrm{~cm}$ focal length, and an $0.1 \mathrm{~T}$ focusing field, this would give a time variation of $2 \times 10^{-5} \mathrm{~T} / \mathrm{ns}$ (or 0.2 gauss $/ \mathrm{ns}$ ) for $\mathrm{p}_{\mathrm{o}}=0.1$.

Figure 7.2.1.1 shows a comparison of a beam controlled by equation (7.2.1.2) with an uncontrolled beam for $\mathrm{p}_{\mathrm{o}}=0.05$.
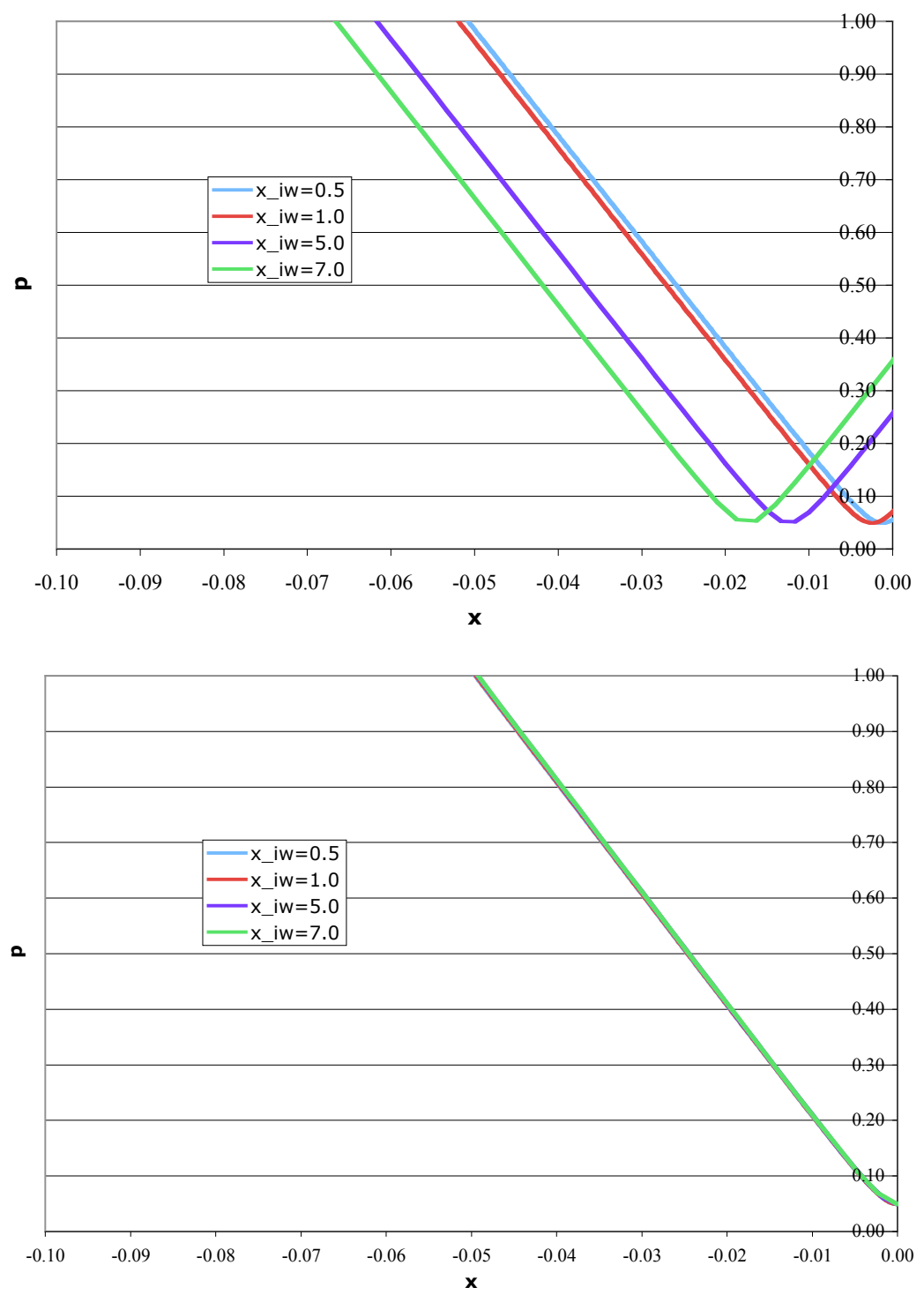

Figure 7.2.1.1. Comparison of uncontrolled spot growth as ion channel length increases (top) versus beam controlled via equation (7.2.1.2) (bottom), for $p_{0}=0.05$. 


\subsection{2. $\mathrm{p}_{0}>1$}

There is a fundamental limitation to using a time-varying magnet to control the $\mathrm{p}_{\mathrm{o}}>>1$ case. If the beam enters the magnet with nearly zero slope, then obviously the biggest change one can make is to swing from the nominal slope, at the nominal magnet setting, all the way down to zero field, meaning nearly zero slope exiting the magnet. As discussed in section 6 , the usual mode of operation is for the beam to be nearly ballistic at the magnet, which means the distance of the magnet from the target plane $\left(\mathrm{x}_{\mathrm{f}}\right.$ in normalized units) must be several times greater than $\mathrm{p}_{\mathrm{o}}{ }^{2}$. Then the beam size at the magnet is roughly $\mathrm{x}_{\mathrm{f}} / \mathrm{p}_{\mathrm{o}}$; it is easily shown that such a beam does not change significantly in radius over a distance $\mathrm{x}_{\mathrm{f}}$ when the slope exiting the magnet is zero. Thus we can treat this beam in the presence of ions as if it were a beam with $p_{o, e f f e c t i v e}=x_{\mathrm{f}} / \mathrm{p}_{\mathrm{o}}$ on the target; the perturbed minimum will emerge from the target plane according to the results in section 5.4.2, using the effective spot size (and ignoring the last term for simplicity):

$$
x_{\text {iw, emerge }}=\frac{x_{f}}{p_{o}}\left(1+\frac{1}{\sqrt{2\left(\ln x_{f}^{2} / p_{o}^{2}-1\right)}}\right)
$$

Thus, this is the largest ion channel for which a time-varying magnet can compensate in this limit. One can try to concoct schemes where the nominal tune enters the magnet with positive slope, allowing a larger swing, but there are practical limits. Entering at zero slope and ramping the magnet from nominal to zero in the time for the ions to form the maximum allowed channel implies:

$$
\begin{aligned}
& v_{i} \Delta t=z_{i w} \approx L_{f} / p_{o} \\
& \dot{B} \approx \frac{B_{o}}{\Delta t}=\frac{B_{o} v_{i} p_{o}}{L_{f}}
\end{aligned}
$$

For $300 \mathrm{kV}$ hydrogen ions, a $20 \mathrm{~cm}$ focal length, and an $0.1 \mathrm{~T}$ focusing field, this would give a time variation of $0.04 \mathrm{~T} / \mathrm{ns}$ ( or 400 gauss $/ \mathrm{ns}$ ) for $\mathrm{p}_{\mathrm{o}}=10.0$, with control maintained for just $2.5 \mathrm{~ns}$. This is an impractical value.

For completeness, we give an approximate form for the time variation before the maximum channel size is exceeded. From section three, the minimum unperturbed spot size expressed as a function of the conditions at the magnet (the " $\mathrm{m}$ " subscript) is, in normalized form:

$$
p_{o, \text { effective }}^{2}=\frac{p_{m}^{2}}{1+p_{m}^{2} p_{m}^{\prime 2}}
$$

We will use this in the emergence condition but approximate the value of $p_{o, \text { effective }}$ in the $\log$ term with the maximum value $\left(\mathrm{p}_{\mathrm{m}}=\mathrm{x}_{\mathrm{f}} / \mathrm{p}_{\mathrm{o}}\right)$, since we know the magnet will vary so as to reduce the slope to zero: 


$$
x_{i w}=p_{o, \text { effective }}\left(1+\frac{1}{\sqrt{2\left(\ln x_{f}^{2} / p_{o}^{2}-1\right)}}\right)
$$

Solving for the slope, we find:

$$
\begin{aligned}
& p_{m}^{\prime}=\frac{p_{o}}{x_{f}} \sqrt{\frac{x_{f}^{2}\left(1+1 / \sqrt{2\left(\ln x_{f}^{2} / p_{o}^{2}-1\right)}\right)^{2}}{p_{o}^{2} x_{i w}^{2}}-1} \\
& R_{m}^{\prime}=R_{m, o}^{\prime} \frac{B^{2}}{B_{o}^{2}}=\frac{R_{o}}{L_{f}} \sqrt{\frac{L_{f}^{2}\left(1+1 / \sqrt{2\left(\ln K L_{f}^{2} / R_{o}^{2}-1\right)}\right)^{2}}{p_{o}^{2} v_{i}^{2} t^{2}}-1}
\end{aligned}
$$

Figure 7.2.2.1 shows a comparison of an uncontrolled beam to a controlled one (using equation (7.2.2.2)) for the case of $x_{f}=300.0$ and $p_{o}=10.0$. Figure 7.2.2.2 shows the controlled envelope all the way out to the magnet position, showing how the slope is reduced to zero at a maximum channel length of $\mathrm{x}_{\mathrm{iw}} \sim 39.0$. In terms of the required field, we note that $\mathrm{R}_{\mathrm{m}, \mathrm{o}}$, and $\mathrm{R}_{\mathrm{o}} / \mathrm{L}_{\mathrm{f}}$ cancel to good approximation, which expresses $\mathrm{B}(\mathrm{t})$ in terms of the fourth root of the argument of the radical. 

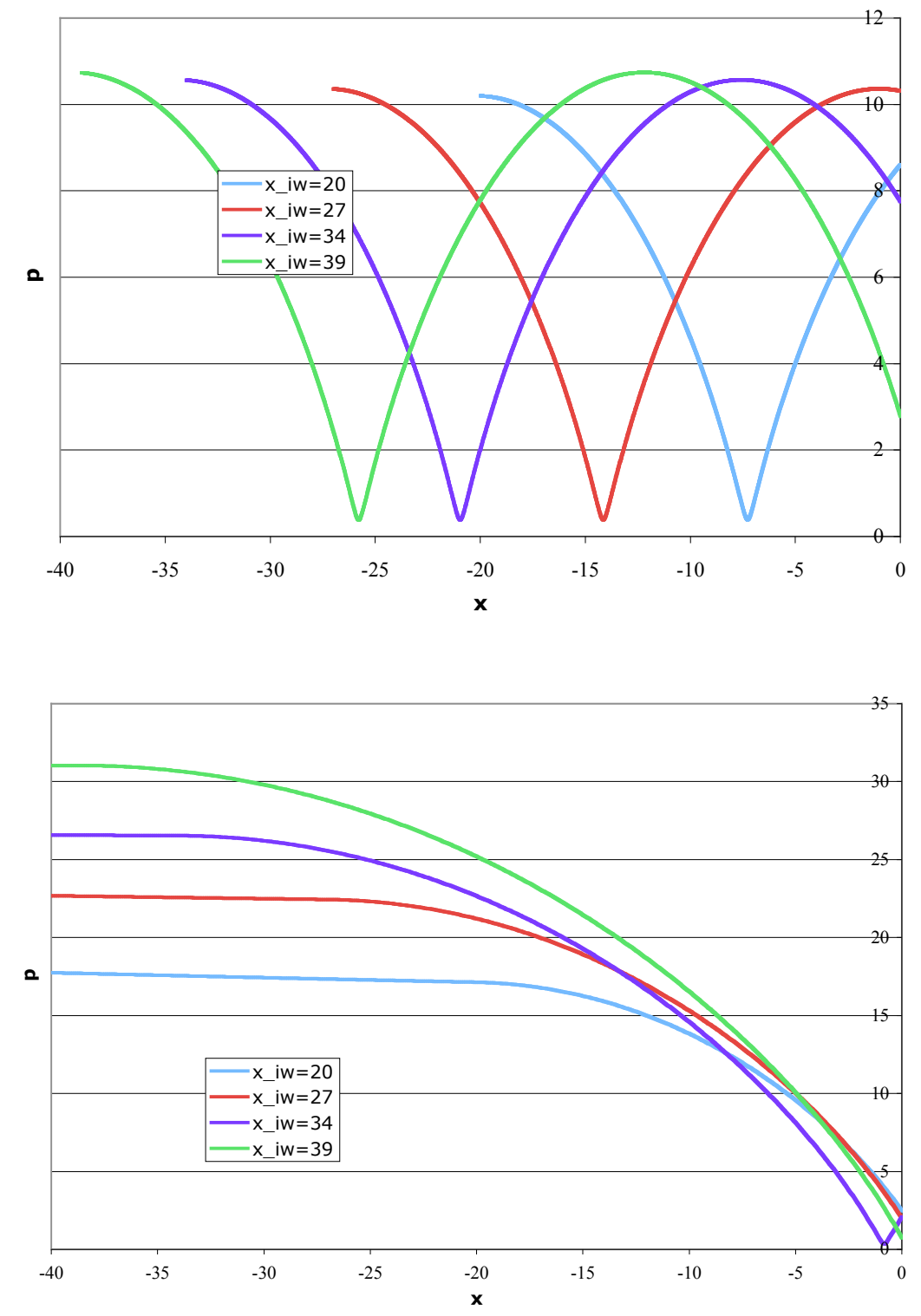

Figure 7.2.2.1. Comparison of uncontrolled spot growth as ion channel length increases (top) versus beam controlled via equation (7.2.2.2) (bottom), for $p_{0}=\mathbf{1 0 . 0}$. 


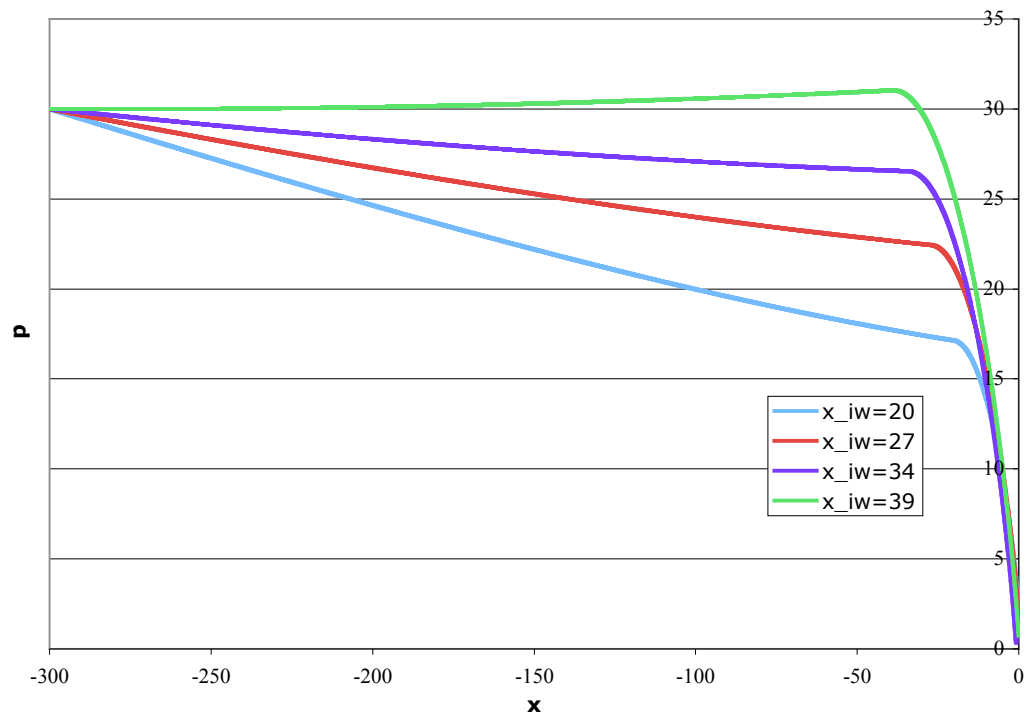

Figure 7.2.2.2. The controlled envelope, shown out to the position of a thin-lens final focus magnet. The field strength has been reduced to zero at the maximum controllable channel length.

\section{$\underline{7.3 \text { Energy Variation }}$}

Time variation in the energy of the beam will also affect the behavior in the presence of ions. The focusing provided by a thin-lens final focus magnet scales as $1 / \gamma^{2}$ and the focusing effect of the ions scales as $1 / \gamma$ (buried in the coefficient $\mathrm{K}$ as used in this report).

We will not go into any depth on quantifying this technique for beam control, but point out that the required analysis is nearly identical to that of section 7.2 except for the need to work in terms of $1 / \gamma$ rather than $B$, and to carefully account for the hidden dependence in normalized quantities such as $\mathrm{p}$ (with $1 / \sqrt{ } \gamma$ dependence) and $\mathrm{p}$ ' (with $1 / \gamma$ dependence) as well as the dependence in $\mathrm{K}$.

For $\mathrm{p}_{\mathrm{o}}<<1$, the bottom line comes quickest by letting $\mathrm{K}=\mathrm{K}_{\mathrm{o}} \gamma_{\mathrm{o}} / \gamma$ in (7.2.1.3) and replacing (7.2.1.4) by $\Delta \mathrm{R}^{\prime}=-2 \mathrm{R}_{\mathrm{o}}{ }^{\prime} \Delta \gamma / \gamma_{0}$, arriving at the energy-based equivalent of (7.2.1.5):

$$
\frac{\Delta \gamma}{\gamma_{o}}=\frac{p_{o}^{2} v_{i} t / 2 L_{f}}{1+p_{o}^{2} v_{i} t / 4 L_{f}}
$$

Treating the beam energy $\mathrm{E}$ as approximately proportional to $\gamma$, we find that for a $20 \mathrm{~cm}$ focal length, $p_{o}=0.1$, and $E_{o}=10 \mathrm{MV}$, an energy variation of about $2 \mathrm{kV} / \mathrm{ns}$ is required.

For $\mathrm{p}_{\mathrm{o}}>>1$, the problem is rather more difficult; instead of reducing the final focus field to zero, one must increase the beam energy to infinity! The desired waveform for this activity can be found, to leading order, by substituting $\gamma_{\mathrm{o}} / \gamma$ for $\mathrm{B} / \mathrm{B}_{\mathrm{o}}$ in (7.2.2.2) and approximating $\mathrm{K}$ with $\mathrm{K}_{\mathrm{o}}$ in the logarithm on the right-hand side. 


\subsection{Time-Averaged Focusing}

Suppose it is possible for the focusing system of the beam to produce a minimum spot size $R_{o}$ that is smaller than some acceptable operating size $R_{o k}$ (" $R$ okay"). Then it is possible to extend the length of the ion channel that can be tolerated by deliberately under-focusing the beam to some size $\mathrm{R}_{\text {start }}$ greater than $\mathrm{R}_{\mathrm{ok}}$ at the target plane, projecting the actual minimum $\mathrm{R}_{\mathrm{o}}$ some distance beyond, and achieving an time-averaged RMS size of $R_{\text {ok }}$ over the duration of the beam pulse as the minimum point moves from downstream to upstream of the target. We assume that $R_{\text {start }}$ is large enough compared to $R_{0}$ that the beam envelope has roughly constant slope near $R_{\text {start }}$ and that the approximate distance between the points where $R=R_{\text {start }}$ and $R=R_{0}$ is $d=R_{0} R_{\text {start }} / \varepsilon$. Assuming that the time over which the emittance-dominated beam traverses the target plane is small compared to the time it takes to vary linearly from the original under-focused sized, a short calculation of the time average of $\mathrm{R}^{2}(\mathrm{t})$ shows that the starting (and finishing) beam size is of order $R_{\text {start }} \sim \sqrt{3} R_{\text {ok }}$ to achieve a time average RMS size of $R_{0 k}$.

In the following subsections, note that $p_{o}$ is still defined in terms of $R_{o}$, not $R_{o k}$.

\subsection{1. $\mathrm{p}_{0}<<1$}

In this limit, we know that the perturbed waist size of the beam remains of order $p_{o}$ for very long channel lengths; in other words, the leading-order effect of the ion channel is simply to move the waist. Thus, with help from the diagrams shown in figure 7.4.1.1, we see that having a beam start and finish at a size $\sqrt{3} \mathrm{R}_{\mathrm{ok}}$ on the physical target plane corresponds to moving the perturbed waist a distance $2 \mathrm{~d}$ from original location of the unperturbed waist. Using the latter location as the origin for an effective channel length in equation (5.5.2.2), we find:

$$
\begin{aligned}
& \left|x_{m i n}-x_{t, e f f}\right|=x_{i w, e f f} p_{o}^{2}=2 K d / \epsilon=2 \sqrt{3} K R_{o} R_{o k} / \epsilon^{2} \\
& x_{i w, e f f}=2 \sqrt{3} p_{o k} / p_{o} \\
& x_{i w}=x_{i w, e f f}-2 K d / \epsilon \approx x_{i w, e f f}=2 \sqrt{3} p_{o k} / p_{o}
\end{aligned}
$$

The latter expression can be compared to the condition $\mathrm{x}_{\mathrm{iw}} \sim 1$ for the tolerable length if the beam starts at a waist on the target of size $\mathrm{R}_{0}$; it represents a noticeable improvement. In dimensional units,

$$
v_{i} t=2 \sqrt{3} \frac{\epsilon}{K} \frac{R_{o k}}{R_{o}}=2 \sqrt{\frac{3}{K}} \frac{R_{o k}}{p_{o}}
$$



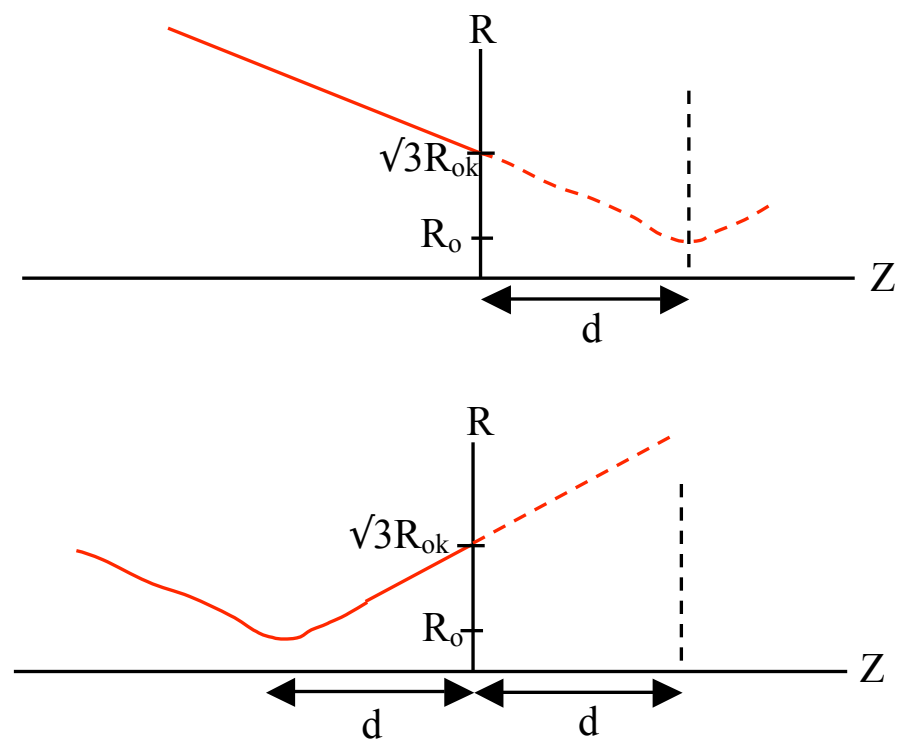

Figure 7.4.1.1. Conceptual picture of time-averaged focusing scheme, showing the starting (top) and ending (bottom) condition of the envelope relative to the target plane (solid vertical line).

\subsection{2. $\mathrm{p}_{\mathrm{o}} \gg 1$}

This limit requires a slightly different conceptual picture and produces a less favorable result. In order for the under-focused beam size $\sqrt{3} R_{0 k}$ to be reasonably greater than $R_{0}$, it implies that the normalized distance between the target plane and the location of the unperturbed waist (at some phantom point downstream of the target) be reasonably greater than $\mathrm{p}_{\mathrm{o}}{ }^{2}-$ i.e., the effective channel length $\mathrm{x}_{\mathrm{iw}, \text { eff }}$ is already in the long-channel regime. Hence, in the presence of an ion channel, the beam will already be "frozen" into multiple bounces according to the results of section 5.5; see figure 7.4.2.1 for an illustration. (Note that in this case the envelope on either side of the minima is clearly not linear, so the factor $\sqrt{ } 3$ converting $R_{o k}$ to $R_{\text {start }}$ is no longer correct; but we will keep it as a notional scaling). 


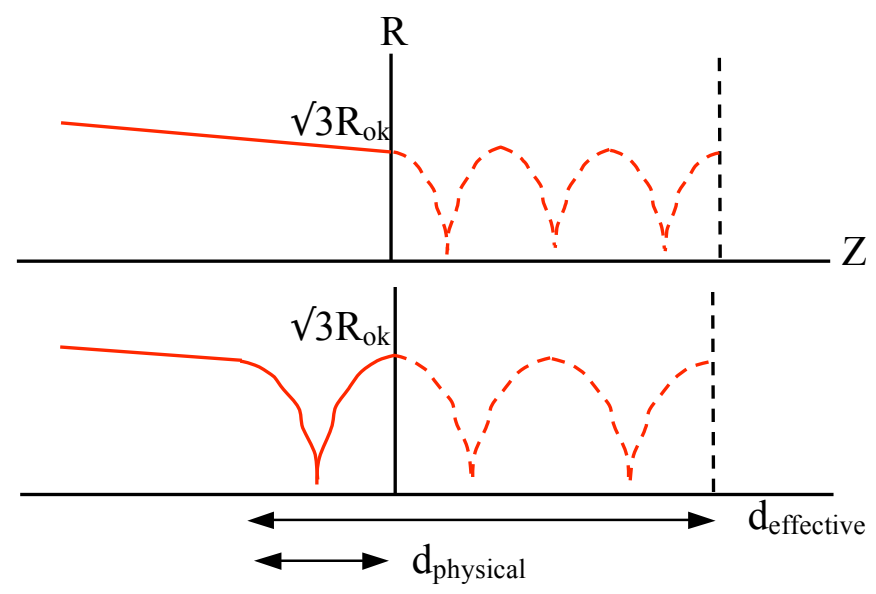

Figure 7.4.2.1. Conceptual picture of the po $>>1$ case, showing the starting (top) and ending (bottom) condition; the "phantom" channel beyond the target plane is already in the frozen-phase long channel regime.

In reality, it would not be desirable to bounce the beam multiple times (it is certainly questionable about whether a beam in this limit should be bounced even once). Thus the physical channel length that returns the beam to $\sqrt{3} R_{\mathrm{ok}}$ is (to leading order) a single wavelength, which from equation (5.6.1.1) gives:

$$
x_{i w} \approx \lambda=2 \frac{x_{i w, e f f}}{p_{o}}
$$

and in turn,

$$
x_{i w, e f f}=x_{i w}+K d / \epsilon=x_{i w}+\sqrt{3} p_{o k} p_{o}
$$

which gives to leading order:

$$
\begin{aligned}
& x_{i w}=2 \sqrt{3} p_{o k} \\
& v_{i} t=2 \sqrt{\frac{3}{K}} R_{o k}
\end{aligned}
$$

Thus, the ability of the focusing system to reach $p_{o}$ in principle is of no help; equation (7.4.2.1) is merely the disruption length corresponding to a time-averaged spot size of $\mathrm{R}_{\mathrm{ok}}$.

For completeness, we note that there is a time-averaging scheme which provides an advantage if it were possible for the beam to survive many strong bounces. One could time-average over the multiple bounces until the peak of the oscillations $\left(p_{\max }\right)$ exceeded 
$\sqrt{3} p_{\text {ok }}$. In the $\mathrm{p}_{\mathrm{o}}>>1$ limit, $\mathrm{p}_{\mathrm{max}} \sim \mathrm{p}_{\mathrm{i}}$ which leads to $\mathrm{x}_{\mathrm{iw}} \sim \sqrt{3} \mathrm{p}_{\mathrm{o}} \mathrm{p}_{\mathrm{ok}}$ or $\mathrm{v}_{\mathrm{i}} \mathrm{t} \sim(3 / \mathrm{K})^{1 / 2} \mathrm{p}_{\mathrm{o}} \mathrm{R}_{\mathrm{ok}}$, which is a substantial improvement.

\subsection{Channel Matching}

The final spot control option we discuss here is of a less practical nature, but is an interesting oddity that we include simply because it is counterintuitive: increasing the focusing strength of a magnet to offset the increased focusing of the ions!

This is possible in the one case we have not discussed much in this report: $p_{o}=1$. If $p_{o}=$ 1 is an acceptable spot size, then one can control the spot by maintaining $p_{i}=1, p_{i}{ }^{\prime}=0$ at the ion channel entrance. A possible two-magnet configuration for doing this is shown in the following figure:

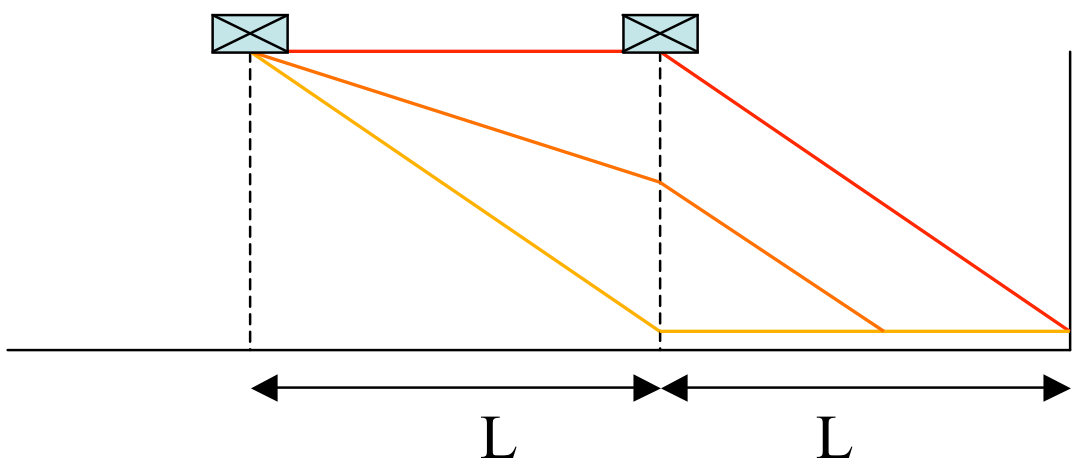

Figure 7.5.1. Schematic of $p_{0}=1$ channel matching, where the beam waist is kept at the entrance to the ion channel as a function of time. Only the upstream magnet varies.

In the configuration shown, only the upstream magnet varies. In this case, the only real ion physics that enters into the requirements is the ion speed; the beam waist must be maintained at the entrance to the channel at all times, until the channel grows large enough to reach the first magnet. (If the focusing provided by the downstream magnet is small enough for a beam of size $\mathrm{R}=\mathrm{R}_{\mathrm{eq}}$, it may be possible to accept the resulting small oscillations and have the scheme continue until the channel grows to the point of the shortest focal length provided by the upstream magnet.) Thus:

$$
R^{\prime}(t) \approx \frac{\epsilon v_{i} t}{R_{e q} L}=\sqrt{K} \frac{v_{i} t}{L}
$$

From the figure, it is clear that the peak field strength of the upstream magnet must be equal to the field strength (denoted $\mathrm{B}_{\mathrm{o}}$ ) required by the downstream magnet to put the unperturbed waist on the target, i.e., $\mathrm{B}(\mathrm{t})=\mathrm{B}_{\mathrm{o}} \mathrm{v}_{\mathrm{i}} \mathrm{t} / \mathrm{L}$. Using our benchmark quantities of $300 \mathrm{kV}$ hydrogen ions, a $20 \mathrm{~cm}$ focal length, and a nominal $0.1 \mathrm{~T}$ field, this works out to a magnet sweep rate of about 40 gauss/ns, with control maintained for $25 \mathrm{~ns}$. 


\section{Summary of Major Results}

The equilibrium radius $R_{\mathrm{eq}}$ is defined by $\varepsilon / \sqrt{\mathrm{K}}$, where $\mathrm{K}=\mathrm{fI} / \gamma \mathrm{I}_{\mathrm{A}}$ is the magnitude of the ion focusing force under the assumptions stated in section 3 . We use $R_{\text {eq }}$ to define a normalized beam radius $p=R / R_{\text {eq }}$ and distance $x=z \sqrt{K} / R_{\text {eq. }}$. The unperturbed size of the beam waist is denoted $p_{0}$. The ion channel length, measured from the upstream end to the location of the unperturbed beam waist, is denoted in normalized form as $\mathrm{x}_{\mathrm{iw}}$ and is timedependent. In terms of these quantities, here is a summary of some of the more useful results:

\begin{tabular}{|l|l|l|l|}
\hline & Section & $\mathbf{p}_{\mathbf{0}}<<\mathbf{1}$ & $\mathbf{p}_{\mathbf{0}}>>\mathbf{1}$ \\
\hline $\begin{array}{l}\text { Normalized } \\
\text { wavelength, } \lambda\end{array}$ & 5.6 & $\sqrt{2 \pi} \frac{x_{i w}}{p_{o}} e^{1 / 2 p_{o}^{2}}$ & $2 \frac{x_{i w}}{p_{o}}\left(1+\frac{1}{\sqrt{2\left(\ln x_{i w}^{2} / p_{o}^{2}-1\right)}}\right)$ \\
\hline $\begin{array}{l}\text { Disruption } \\
\text { length (strict), } \\
\mathbf{x}_{\mathbf{D}}\end{array}$ & 5.5 & $p_{o} \sqrt{\ln 1 / p_{o}^{2}}$ & $2 p_{o}\left(1+\frac{1}{\left.\sqrt{2\left(\ln p_{o}^{2}-1\right)}-\frac{1+\sqrt{2}}{2 p_{o}}\right)}\right.$ \\
\hline $\begin{array}{l}\text { Disruption } \\
\text { length } \\
\text { (effective) }\end{array}$ & 5.6 & $x_{D, e f f}=1$ & $x_{D, \text { eff }}=x_{D}$ \\
\hline $\begin{array}{l}\text { Disruption } \\
\text { rate, ptarg(t) }\end{array}$ & 5.6 & $p_{o} \sqrt{1+x_{i w}^{2}}$ & $x_{i w} / p_{o}$, envelope of oscillations \\
\hline $\begin{array}{l}\text { Number of } \\
\text { oscillations in } \\
\text { "frozen” } \\
\text { regime }\end{array}$ & 5.6 & Never bounces & $p_{o} / 2$ \\
\hline $\begin{array}{l}\text { Slope sweep } \\
\text { rate for spot } \\
\text { control }\end{array}$ & 7.2 & $\Delta R^{\prime} \approx-\frac{p_{o} v_{i} t \sqrt{K}}{L_{f}}$ & $R^{\prime}=\frac{R_{o}}{L_{f}} \sqrt{\frac{L_{f}^{2}\left(1+1 / \sqrt{\left.2\left(\ln K L_{f}^{2} / R_{o}^{2}-1\right)\right)^{2}}\right.}{p_{o}^{2}}-1}$ \\
\hline $\begin{array}{l}\text { Field sweep } \\
\text { rate for spot } \\
\text { control }\end{array}$ & 7.2 & $\Delta B=-\frac{B_{o}}{2} \frac{v_{i} t}{L_{f}} p_{o}^{2}$ & $\Delta B=-\frac{B_{o} v_{i} p_{o}}{L_{f}}$ \\
\hline $\begin{array}{l}\text { Tolerable } \\
\text { channel length } \\
\text { using time- } \\
\text { averaged } \\
\text { focusing }\end{array}$ & 7.4 & $v_{i} t=2 \sqrt{\frac{3}{K}} \frac{R_{o k}}{p_{o}}$ & $v_{i} t=2 \sqrt{\frac{3}{K} R_{o k}}, v_{i} t=\sqrt{\frac{3}{K}} p_{o} R_{o k}$, one bounce; \\
\hline
\end{tabular}

For the spot-control options, $\mathrm{L}_{\mathrm{f}}$ is the focal length of a thin-lens final focus magnet set a field strength of $B_{o}$ in unperturbed conditions, and $R_{o k}$ denotes a time-averaged spot size that is acceptable even though the focusing system may be capable of producing a smaller size $\mathrm{R}_{\mathrm{o}}$ (which then determines $\mathrm{p}_{\mathrm{o}}$ ).

In general, the $\mathrm{p}_{\mathrm{o}}<<1$ regime is much less sensitive to ion disruption. 


\section{References}

[1]. Nezlin, M. V. Physics of Intense Beams in Plasmas. IOP Publishing, Philadelphia, PA, 1993.

[2]. Burns, M. et al. "Overview and Status of the Dual-Axis Radiographic Hydrodynamics Test (DARHT) Facility," $25^{\text {th }}$ International Power Modulator Symposium, IEEE 2002, pp. 97-101.

[3]. Sampayan, S. et al. "Beam-Target Interaction Experiments for Multipulse Bremsstrahlung Converter Applications." Proceedings of the 2001 Particle Accelerator Conference. IEEE vol. 1, 2002, pp 330-2.

[4]. Hughes, T. P. et al. "Electron Beam Disruption due to Ion Release from Target Comparison Between Computer Calculations and Observations," 2002 IEEE International Conference on Plasma Science, IEEE 2002, pp. 251.

[5]. Caporaso, G. J. and Chen, Y.-J. "Analytic Model of Ion Emission from the Focus of an Intense Relativistic Electron Beam on a Target," Proceedings of the $19^{\text {th }}$ International Linear Accelerator Conference (LINAC), Chicago Il, 1998, vol. 2 pp. 830-2.

[6]. Chen, Y.-J., Caporaso, G. J., Paul, A. C. “Controlling Backstreaming Ions from XRay Converter Targets with Time-Varying Final Focusing Solenoidal Lens and Beam Energy Variation," Proceedings of the $19^{\text {th }}$ International Linear Accelerator Conference (LINAC), Chicago Il, 1998, vol. 1 pp. 472-4.

[7]. Lee, E. P. and Cooper, R. K. "General Envelope Equation for Cylindrically Symmetric Charged Particle Beams, “Particle Accelerators, 1976, vol. 7, pp. 83-95.

[8]. Humphries, S. Charged Particle Beams. John Wiley \& Sons, New York, 1990. See chapter 5 .

[9]. McCarrick, J. F. "Electron Beam/Converter Target Interactions in Radiographic Accelerators," Proceedings of the 2003 Particle Accelerator Conference, IEEE, 2003, vol. 1, pp. 563-7.

[10]. Reiser, M. Theory and Design of Charged Particle Beams. John Wiley \& Sons, New York, 1994. See chapter 3. 


\section{Appendix A. Algebraic Details of the Analytic Solutions}

In this Appendix, we present the details of the algebra used to find approximate solutions of equation (5.1.1). The steps include setup of the quadrature form and then different solution techniques for two different regions of the envelope. The last section compares the analytic solution with numerical calculations.

\section{A.1. Setup of the Quadrature Form}

Let us begin with the normalized form of the full equation (retaining both emittance and ion focusing):

$$
p^{\prime \prime}=\frac{1}{p^{3}}-\frac{1}{p}
$$

We reduce the order of the equation by taking advantage of the autonomous form (no explicit dependence on $\mathrm{x}$ ). Multiplying though by $\mathrm{dp} / \mathrm{dx}$ and integrating gives:

$$
p^{2}-p_{i}^{2}=\frac{1}{p_{i}^{2}}-\frac{1}{p^{2}}-\ln p^{2} / p_{i}^{2}
$$

where $\mathrm{p}_{\mathrm{i}}$ and $\mathrm{p}_{\mathrm{i}}$ are the conditions at the upstream entrance of the ion channel. Define:

$$
C \equiv p_{i}^{\prime 2}+\ln p_{i}^{2}+\frac{1}{p_{i}^{2}}
$$

and re-write the equation in quadrature form after solving for $\mathrm{dp} / \mathrm{dx}$ :

$$
\int_{p_{i}}^{p} \frac{d p}{\sqrt{C-\ln p^{2}-1 / p^{2}}}= \pm\left(x-x_{i}\right)
$$

The location of the channel entrance is denoted by $\mathrm{x}_{\mathrm{i}}$. The integrand has integrable singularities at two points, denoted by $\mathrm{p}_{\min }$ and $\mathrm{p}_{\max }$, corresponding to minima and maxima of oscillations in the beam envelope. By definition, $p_{\max } \geq 1$ and $p_{\min } \leq 1$, with equality only possible for perfectly match transport $\left(\mathrm{p}_{\mathrm{i}}=1, \mathrm{p}_{\mathrm{i}}{ }_{\mathrm{i}}=0\right)$. They can be found without solving for the entire envelope by using the following recursions:

$$
\begin{aligned}
& p_{\text {max }}^{2}=e^{C-1 / p_{\text {max }}^{2}} \\
& p_{\text {min }}^{2}=\frac{1}{C-\ln p_{\text {min }}^{2}}
\end{aligned}
$$

The integral does not have a simple closed form, but we can approximate it in piecewise fashion. The main requirement for the process is that the resulting expression for $\mathrm{x}(\mathrm{p})$ must be invertible to $\mathrm{p}(\mathrm{x})$, which is much more of interest. 
For illustration, the positive branch of the (p,p') phase space

$$
p^{\prime}=-\sqrt{C-1 / p^{2}-\ln p^{2}}
$$

is shown in Figure A.1.1 for several values of C; note the log scale for p.

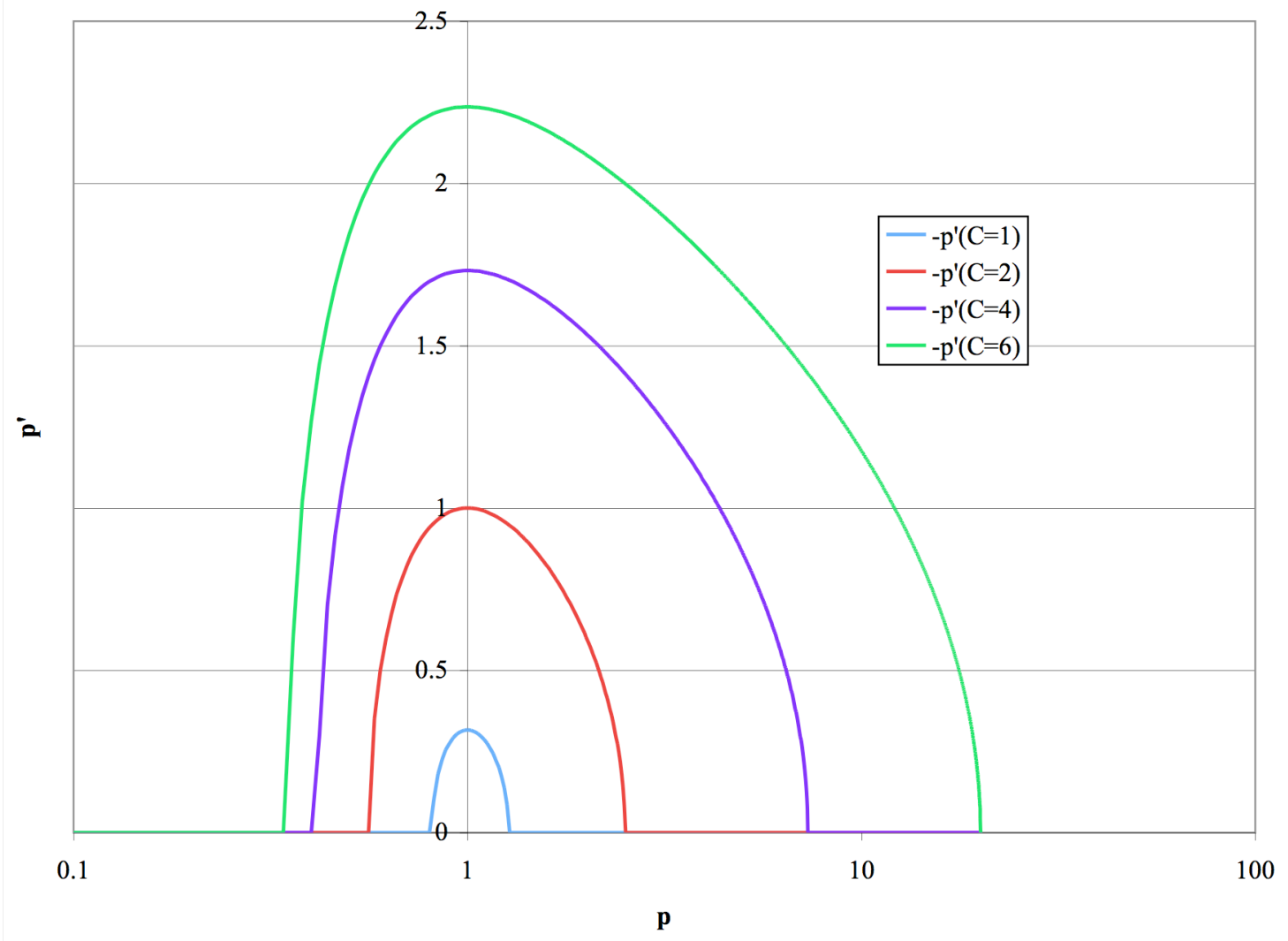

Figure A.1.1. Families of normalized slope versus radius, showing two roots. Note the log scale used for $p$.

\section{A.2. Solution in the Region $\underline{p}_{\min } \leq p \leq 1$}

In the region $\mathrm{p}_{\min } \leq \mathrm{p} \leq 1$, we do not explicitly remove the singularity at $\mathrm{p}_{\min }$, but instead invoke a curve fit which captures the position and slope correctly. A better fit is obtained by working in terms of $\mathrm{y}=\mathrm{p}^{2}$ :

$$
\frac{1}{2} \int_{p_{\min }^{2}}^{p^{2} \leq 1} \frac{d y}{\sqrt{C y-1-y \ln y}}=\left|x-x_{\min }\right|
$$

where $\mathrm{x}_{\min }$ denotes the location where $\mathrm{p}=\mathrm{p}_{\min }$. Let:

$$
C y-1-y \ln y \approx \alpha\left(p^{2}-p_{m i n}^{2}\right)\left(p_{e f f}^{2}-p^{2}\right)
$$


where we choose $\alpha$ and the effective maximum $\mathrm{p}_{\text {eff }}^{2}$ such that:

$$
\begin{aligned}
& \left.(C y-1-y \ln y)^{\prime}\right|_{y=p_{m i n}^{2}}=C-1-\ln p_{\text {min }}^{2}=\alpha\left(p_{e f f}^{2}-p_{\text {min }}^{2}\right) \\
& \left.(C y-1-y \ln y)\right|_{y=1}=C-1=\alpha\left(1-p_{\min }^{2}\right)\left(p_{e f f}^{2}-1\right)
\end{aligned}
$$

which solve to:

$$
\begin{aligned}
& p_{e f f}^{2}=\frac{\left(1-p_{\min }^{2}\right)^{2}-p_{\min }^{4}(C-1)}{\left(1-p_{\min }^{2}\right)^{2}-p_{\min }^{2}(C-1)} \\
& \alpha=\frac{1 / p_{\min }^{2}-1}{p_{e f f}^{2}-p_{\min }^{2}}
\end{aligned}
$$

The function and fit are shown below. Note that we have explicitly matched the asymptotic behavior near the zero (which, in the integrand, becomes a singularity); without doing so, a fit that "looks good" visually would not actually have much relevance.

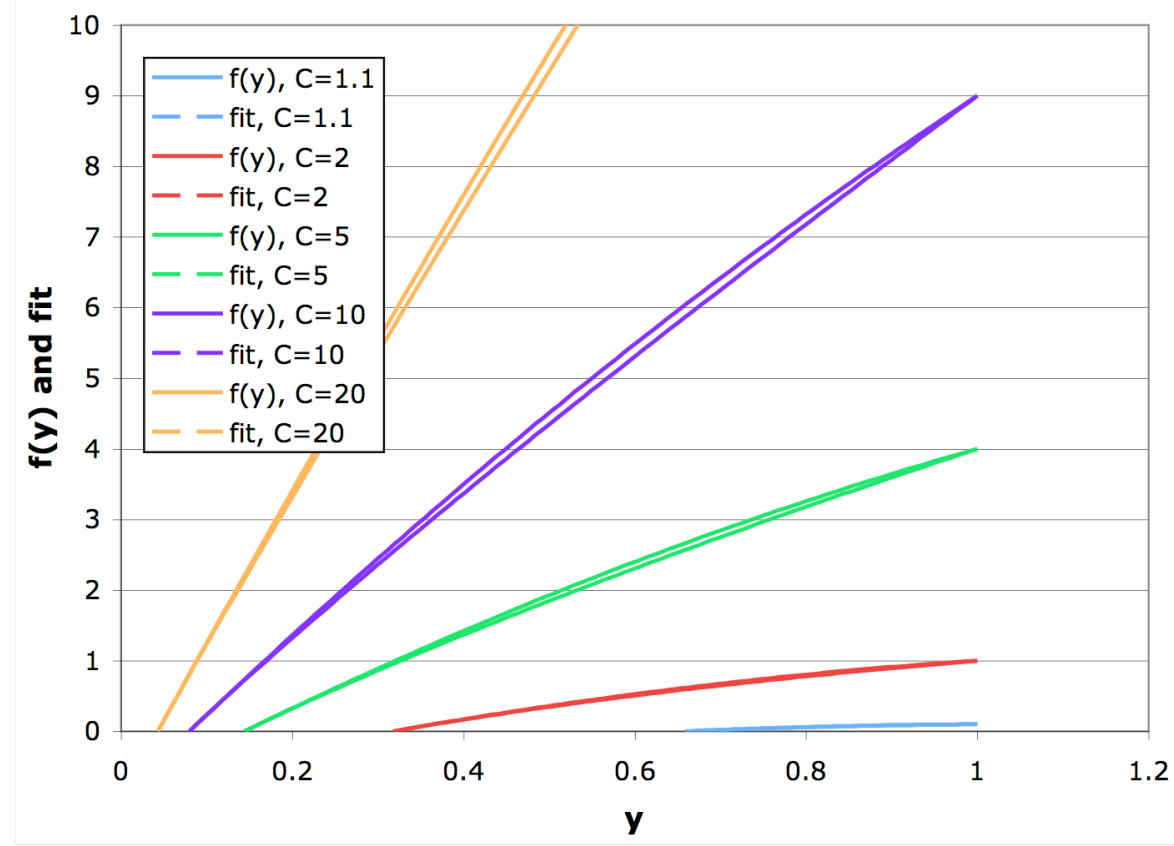

Figure A.2.1. Replacement of a function in the integrand with an analytically tractable form, in the region $\mathrm{p}_{\text {min }}^{2}<y=\mathrm{p}^{2}<1$.

The integral can now be approximated:

$$
\left|x-x_{m i n}\right| \approx \frac{1}{2 \sqrt{\alpha}} \int_{p_{\min }^{2}}^{p^{2} \leq 1} \frac{d y}{\sqrt{\left(y-p_{\min }^{2}\right)\left(p_{e f f}^{2}-y\right)}}
$$




$$
\begin{gathered}
=-\left.\frac{1}{2 \sqrt{\alpha}} \sin ^{-1}\left(\frac{p_{e f f}^{2}+p_{\min }^{2}-2 y}{\sqrt{\left(p_{e f f}^{2}+p_{\min }^{2}\right)^{2}-4 p_{e f f}^{2} p_{\min }^{2}}}\right)\right|_{p_{\text {min }}^{2}} ^{p^{2}} \\
=-\frac{1}{2 \sqrt{\alpha}}\left[\sin ^{-1}\left(\frac{p_{e f f}^{2}+p_{\min }^{2}-2 p^{2}}{p_{e f f}^{2}-p_{\min }^{2}}\right)-\frac{\pi}{2}\right]=\frac{1}{2 \sqrt{\alpha}} \cos ^{-1}\left(\frac{p_{e f f}^{2}+p_{\min }^{2}-2 p^{2}}{p_{e f f}^{2}-p_{\min }^{2}}\right)
\end{gathered}
$$

And then inverted to give the envelope in the region where $\mathrm{p}^{2} \leq 1$ :

$$
p^{2}(x)=\frac{p_{e f f}^{2}+p_{m i n}^{2}}{2}-\frac{p_{e f f}^{2}-p_{m i n}^{2}}{2} \cos 2 \sqrt{\alpha}\left|x-x_{m i n}\right|
$$

The location of the minimum, $\mathrm{x}_{\min }$, can be found relative to known positions based on initial conditions. If $\mathrm{p}_{\mathrm{i}}^{2} \leq 1$ then:

$$
\left|x_{m i n}-x_{i}\right|=\frac{1}{2 \sqrt{\alpha}} \cos ^{-1}\left(\frac{p_{e f f}^{2}+p_{\text {min }}^{2}-2 p_{i}^{2}}{p_{e f f}^{2}-p_{\text {min }}^{2}}\right)
$$

Otherwise,

$$
\left|x_{m i n}-x_{1}\right|=\frac{1}{2 \sqrt{\alpha}} \cos ^{-1}\left(\frac{p_{e f f}^{2}+p_{m i n}^{2}-2}{p_{e f f}^{2}-p_{m i n}^{2}}\right)
$$

where $\mathrm{x}_{1}$ denotes the location where $\mathrm{p}=1$; it is found by solving for the envelope in the $p^{2}>1$ region, treated in the next section. The signs of the displacements relative to $x_{\min }$ are determined by whether the beam is converging or diverging.

\section{A.3. Solution in the Region $1 \leq p \leq \mathrm{p}_{\max }$}

In the region $1 \leqq \mathrm{p} \leqq \mathrm{p}_{\max }$, we need to remove both singularities to have a usable form; there are a number of options in general, but the choice that leaves a form that is both simple and invertible is to remove them sequentially, starting with the one at $\mathrm{p}_{\max }$. We start with the $y=p^{2}$ form used in section A.2 and remove the square-root singularity with another change of variable:

$$
\begin{aligned}
& s \equiv \sqrt{p_{\max }^{2}-y}, y=p_{\max }^{2}-s^{2}, d y=-2 s d s \\
& \left|x-x_{1}\right|=\frac{1}{2} \int_{s_{1}}^{\sqrt{p_{\text {max }}^{2}-p^{2}}} \frac{-2 s d s}{\sqrt{y(s)(C-\ln y(s))-1}}, s_{1} \equiv \sqrt{p_{\text {max }}^{2}-1}
\end{aligned}
$$

Now a similar change removes the singularity at the other end: 


$$
\begin{aligned}
& s_{\max } \equiv \sqrt{p_{\max }^{2}-p_{\min }^{2}}, t \equiv \sqrt{s_{\max }-s}, s=s_{\max }-t^{2}, d s=-2 t d t \\
& \left|x-x_{1}\right|=\int_{t_{1}}^{t} \frac{2 t s(t) d t}{\sqrt{y(t)(C-\ln y(t))-1}}, t_{1} \equiv \sqrt{s_{\max }-s_{1}}
\end{aligned}
$$

As $t$ approaches $t_{\max } \equiv \sqrt{s_{\max }}$,

$$
\begin{aligned}
& t \rightarrow t_{\max }-\epsilon \\
& s \rightarrow s_{\max }-\left(s_{\max }-2 \sqrt{s_{\max }} \epsilon\right) \rightarrow 2 t_{\max } \epsilon \\
& y \rightarrow p_{\max }^{2}-\left(2 t_{\max } \epsilon\right)^{2} \rightarrow p_{\max }^{2}\left(1-\frac{4 s_{\max } \epsilon^{2}}{p_{\max }^{2}}\right)
\end{aligned}
$$

In this limit, the integrand $\mathrm{I}(\mathrm{t})$ goes to:

$$
\begin{aligned}
I\left(t_{\max }\right) \equiv I_{\max } & \rightarrow \frac{2 t s}{\sqrt{y(C-\ln y)-1}} \rightarrow \frac{4 s_{\max } \epsilon}{\sqrt{p_{\max }^{2}\left(1-\frac{4 s_{\max } \epsilon^{2}}{p_{\max }^{2}}\right)\left(C-\ln p_{\max }^{2}+\frac{4 s_{\max } \epsilon^{2}}{p_{\max }^{2}}\right)-1}} \\
& \rightarrow \sqrt{\frac{4 s_{\max }}{1+\ln p_{\max }^{2}-C}} \rightarrow \sqrt{\frac{4 s_{\max }}{1-1 / p_{\max }^{2}}}
\end{aligned}
$$

Equation A.3.1 hardly seems to satisfy the given description of "simple." However, plots of the integrand for various values of $C$ in the range $t_{1}<t<t_{\max }$ (where $t_{1} \equiv t(p=1)$ ) shows that we have successfully transformed the integrand into something that is smooth everywhere and "boring" enough to be replaced by much more tractable functions: 


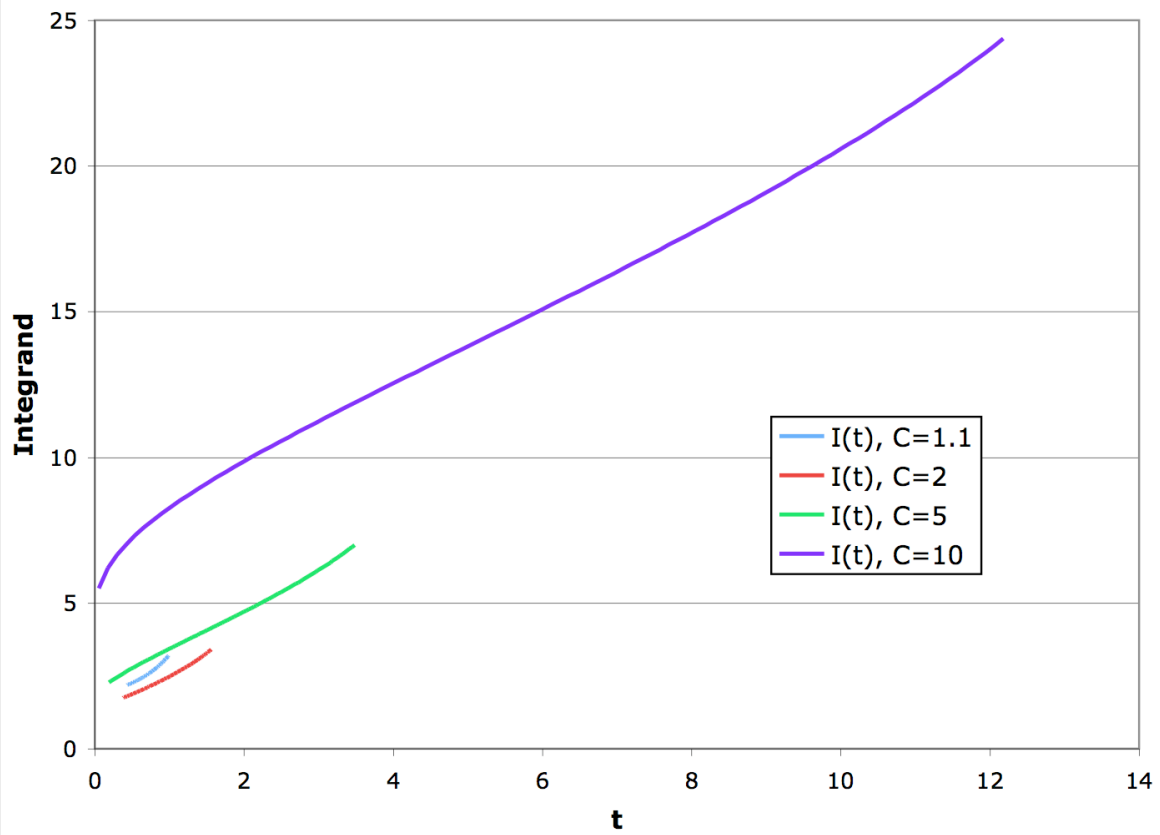

Figure A.3.1. Transformed integrand in the region $1<p(t)<p_{\max }$, for smaller values of C.

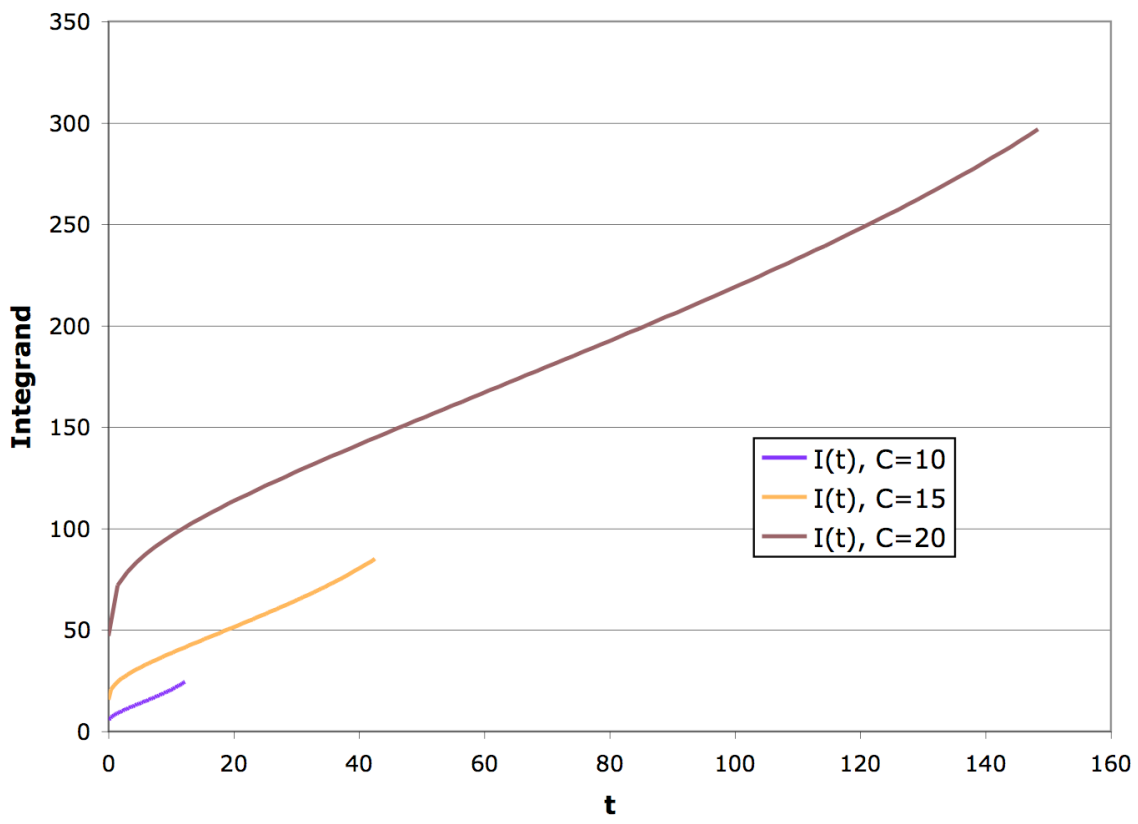

Figure A.3.2. Transformed integrand in the region $1<p(t)<p_{\max }$, for larger values of $\mathbf{C}$.

Defining $t_{i}=t\left(p=p_{i}\right), s_{i}=s\left(p=p_{i}\right)$, we identify two other useful values of $I(t)$ :

$$
I\left(t_{i}\right) \equiv I_{i}=\frac{2 t_{i} s_{i}}{\left|p_{i} p_{i}^{\prime}\right|}
$$




$$
I\left(t_{1}\right) \equiv I_{1}=\frac{2 t_{1} s_{1}}{\sqrt{C-1}}
$$

Using these, we approximate the integral in the simplest possible way: a straight line between the endpoints of integration. Again we assume the beam is initially converging. If $p_{i}>1$, we break the envelope into two pieces, on either side of $x_{i}$. For points between $x_{i}$ and $\mathrm{x}_{1}$,

$$
\left|x-x_{1}\right| \approx \int_{t_{1}}^{t}\left(I_{1}+\frac{I_{i}-I_{1}}{t_{i}-t_{1}}\left(t-t_{1}\right)\right) d t=I_{1}\left(t-t_{1}\right)+\frac{I_{i}-I_{1}}{t_{i}-t_{1}} \frac{\left(t-t_{1}\right)^{2}}{2}
$$

For points between $\mathrm{x}_{\mathrm{i}}$ and $\mathrm{x}_{\max }$,

$$
\left|x-x_{i}\right| \approx \int_{t_{i}}^{t}\left(I_{i}+\frac{I_{\max }-I_{i}}{t_{\max }-t_{i}}\left(t-t_{i}\right)\right) d t=I_{i}\left(t-t_{i}\right)+\frac{I_{\max }-I_{i}}{t_{\max }-t_{i}} \frac{\left(t-t_{i}\right)^{2}}{2}
$$

In practice, there are many cases where it will be more accurate to use the upper expression to extrapolate into the region between $\mathrm{x}_{\mathrm{i}}$ and $\mathrm{x}_{\max }$, since the scales $\left|\mathrm{x}_{\mathrm{i}}-\mathrm{x}_{\max }\right|$ and $\left|\mathrm{x}_{\mathrm{i}}-\mathrm{x}_{1}\right|$ can become quite different as the parameter $\mathrm{C}$ takes on values away from 1 . The two locations $\mathrm{x}_{1}$ and $\mathrm{x}_{\max }$ are found from:

$$
\begin{aligned}
& \left|x_{1}-x_{i}\right|=\frac{\left(I_{i}+I_{1}\right)\left(t_{i}-t_{1}\right)}{2} \\
& \left|x_{i}-x_{\max }\right|=\frac{\left(I_{\max }+I_{i}\right)\left(t_{\max }-t_{i}\right)}{2}
\end{aligned}
$$

For $\mathrm{p}_{\mathrm{i}}<1$, the only choice is extrapolation, using $\mathrm{x}_{1}$ as calculated from section A.2:

$$
\left|x-x_{1}\right| \approx \int_{t_{1}}^{t}\left(I_{1}+\frac{I_{\max }-I_{1}}{t_{\max }-t_{1}}\left(t-t_{1}\right)\right) d t=I_{1}\left(t-t_{1}\right)+\frac{I_{\max }-I_{1}}{t_{\max }-t_{1}} \frac{\left(t-t_{1}\right)^{2}}{2}
$$

and the distance between $\mathrm{x}_{1}$ and $\mathrm{x}_{\max }$ is given by:

$$
\left|x_{\max }-x_{1}\right|=\frac{\left(I_{\max }+I_{1}\right)\left(t_{\max }-t_{1}\right)}{2}
$$

As with the $p<1$ region, the relative signs of the offsets are determined by whether the beam is converging or diverging. Inverting expressions such as A.3.2 to calculate $\mathrm{p}(\mathrm{x})$ is messy but, by design, doable: 
$p^{2}(x)=p_{\text {max }}^{2}-\left(p_{\text {max }}^{2}-p_{\text {min }}^{2}\right)\left[1-\left[\frac{t_{1}}{t_{\text {max }}}-\frac{2\left(t_{i}-t_{1}\right) I_{1}}{\left(I_{i}-I_{1}\right) t_{\max }}+\sqrt{\frac{\left(t_{i}-t_{1}\right)^{2} I_{1}^{2}}{\left(I_{i}-I_{1}\right)^{2} t_{\text {max }}^{2}}+\frac{2\left(t_{i}-t_{1}\right)\left|x-x_{1}\right|}{\left(I_{i}-I_{1}\right) t_{\text {max }}^{2}}}\right]^{2}\right]^{2}$

The entire envelope can now be constructed by stepping through multiple wavelengths using the appropriate expressions for $\mathrm{p}$ less than or greater than 1. A complete oscillation spans $\lambda=2\left|\mathrm{x}_{\max }-\mathrm{x}_{\min }\right|$.

\section{A.4. Comparison with Numerical Solutions}

In this section we show comparisons of the approximate solutions given in section 3 to numerically integrated solutions. To cover a wide range of incoming beam conditions, we will work in terms of what will be called a nominal beam: an emittance-dominated envelope focused on an emission surface located at $z=0$, and focused (in the absence of ions) to a size $R_{0}$. The normalized form of equation (3.2) is then:

$$
p^{2}(x)=p_{o}^{2}+\frac{x^{2}}{p_{o}^{2}}
$$

with the slope given by:

$$
p^{2}(x)=\frac{x^{2} / p_{o}^{2}}{p_{o}^{4}+x^{2}}
$$

These relations provide a mapping between the incoming beam conditions $\left(\mathrm{p}_{\mathrm{i}}, \mathrm{p}_{\mathrm{i}}{ }^{\prime}\right)$ at the ion channel entrance and the parameters $\mathrm{p}_{\mathrm{o}}$ and $\mathrm{x}_{\mathrm{i}}$, the normalized unperturbed spot size and channel length, respectively:

$$
\begin{aligned}
& p_{i}^{2}=p_{o}^{2}+\frac{x_{i}^{2}}{p_{o}^{2}} \\
& p_{i}^{\prime 2}=\frac{x_{i}^{2} / p_{o}^{2}}{p_{o}^{4}+x_{i}^{2}}
\end{aligned}
$$

The analytic expressions are satisfactory except for cases where $\mathrm{p}_{\mathrm{o}}<1$ and $\mathrm{x}_{\mathrm{i}}>\mathrm{p}_{\mathrm{o}}$; in this limit, the absolute phase error caused by inaccuracy in the wavelength takes its toll on the predicted spot size on the target plane. It is worth noting that the relative error in the wavelength is still small.

Particular aspects of the behavior of the solution are discussed in the main text. 

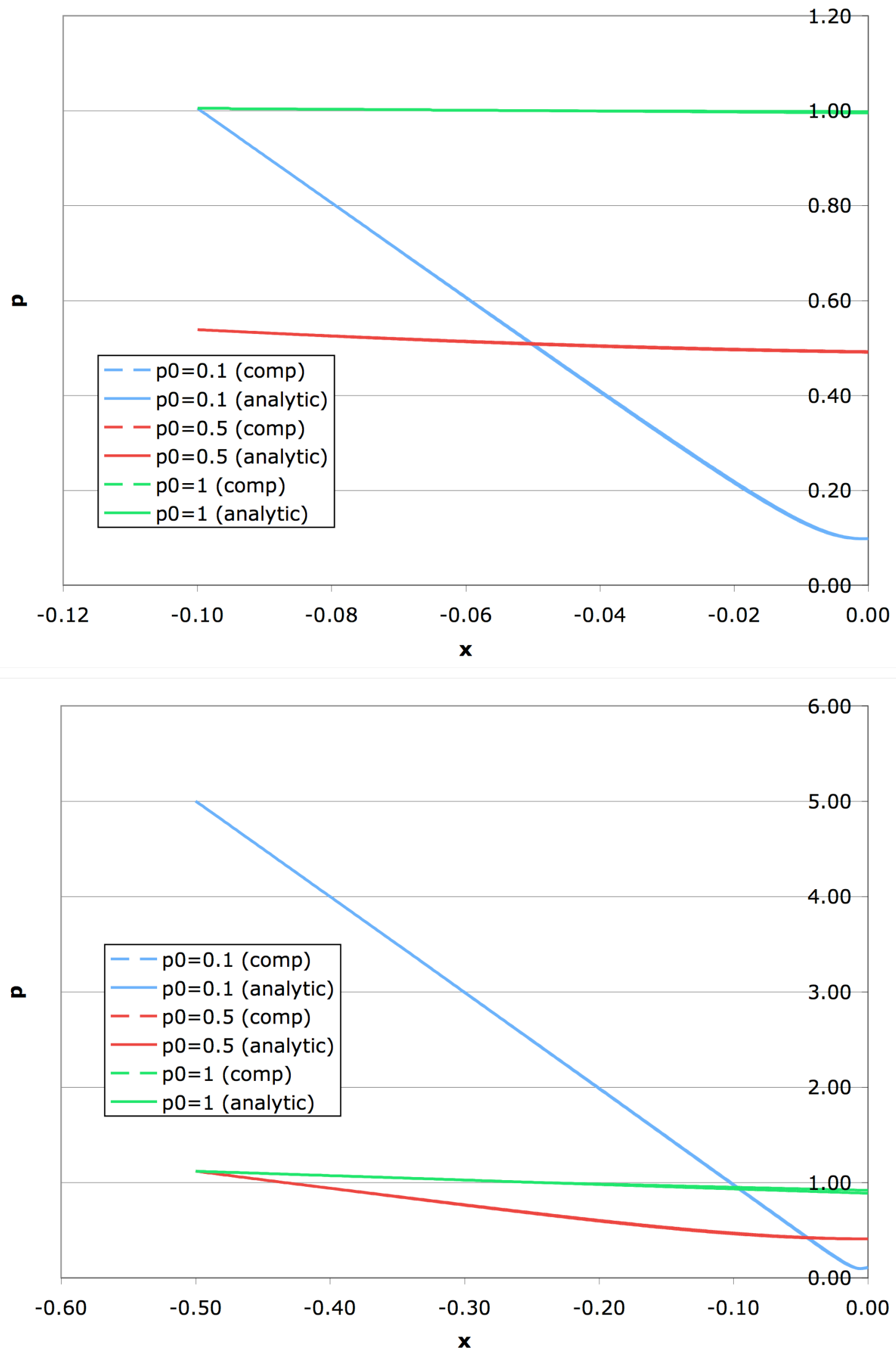

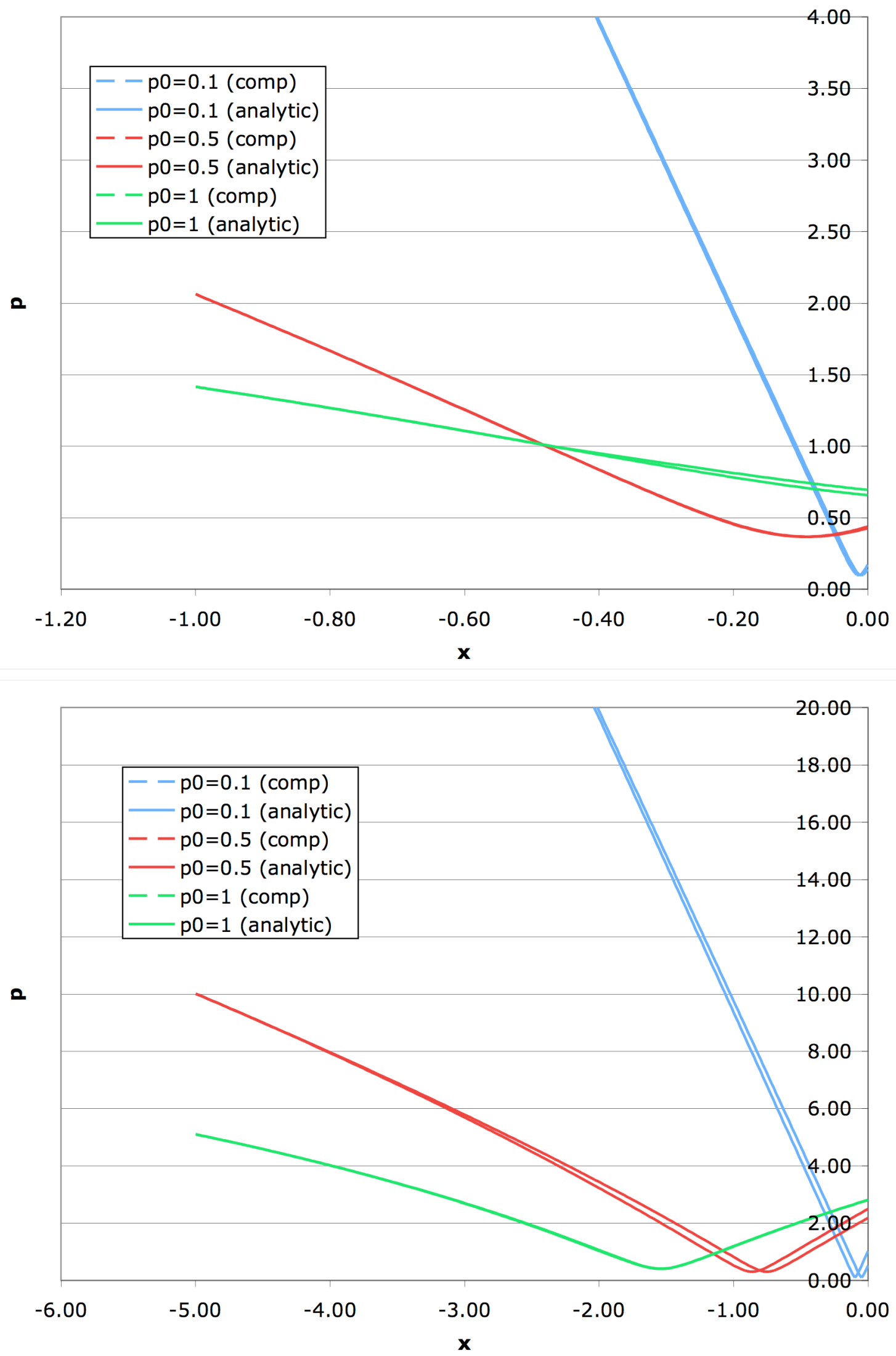

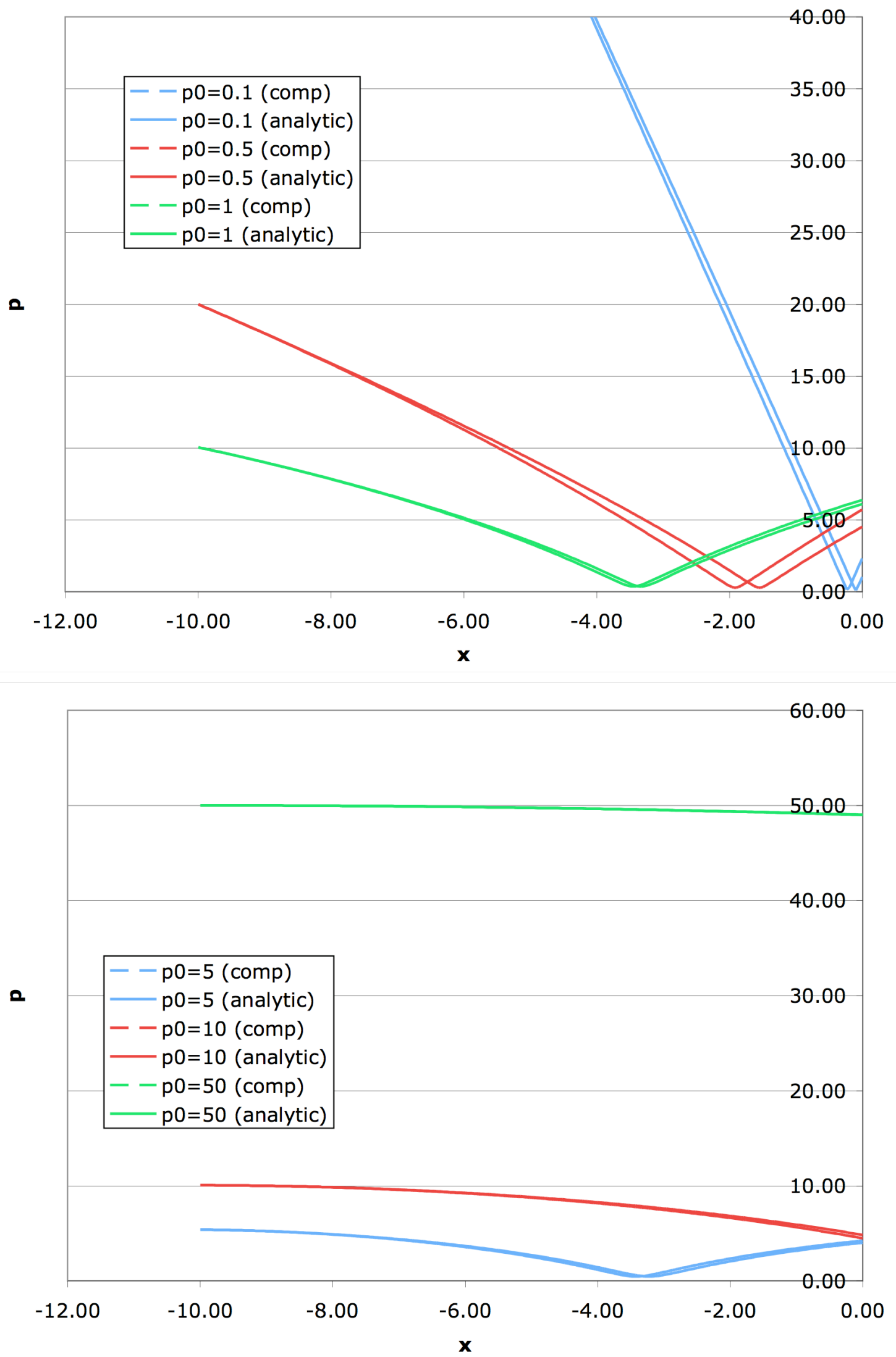

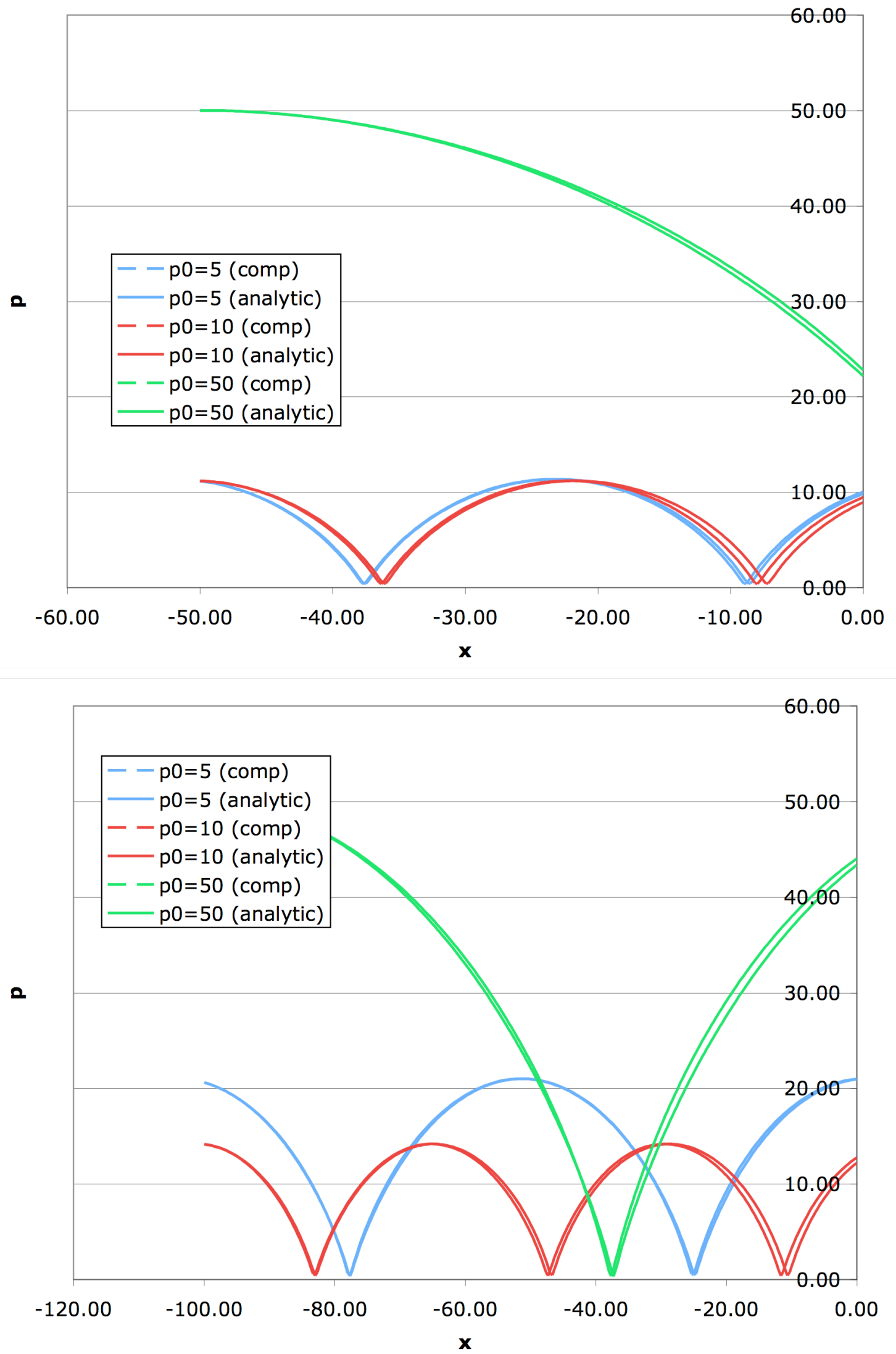

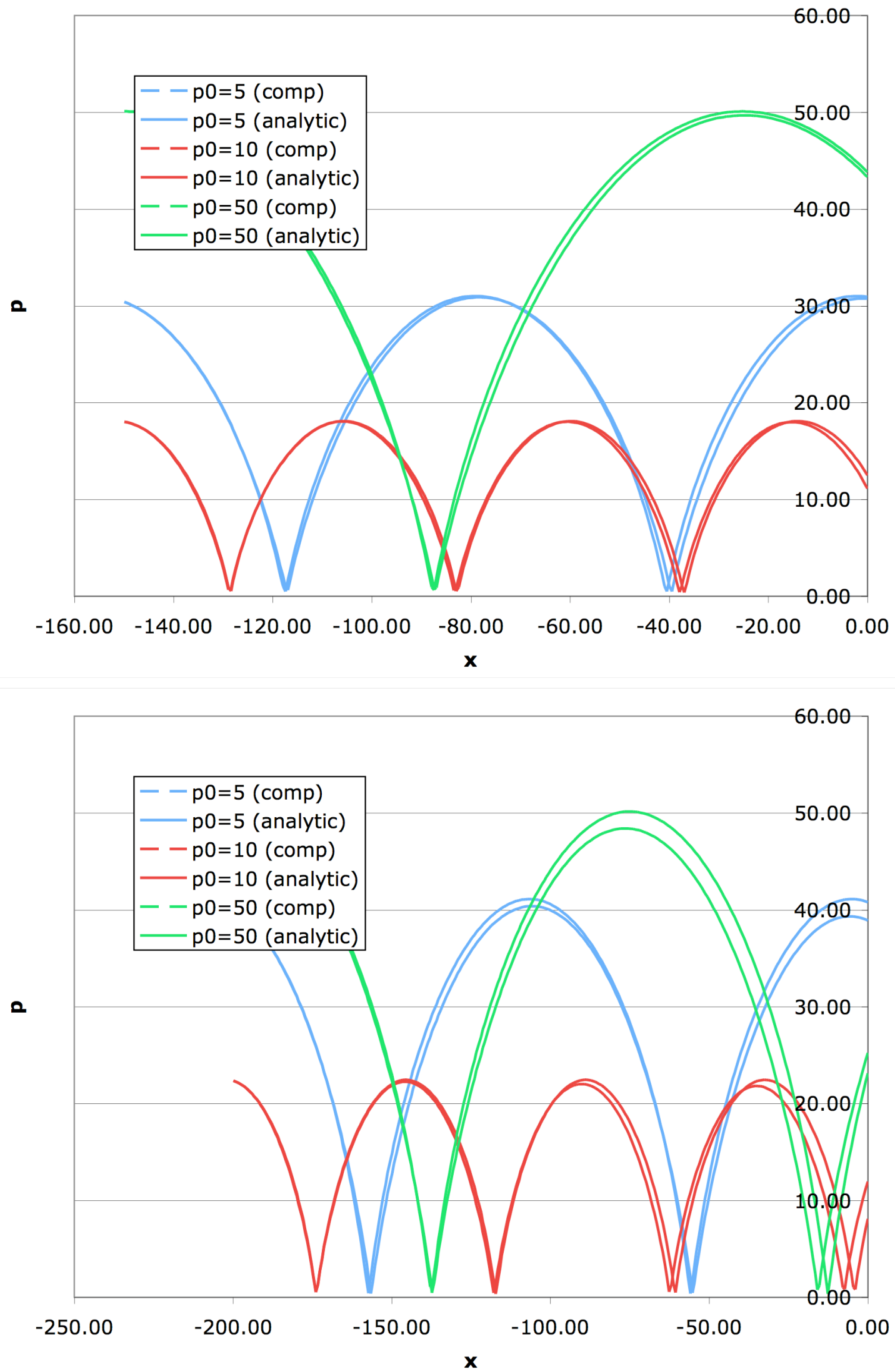


\section{A.5. Listing of the Code}

This section contains a listing of the source code used to generate the data for the plots in the previous section. Not included is the listing of the fourth-order Runge Kutta integrator used for the numerical solutions; it is used via object-oriented library calls. Note that care is taken to handle differences of large numbers for cases where some parameters in the analytic expressions become exponentially large. One can also see the logic used to walk the analytic expressions through as many oscillations of the envelope as needed.

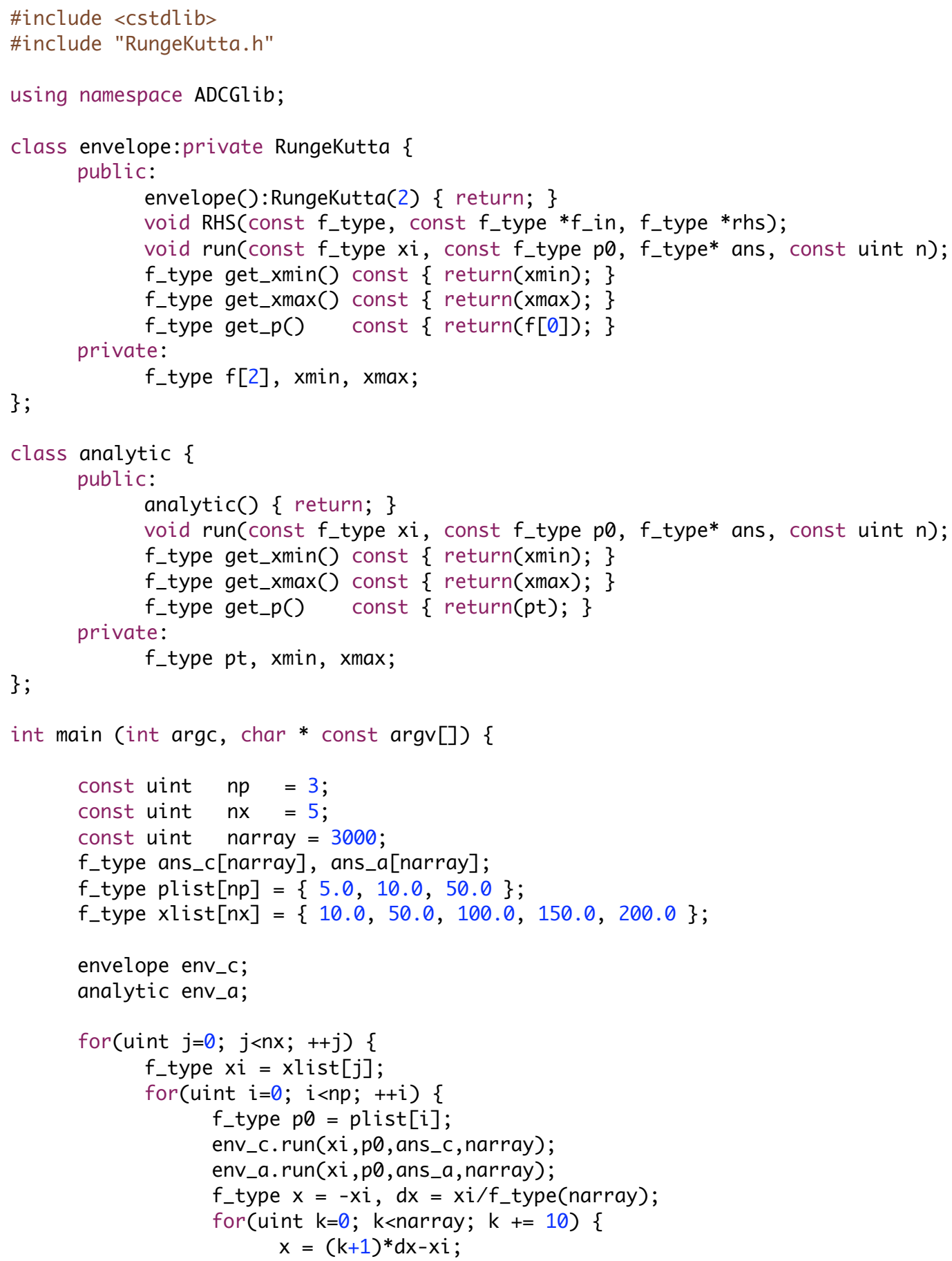




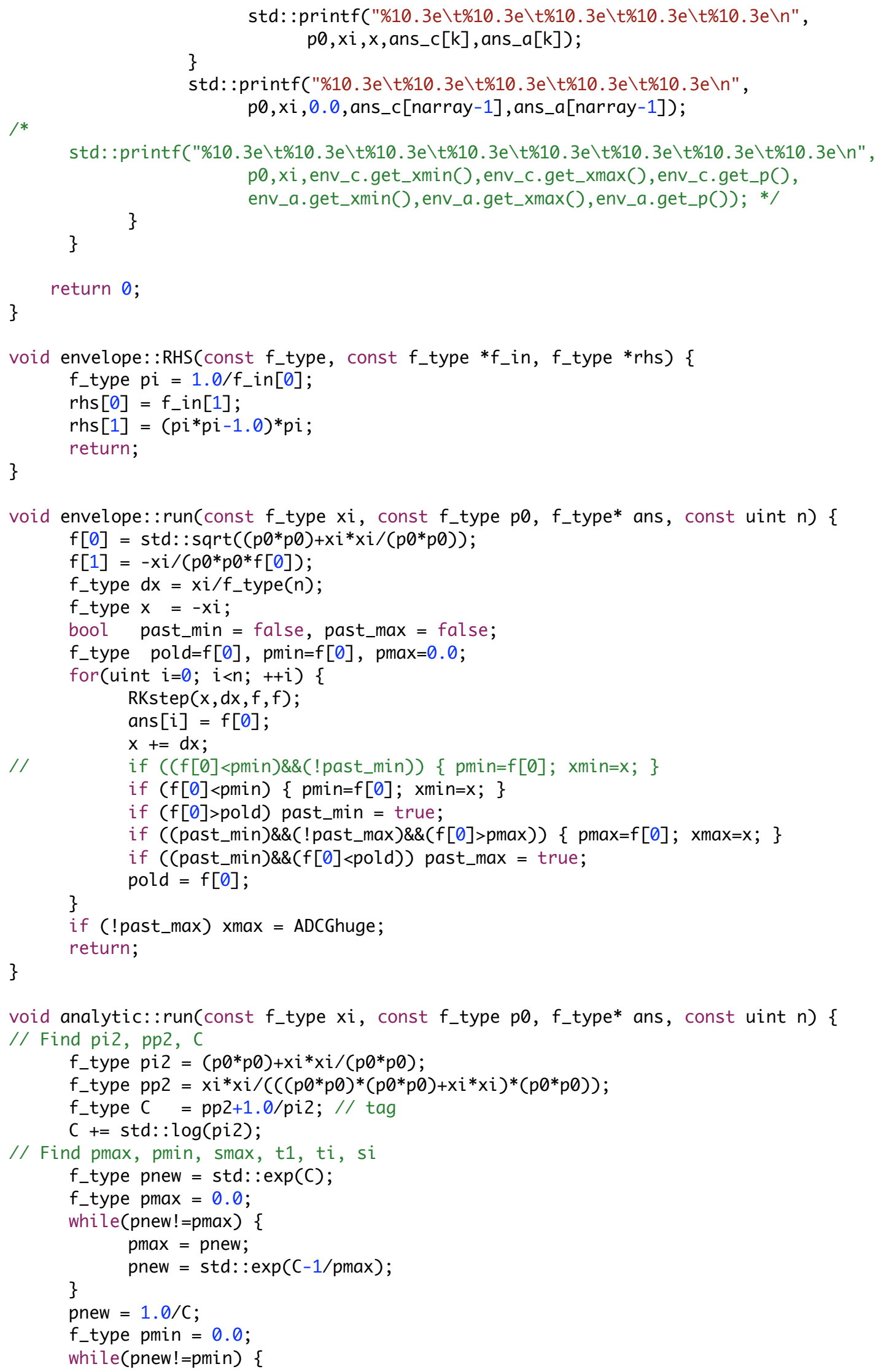




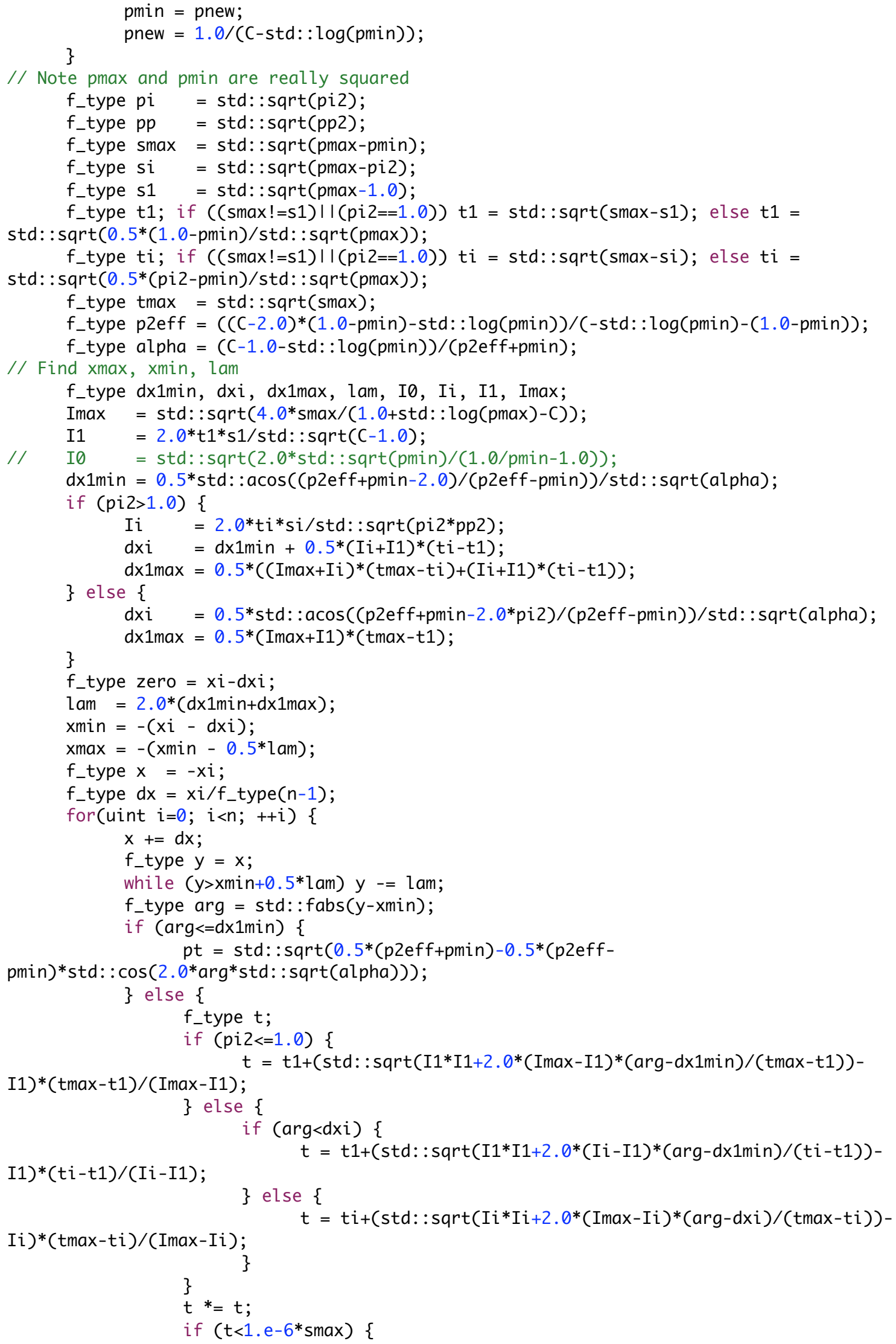




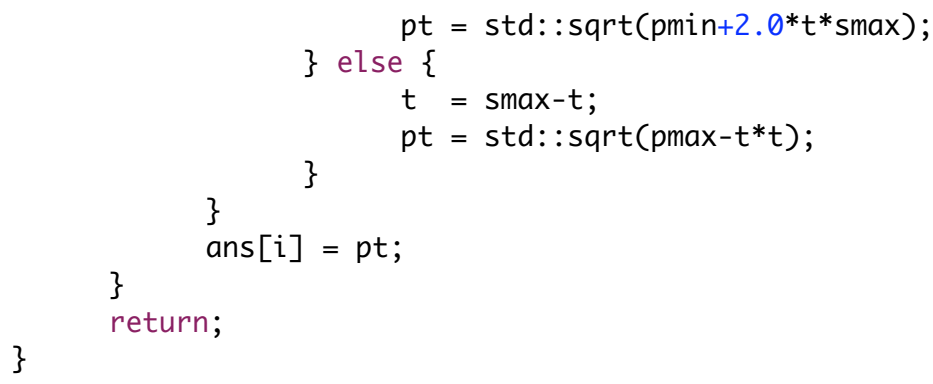

